\title{
Deutsche MedizinischeWochenschrift
}

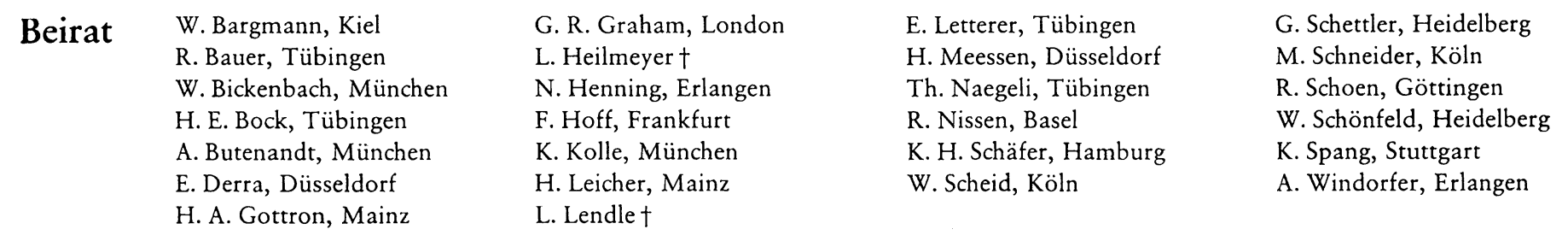

Schriftleitung: Prof. Dr. F. Grosse-Brockhoff Düsseldorf

Prof. Dr. H. Krauss Freiburg i. Br.

Dr. R. H. Rosie stuttgart

Prof. Dr. H. Köbcke†

Inhaltsverzeichnis für den 94. Jahrgang

2. Halbjahr 1969 Hefte 27-52

577 Abbildungen in 767 Einzeldarstellungen und 318 Tabellen

Georg Thieme Verlag · Stuttgart 


\section{Originalarbeiten $^{1}$}

\section{Arzneimittel, Vergiftungen}

\section{Kampfer (T s ch ö p e, E.) 1426}

Cortison-Dauertherapie trotz Zahngranulom? (K a is e r, H.) 1467

Chronischer Gebrauch salinischer Abführmittel (B i e g e r, D.) 1506

Therapeutische Wirkung von Narcotin (I r a v a n i, J.) 1585

Zur esterolytischen Toxogonin-Wirkung (He itmann, R., Felgenhauer, K.) 1583 L. Z.

Einfluß einer Glucocorticoidbehandlung auf die Proteinsynthese in Leberpunktaten von lebergesunden Menschen ( $\mathrm{R}$ i chter, E., Clauditz, S., Leinweber, B.,

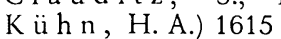

Leukosen unter Tolbutamidbehandlung? (Creutzfeld t, W.) 1625

Diabetes mellitus durch Gonadotropintherapie? (Hohe nf ellner, R.) 1626

Klinik und Therapie der Tetrachlorkohlenstoffvergiftung (D u m e, Th., He r m s, W., Schröder, E., We t z el s, E.) 1646 Benzol-Leukämie ( $\mathrm{H}$ all) 1665 L. Z.

Die Wirkung von Octapressin unter Narkosebedingungen auf Pfortaderdruck, arteriellen Blutdruck und zentralen Venendruck (Henneberg, U., Eckart, J. P., H är ing, R.) 1679

Kontaktallergien durch Nifurprazin (Carofur) (B r a u n, W.) 1685

Nachweiß von Kohlenmonoxid zur Diagnostik der Co-Vergiftung ( $\mathrm{Z}$ or $\mathrm{n}, \mathrm{H}$.) 1692 Benzol-Leukämie ( $\mathrm{H}$ a r t w i c h, G.) 1703

Therapie der Osteoporose mit Natriumfluorid. Morphologische Knochenbefunde bei acht Fällen ( $\mathrm{Kuhlencordt,} F$., Kruse, H.-P., L o z a n o - T o n k in, C., E c k e r me i e r, L.) 1730

Nebenwirkungen der Ovulationshemmer am Auge (Hollwich, F., Verbeck, B.) $1761 \mathrm{~K}$.

Suizid durch Genuß von Bananen (R it t , E., A nd rass y, K., Z i e g l e r, M., Kre mp i e n, B.) 1765 L. Z.

ACTH- oder Glucocorticoid-Therapie? ( $\mathrm{K}$ a i s e r, H.) 1771

Borsäure bei Säuglingen (I p p e n, H.) 1841

Vergleich der enzymatischen Aktivität verschiedener Pankreasfermentpräparate in vitro (M üller - Wieland, K., Berndt, W.) 1870

Beitrag zur Frage der Vitamin-D-Stoßbehandlung. Untersuchungen über den Einfluß des Vitamins D auf den Bindegewebsstoffwechsel und den Mechanismus der ossären Gewebsverkalkung ( $\mathrm{J}$ u n g e - H ü ls i n g, G., Rave, O., W a gner, H., Hüther, W., H ilgen berg, F.) 1877

Xyloseresorption bei chronischem Alkoholismus (B e r n d t, H.) 1893 L. Z.

Odem nach Antiepileptika (S c h u h, F.) 1897

Nitrofurantoin-Fieber (L ü b b e r s, P.) 1922

Hypercalciurie unter PAS-Calcium-Medikation als pathogenetischer Faktor der Urolithiasis. 2. Mitteilung ( $\mathrm{Sch}$ ö n e, D. Hauschild, G., Wässer, St.) 1928

Ursachen medikamentös bedingter parkinsonähnlicher Symptome (J u rn a, I.) 1941 U.
Ist die Verwendung von Oleum camphoratum noch zeitgemäß? ( $\mathrm{B} \mathrm{r} \ddot{\mathrm{u} g ~ m}$ a n n, E.) 1945 L. Z.

Zur Kombinationsbehandlung der akuten Leukämie des Kindes mit Dauno-Rubidomycin ( $\mathrm{S}$ a c h t l e be n, P., B ind e r, H., Krämer, D., Weyrich, C., Schwartz, W.) 1972

Appetitzügler (K u s c h in s k y, G.) 2007

Perkutane Alkoholresorption ( $\mathrm{S} \mathrm{ch} \mathrm{uh}, \mathrm{F}$. 2008

Erythermatodes-Provokation durch Goldtherapie wegen primär-chronischer Polyarthritis (G o e r z, G.) 2040

Kombinationstherapie: Digitalis und Calcium (G r e eff, K.) 2062

Octapressin bei Ósophagusvarizenblutung ( $\mathrm{H}$ a e $\mathrm{m} \mathrm{m}$ e r li, U. P.) 2063

Ist die Furcht vor einer knochenmarkschädigenden Wirkung von Chloramphenicol unbegründet? (Wohlen berg, H.) 2240 L. Z.

Úber die "Rückrechnung " aus BlutalkoholAnalysenwerten bei Trunkenheitsdelikten im Verkehr (Elbel, H., Knüpling, H.) 2259

Gefahren bei der Streptokinase-Behandlung von Herzinfarkten (B a u m a n n, G.) 2296

Suchtgefahr bei Distraneurin ( $\mathrm{H}$ u f $\mathrm{fm}$ a $\mathrm{n}$, G.) 2299

Zur Pathologie der Osteopathie nach Heparinbehandlung ( $S$ chuster, J., MeierRug e, W., Eg li , F.) 2334

Das reversible procainamidinduzierte Lupuserythematodes-Syndrom ( $\mathrm{G}$ ü $\mathrm{n} \mathrm{t}$ h e r, R., A s a m e r, H., D it tri ch, P.) 2338

Digitalisresorption bei Rechtsherzinsuffizien\% (G i $11 \mathrm{~m}$ a n n, H.) 2359

Wurzelreizerscheinungen nach Diuretika? ( J a n z e n, R.) 2408

Zur Therapie der zerebralen Insuffizienz (Grill, P., B r o i c h e r, H.) 2429

Zur Ampicillin-Therapie des Keuchhustens (S i m on, C., B on temps, M., W i e se, K., S c h e w ior-Roland, R.) 2435

Langzeitbehandlung von Immunopathien unter besonderer Berücksichtigung immunsuppressiver Medikamente (S che if f a r t h, F., Warna t z, H.) 2444

Norethisteronönanthat. Untersuchungen während der Anwendung eines langwirkenden parenteralen Kontrazeptivum (G ilf rich, H. J., Nieschlag, E., Dud e c k, J., O ver z i e r, L.) 2473

Klinischer Beitrag zur Frage der optimalen Darreichung von Schilddrüsenhormon (Eickenbusch, W., Haupt, E., L a h r t z, Hg., L e y b old , K.) 2478

Klinisch-experimentelle Untersuchung zur Hemmwirkung verschiedener Corticoide (Hoch he user, W., Gerb, A., Müller-Bardorff, M., Thiele, H.) 2488

Behandlung des multiplen Myeloms mit einem neuen Zytostatikum je-[1-Methyl-5-bis-( $\beta$ chloräthyl)-amino-benzimidazolyl-(2)]buttersäure-hydrochlorid (A n g e r, G., Hesse, P., B a u feld, H.) 2495
Bakteriologische Untersuchungen mit Carbenicillin (B l a s i us, Ch., M e n z, H.-P., S a n d e r, J.) 2501

Zum gegenwärtigen Stand der Lithium-Therapie (L a u t e r, H.) 2512 U.

Einfluß oraler Ovulationshemmer auf klinischchemische Normalwerte (W il b e r t, L.) $2522 \mathrm{~K}$.

Nitrofurantoin-Fieber ( $\mathrm{L}$ a $\mathrm{u}$ b e $\mathrm{n} \mathrm{t} \mathrm{h}$ a $1, \mathrm{~F}$.) 2523 L. Z.

Behandlung mit $\beta$-Rezeptorenblockern (L y dt i n, H.) $2560 \mathrm{~A}$. Th.

Carbenoxolon-Natrium (O t t e $\mathrm{n}$ j a n n, R.) 2566

Schäden bei immunsuppressiver Therapie (W i o n tze k, H.) (Kölle, G.) 2560 L. Z.

Nebenwirkungen von Diazepam (Kuschinsk y, G.) 2571

Erfahrungen mit »ultrahohen" Cyclophosphamid-Dosen an 42 Kranken mit soliden und lymphoretikulären Tumoren $(\mathrm{S} \mathrm{Ch} \mathrm{u}$ bert, J. C. F., Fis cher, M., Hil bert, L.) 2583

Indikationen für halbsynthetische Penicilline (R e b e r, H.) 2607 A. Th.

Vasopressin und Oxytocin ( $\mathrm{R} \mathrm{a} \mathrm{u} \mathrm{ch,} \mathrm{R.)}$ 2611 U.

Beitrag zum renalen Kaliumverlustsyndrom. Bericht über einen Fall von geleugnetem Diuretika-Abusus (A dolphs, H.-D. I r m s cher, K., Solb a ch, H. G.) 2634

Die Wirkung von Cumarin, Indandion- und Benzofuran-Derivaten auf die renale Harnsäureausscheidung (Z öllner, N., Gröbner. W.) $2652 \mathrm{~K}$

Oher die Beeinflussung von Herzrhythmusstörungen durch $\alpha$-Propranolol (A m or H., Dienstl, F., Judmaier, G., Schwingshackl, H.) 2669

Behandlung des Morbus Parkinson mit 1.-Dopa in Kombination mit einem Decarboxylosehemmer (S i e g f ri ed, J., $\mathrm{Kl}$ a iber, R., Perret, E., Zieg ler, W. H.) 2678

Proteinurie bei Glibenclamid-Behandlung? (K ü hn a u, J.) 2703

\section{Atmungsorgane}

\section{eirischließlich Lungentuberkulose}

Hypercalciurie unter PAS-Calcium-Medikation als pathogenetischer Faktor der Urolithiasis. 1. Mitteilung ( $\mathrm{S} \mathrm{ch}$ ö n e, D., $\mathrm{H}$ a u s child, G., W äs s e r, S t.) 1491

Behandlungsergebnisse bei zufällig entdecktem und symptomlosem Bronchialkarzinom (B e r nd t, H.) 1559

Die Strahlenbehandlung des Bronchialkarzinoms (Vi e t e n, H., G ün the r, D.) 1593

Veränderungen des Bronchialwiderstandes bei Asthmatikern nach Applikation von Pilocarpin in den Konjunktivalsack (B r u ch hausen, D., Haschem, J., Dar d e nn e r, M. U.) 1651

Die Bedeutung der Pleura- und Lungenbiopsie in der internen Diagnostik ( $\mathrm{M} \ddot{\mathrm{u}} \mathrm{rtz}, \mathrm{R}$., B ege nat, H.) 1724 
Neue Normalwerte für die Lungenfunktionsprüfung mit der Ganzkörperplethysmographie (Amrein, R., Keller, R., Joos, H., H e r z og, H.) 1785

Klinische Differenzierung des asthmatischen Syndroms (W e r n e r, M.) 1802

Erfolgreiche immunsuppressive Behandlung eines Kranken mit essentiellen Lungenhämosiderose ( $\mathrm{Schubert}$, J. C. F. $\mathrm{Schubert}, \mathrm{H} ., \mathrm{Ne} u, O)$.

Das Goodpasture-Syndrom. Ein klinischer und pathologisch-anatomischer Beitrag (Krauss, J., Red enbacher, M., Streicher, E.) 1820

Transpulmonale arterio-venöse Fistel zwischen Körper- und Lungenkreislauf (v. E g i d y, H., H a b i g h o r s t, L. V., T a l ke, H.) 1917

Angeborene pulmonale Lymphangiektasien. Bericht über 4 Fälle ( $\mathrm{Gl}$ e is s ner, $\mathrm{P}$., A s an te, F., Schubert, G. E.) 1987

Therapie der Lungenmykosen (W e $\mathrm{g} m$ a $\mathrm{n} n$, F.) $1992 \mathrm{~A}$. Th.

Das Lungenmycetom und seine Differentialdiagnose ( $\mathrm{R}$ e in h a r d t , K.) 2045

Pneumocystis-carinii-Pneumonie beim $\mathrm{Er}$ wachsenen ( $F$ i s c h e r, R., B e c k e r, H. D., J o is t, J. H., T is m e r, R.) 2135

Erhöht vorsorgliche Röntgenreihenuntersuchung die Uberlebensrate beim Lungenkrebs? (O e s e r, H.) (B e r n d t , H.) 2180 L. Z

Lungenembolie und symptomatische Psychose (R i e d e r e r, J.) 2181 L. Z

Röntgenologische Lungenbefunde bei septischen Erkrankungen (D u n k e r, S., Stille, W.) 2026

Therapie der chronischen Bronchitis $(\mathrm{G}$ a $\mathrm{rt}$ m a n n, J.) 2234 A. Th.

Ätiologie und Diagnostik der Lungenfibrose (U r th a le r, F.) 2290 A. D.

Die chronisch obstruktiven Atemwegserkrankungen des Bergmanns (R e i chel, G., Ulm e r, W. T., B u ck u p, H., S te mpel, G., Werner, U.) 2375

Spontanpneumothorax (M a r x, H. H.) 2408

Gutartige Verlaufsform einer pulmonalen alveolären Proteinose (W e l s c h, A.) 2438

Zur Antibiotikatherapie der Nocardiose (S c h a a l, K. P., L e i s c h i c k, W.) 2505

Mykogene Allergie. Schimmelpilz- und Pilzsporen als Ursache allergischer Atemwegsleiden (V i r c h ow, C h r.) 2508 A. D.

Möglichkeiten der spezifischen Grippeprophylaxe (G e r t h, H.-J.) 2510

Das IgA- und IgG-System bei spastischer Bronchitis im Kindesalter ( $T$ ü $\mathrm{rk}, \mathrm{E}$., W i ersbitzky, S.) 2554

Tuberkulinprobe in der Differentialdiagnostik (H e s s, W.) 2568 L. Z.

Diagnostische Maßnahmen bei Verdacht auf Lungeninfarkt (M o r a w e t $\mathrm{z}$, F.) 2605 A. D.

Hühnerzüchterlunge (B ü t i k of e r, E., d e W e c k, A. L.) 2627

Uber eine Familie mit Goodpasture-Syndrom, Alport-Syndrom und Asthma bronchiale (M üntefering, H., Hils cher, W., $\mathrm{M}$ i e s e, A.) 2637

Lungenfunktionsprüfung in der Praxis (Reich el) 2703

\section{Augen}

Sichtverbesserung durch gelbe Blendschutzbrille? ( $\mathrm{S} \mathrm{ch}$ o b e r, H.) 1545

Katarakt durch Röntgenstrahlen (K r o k ow s k i, E.) 1585
Veränderungen des Bronchialwiderstandes bei Asthmatikern nach Applikation von Pilocarpin in den Konjunktivalsack (B r u chh a usen, D., H a schem, J. D a rde n ne, M. U.) 1651

Nierendurchblutung und Glomerulumfiltrat unter Dihydralazin-Wirkung (W ü $\mathrm{r} \mathrm{d}$ i n ger, H., A ust, W., M ölle r, U.) 1688

Nebenwirkungen der Ovulationshemmer am Auge (Hollw ich, F., Verbeck,B.) 1761

Elektrophoretische und immunologische $\mathrm{Be}$ funde beim Marfan-Syndrom ( $\mathrm{S}$ u s $\mathrm{c}$ h k e J., Heilmann, K., Murken, J.-D., St e hr, K.) 2289

Art und Häufigkeit der Augenbeteiligung nach parenteraler Goldtherapie ( $\mathrm{R}$ o d e n h ä us e r, J. H., B e h r e n d, T.) 2389

\section{Bewegungs- und Stützapparat}

Therapie des Sudeck-Syndroms (C o t t a , H.) 1505

Röntgentherapie des Morbus Bechterew (D i h l m a n n, W.) 1505

Die Behandlung des Periarthritis-humeroscapularis-Syndroms (W a g e n h ä u s e r, F. J.) $1579 \mathrm{~A}$. Th.

Úberlastung nach Oberschenkelamputation (G r o h, H.) 1626

Wirbelsäulen- und Gelenkschäden nach Beinamputation (K o c h, F.) 1706

Therapie der Osteoporose mit Natriumfluorid. Morphologische Knochenbefunde bei acht Fällen (Kuhlencordt, F., Kruse, H.-P., L o z a n o-T on kin, C., E ckerme i e r, L.) 1730

Vererbbarkeitsrisiko des angeborenen Klumpfußes ( $\mathrm{M} \mathrm{a} \mathrm{u}, \mathrm{H}$.) 1950

Quantitative Mineralsalzbestimmung am kindlichen Skelett (Schuster, W., Re is s, K. H., K r a m e r, K.) 1983

Therapie bei rheumatischem Fieber ( $\mathrm{S}$ ü $\mathrm{d}$ h of , H.) 2007

Rezidivierende Polychondritis mit Aortenaneurysmen. Jaksch-Wartenhorst-v. Meyenburg-Altherr-Uehlinger-Syndrom ( $\mathrm{L}$ a n $\mathrm{g}$, H. D., M ü ll e r, D., Fi n k e, J.) 2033

Erythematodes-Provokation durch Goldtherapie wegen primär-chronischer Polyarthritis (G o e r z , G.) 2040

Polymyalgia rheumatica ( $\mathrm{K}$ a i s e r , H.) 2232 A. D.

Knochenmarksschädigung bei immunsuppressiver Therapie der juvenilen rheumatoiden Arthritis und des Still-Syndroms mit Azathioprin (K öll e, G.) 2268

Zur Pathologie der Osteopathie nach Heparinbehandlung (S chuster, J., MeierRuge, W., Eg li , F.) 2334

Verhütung von Skelettfehlbildungen nach mütterlichem Blutverlust durch Elektrolytersatz (Grote, W.) 2342

Redondrainage nach Osteotomien $(\mathrm{S} \mathrm{chle}$ g e 1, K. F.) 2359

Art und Häufigkeit der Augenbeteiligung nach parenteraler Goldtherapie ( $\mathrm{R}$ o d e n h ä u s e r, J. H., B e r e n d, T.) 2389

Die Vor- und Nachteile der Bauchlage bei Neugeborenen und jungen Säuglingen G le is s, J.) 2449 K.

Thorium-X-Behandlung beim Morbus Bechterew ( $\mathrm{H}$ a i k e , H. J.) 2459

Schäden bei immunsupressiver Therapie (W i o n tzek, H., Kölle, G.) 2568 L. Z.

Die Beteiligung innerer Organe bei der Polyarthritis chronica progressiva (B a i e $\mathrm{r}, \mathrm{M}$.) 2644 A. D.

\section{Biographien und Nachrufe}

Hans Helmut Wolter 1925-1969 (S c he t t le r, G.) 1464

Max Gänsslen 1895-1969 (M a rt ir., H.) 1583

40 Jahre Herzkatheterismus ( $\mathrm{Sch}$ a d e w a ld t , H.) 1769

Prof. Dr. med. Dr. med. h. c. Herwig Hamperl zum 70. Geburtstag (G e d ig k 1894

Die Poliklinik. Ihre Funktion u. ihr Aufbau in der modernen Medizin ( $\mathrm{Z}$ ö $11 \mathrm{n}$ e r, W.) 1933

Die Herztransplantation des Höllensekretärs Lu. Ein chinesisches Märchenmotiv (S ch r a m m, G.) 1945

Zur Entdeckung der DNS durch Friedrich Miescher vor 100 Jahren ( $\mathrm{H}$ a r be r s, E.) 1948

Erinnerung an Hans Berger ( $\mathrm{K} \mathrm{l}$ a p e t e $\mathrm{k}, \mathrm{J}$.) 2123

Das Horoskop des Dr. med. Leonhart Rauwolff (P ü s c h e l, E.) 2245

Erste Nephrektomie vor 100 Jahren: Gustav Simon (1824-1876) (K e ll e r , J.) 2247

Nobelpreis für Medizin und Physiologie 1969 (S t a r ling e r, P.) 2405

Otmar Freiherr von Verschuer $(\mathrm{S} \mathrm{ch}$ a d e, H.) 2407

Ludwig Lendle + 19.8.1969 (E rdman n, W. D., Friedberg, Kl. D., Hasselblatt, A., S c h m id t, G.) 2455

Achtzig Jahre Uncinatus-Anfälle (W o r m s e r, P.) 2618

Ludwig Heilmeyer in memoriam. Improvisationen anläßlich der akademischen Trauerfeier am 10. September 1969 (W e is b e k k e r, L.) 2657

Heiz Koebcke (1895-1969 (K a t z , P. F.) 2701

\section{Blut und blutbildende Organe}

Aseptische Wunddehiszenzen und Gerinnungsfaktoren (B o r m, D., K a is e r, N.) 1401 Echinacin-Test zur Leukozytenprovokation bei effektiver Strahlentherapie ( $\mathrm{C}$ h o n é, B., $\mathrm{M}$ a n id a k is, G.) 1406

Mikrochemische Urineisenbestimmung bei Säuglingen und Kindern. Untersuchungen zur Urineisenbestimmung vor und nach Deferoxamin-Gabe bei Gesunden, Patienten mit Eisenmangel und Eisenüberladung (M a a s, B., N e th, R., Hoschenhofer, D. Schäf er, K. H.) 1410

Antikoagulantientherapie bei Mitralstenose (S c h m u tzle r, R.) 1424

Leukämie durch Röntgenstrahlen (Fli e d n e r, T. M., A d a m, W.) 1426

Eisenresorption bei Achlorhydrie ( $\mathrm{M}$ o n $\mathrm{tz}$, R.) 1503 L.Z

Leukosen unter Tolbutamidbehandlung: (Creutzfeldt, W.) 1625

Benzol-Leukämie ( $\mathrm{H}$ a 11 ) 1665 L. Z.

Nachweis von Kohlenmonoxid zur Diagnostik der CO-Vergiftung ( $\mathrm{Z}$ or $\mathrm{n}, \mathrm{H}$.) 1692

Benzol-Leukämie (H a r tw i c h , G.) 1703

Ein methodischer Beitrag zur Funktionsprüfung menschlicher Blutplättchen unter standardisierten Bedingungen ( $\mathrm{G}$ ross, R., $\mathrm{N}$ i e me y e r, G., R e u ter, H.) 1735

Colonresektion bei schwerer Hämophilie $\mathrm{A}$ unter dem Schutz von antihämophilen Kryopräzipitaten (B r ü s t e r, H., R i n g le r, W., Glas sner, K., Ri e ch, P.Chr., $\mathrm{H}$ i m melb a ch, E.) 1799

Erfolgreiche immunsuppressive Behandlung eines Kranken mit essentieller Lungenhämosiderose ( $\mathrm{Schubert}$, J. C. F. S chubert, H., $\mathrm{Ne} \mathrm{u}, \mathrm{O}$.) 1813 
Verzögerte Reaktion von Morbus HodgkinLymphozyten auf Phytohämagglutinin ( $\mathrm{H}$ a v e m a n n, K.) 1867

Therapie der Hämophilie ( $\mathrm{t} r \mathrm{a} u \mathrm{~b}, \mathrm{P}$. W.) $1885 \mathrm{~A}$. Th.

Die paroxysmale nächtliche Hämoglobinurie eine »myeloproliferative Erkrankung «? (M a r t in, H.) $1891 \mathrm{~K}$

Zur Kombinationsbehandlung der akuten Leukämie des Kindes mit Dauno-Rubidomycin (S a chtleben, P., Binder, H., Krämer, D., Weyrich, C., Schwartz, W.) 1972

Zur Induktions- und Intervall-Behandlung der Leukämien von Kindern und Jugendlichen (Oehme, J., Meyer-Beeck, D.) 1993 U.

Thiamin-bedürftige megaloblastische Anämie ( $\mathrm{N}$ i e d e r h off, H., K ün z e r, W.) 2000 $\mathrm{K}$.

Malabsorptionssyndrom bei partiellem Immunglobulinmangel (JgA-Defekt) (W a g n e r, A., Gros s m a n n, H. D.) 2023

Kälteurtikaria und Kininsystem (D u c k, H.J.) 2059 L. Z.

Erfolgreiche Operation eines Pseudotumors nach Oberschenkelfraktur bei Hämophilie B. Zugleich ein Beitrag zur Plasmaphorese (F is che r, M., Fuchsig, P., Le chner, K., Pilgerstorfer, H. W., S alzer, Mechthild, Steinbereith. n e r, K.) 2145

Myelofibrose und Osophagusvarizen (W o h le n b e r g, H.) 2163

Granulozytopenie (G e h r m a n n, G.) 2182

Die derzeitige Häufigkeit der einzelnen Lymphknotenerkrankungen in SchleswigHolstein (L e n n e r t, K.) 2194

Ist die Furcht vor einer knochenmarkschädigenden Wirkung von Chloramphenicol unbegründet? (W o h l e n b e rg, H.) 2240 L. Z.

Polyzythämiker als Blutspender? (S p i e I$\mathrm{m}$ a $\mathrm{n} \mathrm{n}$, W.) 2250

Knochenmarksschädigung bei immunsuppressiver Therapie der juvenilen rheumatoiden Arthritis und des Still-Syndroms mit Azathioprin (K ölle , G.) 2268

Pseudo-Pelger-Zellen vom homozygoten Typ bei unreifzelliger myeloischer Leukämie (H e n n e ke us e r, H.-H., F i s c h e r, R., T a l ke, H., M a in ze r, K.) 2284

Thrombolysetherapie (S c h i m p f, Kl.) 2292

Zytochemische Differenzierung und Krankheitsverlauf akuter Leukämien des Kindesalters (B e ckm a n n, H., N e th, R. Landbeck, G., Ma as, B., Schmidtke, H.) 2320

Moschcowitz-Syndrom. Ein Beitrag zur »thrombotisch thrombozytopenischen Purpura " mit gerinnungsphysiologischen und pathologisch-anatomischen Untersuchungen (L e chler, E., Klein, H. O., Schu m a cher, K., Gross, R., Ed er, M. K u n s t, H.) 2326

Unspezifische erworbene Makrophagenresistenz (G ün th e r, O.) $2355 \mathrm{~K}$.

Perniziosa-Therapie (Pribilla, W.) 2356 Das Di-Gugliemo-Syndrom im Kindesalter (D rescher, J., H ansen, H. G., A lt hoff, H., Graucob, E.) 2415

Zur Problematik der akuten Erythroblastose des Erwachsenen (Morbus Di Guglielmo) (v. W a e ch t e r, R., M e y e r - B u r g, J., $\mathrm{Ne} \mathrm{u} \mathrm{b}$ a u e r, F. W.) 2421

Langzeitbehandlung von Immunopathien unter besonderer Berücksichtigung immunsuppressiver Medikamente (S che if farth, F., Warnatz, H.) 2444 U.

Thrombotest und Quick-Test (Vos s, D.) 2525
Neue Behandlungswege bei großen chirurgischen Eingriffen an Patienten mit Hämophilie A (Wedell, J., R ingler, W., B r üs t e r, H., R i e c h, P.-Chr., G ö b el, U., Pfizner, W.) 2539

Zellmorphologie und Prognose der Paraleukoblastenleukämie ( $\mathrm{M}$ u n d, J., J a c o b i , H., K ün z e r, W.) 2550

Laboratoriumsbefunde bei Paraproteinämien (K l e m m, D.) 2558 A. D.

Schäden bei immunsuppressiver Therapie (W i o n t z e k, H., Köll e, G.) 2568 L. Z.

Leukämie nach Extremitätenamputation? (O b r echt, P.) 2571

\section{Chirurgie}

Aseptische Wunddehiszenzen und Gerinnungsfaktoren ( $\mathrm{B}$ or $\mathrm{m}, \mathrm{D}$., $\mathrm{K}$ a i s e $\mathrm{r}, \mathrm{N}$.) 1401

Interne Liquordrainage bei Hirntumoren mit Hydrozephalus (D r i e s e n, W., T od orow, S.) 1418 A. Th.

Kombinierte spezifische Immunsuppression (G ü n th e r, O.) $1421 \mathrm{~K}$.

Die akute Osophagusvarizenblutung. Ein Aufruf zu aktiver Therapie (Esser, G. $\mathrm{G}$ ü $\mathrm{tg}$ e $\mathrm{m}$ a n n, A.) 1476

Elektronische Verbesserung von Röntgenaufnahmen (R o th, F.- J., W e n $\mathrm{z}$, W., K r a m e r, H.) 1483

Arteriomesenteriale Duodenalkompression (H a f t e r, E.) 1495 A. D.

Die Bedeutung der Mikrochirurgie in der Hirnchirurgie (Y a s a r g i l, M. G.) 1496 A. Th.

Kryochirurgische Behandlungsverfahren in der Urologie (O p e lt , B., S ö k e land, J., Z e m a n, N.) 1497 U.

Therapie des Sudeck-Syndroms (C o t t a , H.) 1505

Tonus und Motilität von Magen und Zwölffingerdarm nach selektiver Vagotomie und Pyloroplastik (D a h m, K., S a p o u n o v, S.) 1513

Ergebnisse der direkten und indirekten Isthmusplastik bei Kindern und Jugendlichen unter besonderer Berücksichtigung des Anastomosenwachstums (H e h r le in, F. W.) 1522

Die Diagnostik der muskulären subvalvolären Aortenstenose (W e n g e r, R.) 1537 A. D

Die Therapie der muskulären subvalvulären Aortenstenose (W e n g e r, R.) 1539 A. Th.

Die Behandlung des Periarthritis-humeroscapularis-Syndroms (W a g e $\mathrm{n}$ h ä u s e $\mathrm{r}$, F. J.) 1579 A. Th.

Schmerzen bei Amputationsneurom ( $\mathrm{M} \mathrm{u}$ $\mathrm{m}$ e $\mathrm{n} \mathrm{th}$ a le r, M.) 1585

Der Hydrozephalus des Erwachsenen ( $\mathrm{B} \mathrm{e}$ n i n i, A.) 1617 A. D.

Die elektromagnetische Registrierung von Stromzeitvolumina (M e issne r, $H$. Messmer, K.) 1621 U.

Úberlastung nach Oberschenkelamputation (G roh, H.) 1626

Die Wirkung von Octapressin unter Narkosebedingungen auf Pfortaderdruck, arteriellen Blutdruck und zentralen Venendruck (Henneberg, U., Eckart, J. P. H är in g, R.) 1679

Kontaktallergien durch Nifurprazin (Carofur) (B r a u n,W.) 1685

Wirbelsäulen- und Gelenkschäden nach Beinamputation (K o c h, F.) 1707

Erfahrungsbericht über eine homologe Lebertransplantation (G ü t g e m a n n, A., Schriefers, K. H., Esser, G., Lee T. S., P a qu e t, K. J., Kä u f e r, C.) 1713
Die Behandlung spontaner Hypoglykämien bei einem inoperablen Leberzellkarzinom durch Glucagon ( $M$ üh e, E., S chrik k e r, K. T., R a it h e l, D.) 1781

Anatomische und technische Möglichkeiten der intravenösen Infusionsbehandlung (S c hult e, H. D.) 1793

Colonresektion bei schwerer Hämophilie A unter dem Schutz von antihämophilen Kryopräzipitaten (B r ü s t e r, H., R in g ler, W., Glassner, K., Riech, P.-Ch r., Hi m m elb a ch, E.) 1799

Infektionen mit Pasteurella multocida nach Tierbissen (Stille, W., Stoll, L., $\mathrm{Hel} \mathrm{m}$, E.) 1816

Hepatozellulärer Ikterus nach wiederholter intravenöser Infusion von Fettemulsionen bei Kindern mit ausgedehnter Darmresektion (Olbing, H., Engels, A.-M., D öng e s, K. G.) 1825

Die Revaskularisation des Myokards bei koronarer Herzkrankheit ( $\mathrm{H} \mathrm{e} \mathrm{g} \mathrm{e} \mathrm{m} \mathrm{a} \mathrm{n} \mathrm{n}$, G., B a ch m a n n, K., D it t r i ch, H.) 1903

Die Diagnose des akuten Mesenterialgefäßverschlusses ( $\mathrm{H} \mathrm{u} \mathrm{b} \mathrm{e} \mathrm{r,} \mathrm{F.} \mathrm{B.)} 1937 \mathrm{~A}$. D.

Pharmakoangiographie des Pankreas mit Sekretin und Adrenalin (C e n, M., R o senbusch, G., Frick, W., Kalff, G.) 1970

Nochmals: Arzt und Transfusionsverweigerer (Koh l h a a s, M.) 2005

Die Bedeutung der chirurgischen Vor- und Nachbehandlung bei Organtransplantationen (P i c hl m a i e r, H., Edel, H. H., L a ve nd e r, R.) 2082

Zur Klinik der Dünndarmdivertikel (W a g n e r, A.) 2097

Intermittierender Verschluß der Arteria poplitea durch zystische Adventitiadegeneration (E h r in ge r, H., D e n c k, H., W u k e t i ch, S t., B run ne r, E.) 2107

Indikationen zur direkten Cholezysto-Cholangiographie und Cholangiographie (W a n n a g a t, L.) 2111 A. D.

Die künstliche Harnableitung ( $\mathrm{M}$ a y, P., Sökel and, J.) 2115 Ư.

Zur Problematik des dissoziierten Hirntodes bei Patienten eines internistischen Intensivpflegezentrums (Schuster, $H$. P., Busch, H., Busch, G., Wi em c zyk, H., B a um, P., Knolle, J., Frh. v. Ungern-Sternberg, A., $\mathrm{L}$ a n g, K.) $2118 \mathrm{~K}$.

Korrelation des laparoskopischen und histologischen Befundes bei chronischer Hepatitis und Leberzirrhose (Solte r m a n n, W.) 2121 L. Z

Arzt und Operationsverweigerer (Kohl$\mathrm{h}$ a a s, M.) 2122

Erfolgreiche Operation eines Pseudotumors nach Oberschenkelfraktur bei Hämophilie B. Zugleich ein Beitrag zur Plasmapherese (F is che r, M., Fuchsig, P., Lechner, K., Pilgerstorfer, H. W., Salzer, Mechthild, Steinber e it h n e r, K.) 2145

Leberzirrhose nach Magenresektionen, bei Magen-Duodenalgeschwüren und $\mathrm{Ge}$ schwürsnarben (M ö r l, H., F e i g e, G.) 2167

Zum Problem der sogenannten Transplantationslunge (B e c k e r, H. D., $\mathrm{H} \mathrm{ü} \mathrm{b} \mathrm{n} \mathrm{e} \mathrm{r,}$ G., Eigle r, F. W., S i eb e rt, H. G.) 2202

Schwere traumatische Hämolyse und Endokarditis bei Faszienklappenplastik der Aorta. Korrektur durch Kugelklappe (S t r a u b, P. W., Rothlin ; M.) 2212 
Bauchdeckenerschlaffung. Früh- und Schweresymptom bei Magenblutung ( $\mathrm{B}$ i e r s t e d t P.) $2241 \mathrm{Ch}$.

Polyzythämiker als Blutspender? (S p i e lm a n n, W.) 2250

Der Wert des submaximalen Betazoltestes für die Indikation bionomer nichtresezierender Operationsverfahren beim Magen- und Zwölffingerdarmgeschwür ( $\mathrm{R}$ e h n e r, $M$., K o c h, W.) 2278

Thrombolysetherapie (S c h i m p f , K l.) 2292 A. Th.

Die chirurgische Behandlung des akuten Arterienverschlusses (Voll $\mathrm{m}$ a r, J., L a u b a c h, K., Gru s s, J.D.) 2315

Phantome nach Amputation und bei angeborenem Gliedmaßenmangel ( $\mathrm{P}$ o e c k, K.) 2367

Behandlung des "akuten " Harnsteines ( $\mathrm{V}$ a h le n s i e ck, W.) 2381

Indikationen, Möglichkeiten und Grenzen der selektiven Oberbaucharteriographie ( $\mathrm{P} \mathrm{r} \mathrm{e}$ t e r, B., W i r th, W.) 2395 A. D.

Zur Feststellung des Hirntodes $(\mathrm{S} \mathrm{ch}$ ne id e r, H.) 2405 L. Z.

Allgemeine Grundlagen der Diagnostik von Magen-Frühkarzinomen mit der Gastrokamera (O s h i m a, H.) 2440 A. D.

Behandlungsmöglichkeiten bei Hypophysentumoren (W e be r, G.) 2442 A. Th.

Neue Behandlungswege bei großen chirurgischen Eingriffen an Patienten mit Hämophilie A (Wedell, J., R ing le r, W., B rüster, H., R i e ch, P.-Chr., Göb e 1, U., Pfizner, W.) 2539

Klinik und Therapie des Carpaltunnelsyndroms (W e s s in $\mathrm{g} \mathrm{h}$ a g e, D.) 2544

Leukämie nach Extremitätenamputation? (O b r e ch t, P.) 2571

Chirurgische Resultate bei muskulärer Subaortenstenose (R o thlin, M., Wirz, P., Preter, B., Gat tiker, H., Senn in $g$, A.) 2579

Primärer Hyperparathyreoidismus infolge multipler, eutopisch und dystopisch gelegener Epithelkörperchenadenome (D i e t he $\mathrm{lm}$, L., Gerok, W., K ü m m e r le, F., Pabst, K., Hennekeuser, M. H.) 2593

Der Wilms-Tumor im ersten Lebensjahr. Insbesondere über 62 Nephroblastome des Neugeborenen (B a ch mann, K. D., K r ö l1, W.) 2598

Der Vena-subclavia Katheter (Volles, E., Gressner, P., Dahlmann, W., Prill, A., S a b u n c u, N.) 2682

Diagnose der Fettembolie (P e l z l, H.) 2690 A. D.

\section{Ernährung, Diätetik}

Xyloseresorption in Beziehung zu Leberfunktion und Ernährung bei chronischem Alkoholismus (I r sigle r, K., P o in te r, H., Krys pin-Exner, K.) 1532

Hafertage beim Diabetes ( $\mathrm{S}$ a u e r, H.) 1771

Diätetische Therapie bei Niereninsuffizienz (Kluth e, R., Quirin, H.) 2112 A. Th.

Diättherapie der essentiellen Hyperlipämien (Gries, F. A., Jahnke, K., Pre is s, H., C a nzle r, H., M is s, H. D.) 2307

Pollenallergie durch Honig ( $\mathrm{K}$ üs te r, F.) 2458

Nahrungsbedarf diabetischer Kinder

(S a chne, R., S a chne, B., J a hn ke, K., D a w e ke, H.) 2535

Karzinogene Wirkung von Cyclamat? (Menden, E.) 2619

\section{Geburtshilfe und Frauenheilkunde}

Zur Frage der Anti-Rh $(D)$-Prophylaxe in der Schwangerschaft ( $\mathrm{H}$ a e r in $\mathrm{g}$, M.) 1433

Zur Wirkung von Immunglobulin-Anti-D im Rh-positiven Organismus. Úbersicht über bisher beobachtete Fehlanwendungen des Immunglobulins-anti-D ( $\mathrm{N}$ i e de $\mathrm{r} h$ of $f$, N., Schneider, J., Stachow, P., K ünzer, W.) 1437

Bakteriurien in der Schwangerschaft. Epidemiologie, geburtshilfliche Komplikationen und Behandlungsergebnisse $(\mathrm{H}$ i r s ch, H. A., D e c k e r, R.) 1553

Perinatale Mortalität von Kindern mit Distress-Hinweisen vor dem Einsatz neuzeitlicher geburtshilflicher Uberwachungsverfahren ( $\mathrm{P} \mathrm{a} \mathrm{k} \mathrm{z} \mathrm{a} \mathrm{d,} \mathrm{M.,} \mathrm{S} \mathrm{a} \mathrm{ling,} \mathrm{E.)}$ 1563

Diagnostische Maßnahmen bei der Prophylaxe der Rhesus-Sensibilisierung mit Immunglobulin-Anti-D (J u n g, K.) 1654 A. D.

Zur Prophylaxe der Rhesus-Sensibilisierung mit Immunglobulin-Anti-D ( $\mathrm{J} \mathrm{u} \mathrm{ng}, \quad \mathrm{K}$.) $1655 \mathrm{~A}$. Th.

Nebenwirkungen der Ovulationshemmer am Auge (Holl w i ch, F., Verbeck, B.) $1761 \mathrm{~K}$.

Röntgenreihenuntersuchung in der Schwangerschaft ( $\mathrm{Ne} \mathrm{u} \mathrm{m} \mathrm{a} \mathrm{n} \mathrm{n,} \mathrm{G.)} 2127$

Verhütung von Skelettfehlbildungen nach mütterlichem Blutverlust durch Elektrolytersatz ( $\mathrm{Gr}$ o t h e, W.) 2342

Vibrio fetus als Krankheitserreger des Menschen (U $11 \mathrm{~m}$ a n n, U.) 2399 UU.

Hormontherapie bei gynäkologischen Karzinomen (K e p p , R.) 2408

Endokrine Schutzmechanismen gegen Endometrium- und Mammakarzinome $(\mathrm{K} \mathrm{a} \mathrm{i}$ s e r, R.) 2467

Norethisteronönanthat. Untersuchungen während der Anwendung eines langwirkenden parenteralen Kontrazeptivum (Gilf $\mathrm{rich}$, H. J., N i e s chla g, E., D u d e ck, J., Overzi e r, C.) 2473

Einfluß oraler Ovulationshemmer auf klinisch-chemische Normalwerte (W il b e $\mathrm{rt}$, L.) $2522 \mathrm{~K}$.

Bestimmung der Bilirubinkonzentration im Fruchtwasser bei Rh-Inkompatibilitär (Reil, B., Schellong, G., Mast, H.) 2602

Vasopressin und Oxytocin ( $\mathrm{R}$ a u ch, R.) 2611 Ư.

\section{Geschwülste}

Hämangioperizytom ( $\mathrm{H} \mathrm{a} \mathrm{r} \mathrm{t} \mathrm{w} \mathrm{i} \mathrm{c} \mathrm{h} \mathrm{,} \mathrm{G.)} 1506$ Behandlungsergebnisse bei zufällig entdecktem und symptomlosem Bronchialkarzinom (B e r n d t , H.) 1559

Das Problem der Krebserkrankungen in der Vorstellung der Bevölkerung ( $\mathrm{N}$ e u $\mathrm{m}$ a $\mathrm{n} \mathrm{n}$, G.) $1581 \mathrm{~K}$.

Die Strahlenbehandlung des Bronchialkarzinoms (V i e t e n, H., G ü n th e r, D.) 1593

Uber primäre Sarkome des Hauptstammes der Arteria pulmonalis ( $\mathrm{C}$ a i n, H.) 1607

Die Bedeutung der Pleura- und Lungenbiopsie in der internen Diagnostik ( $M \ddot{u} r t z$, R., B e g e n a t, H.) 1724

Die Behandlung spontaner Hypoglykämien bei einem inoperablen Leberzellkarzinom durch Glucagon ( $M$ ü h e, E., S chrikk e r, K. T., R a i the l, D.) 1781

Verzögerte Reaktion von Morbus HodgkinLymphozyten auf Phytohämagglutinin (Havemann, K.) 1867
Die Effektivität einer präventiven Krebsbe kämpfung (O e s e r, H., R a c h, K.) 201 Thymektomie und Allergie ( $\mathrm{Geh} \mathrm{rm}$ an $\mathrm{n}$ G.) 2062

Paraneoplastische Polyneuropathie bei Thy muskarzinom (M i elke, F., W olter M.) 2102

Erhöht vorsorgliche Röntgenreihenunter suchung die Uberlebensrate beim Lungen krebs? (O e s e r, H., B e rnd t, H.,) 218( L. Z.

Die derzeitige Häufigkeit der einzelnet Lymphknotenerkrankungen in Schleswig Holstein (L e n n e r t , K.) 2194

Differentialdiagnostische Probleme bei Tuber kulomen des Gehirns (Möbi u s, W'.) 228

Indikationen, Möglichkeiten und Grenzen des selektiven Oberbaucharteriographie (P r e t e r, B., W ir th, W.) 2395 A. D.

Hormontherapie bei gynäkologischen Karzi. nomen ( $\mathrm{K} \mathrm{e} \mathrm{p} \mathrm{p,} \mathrm{R.)} 2408$

Allgemeine Grundlagen der Diagnostik vor Magen-Frühkarzinomen mit der Gastrokamera (O s h i m a, H.) 2440 A. D.

Behandlungsmöglichkeiten bei Hypophysentumoren (W e b e r, G.) 2442 A. Th.

Zur Effektivität von Krebsvorsorgeuntersuchungen ( $\mathrm{B}$ e r nd t, $\mathrm{H}$., O e s e r, H., $\mathrm{R}$ a c h, K.) 2452, 2453 L. Z.

Endokrine Schutzmechanismen gegen Endometrium- und Mammakarzinome ( $\mathrm{Ka} \mathrm{i}$ s e r, R.) 2467

Behandlung des multiplen Myeloms mit einem neuen Zytostatikum je-[1-Methyl-5-bis-( $\beta$ chloräthyl)-amino-benzimidazolyl-(2)]-buttersäure-hydrochlorid (A n g e r, G., Hes se, P., B a u f eld, H.) 2495

Kontrastmittelspeicherung in der Leber nach Fußlymphographie (Hepatographie) (T is m e r, R., Fri ed ma n n, G.) 2547

Zur Effektivität von Krebsvorsorgeuntersuchungen ( $\mathrm{Z} \mathrm{u} \mathrm{tz}$, H.-U.) 2569 L. Z

Erfahrungen mit »ultrahohen "Cyclophosphamid-Dosen an 42 Kranken mit soliden und lymphoretikulären Tumoren $(\mathrm{S} \mathrm{ch} \mathrm{u}$ bert, J. C. F., Fischer, M., Hilbert, L.) 2583

Primärer Hyperparathyreoidismus infolge multipler, eutopisch u. dystopisch gelegener Epithelkörperchenadenome (D i e th e l m, L., Gerok, W., K ü m m e rle, F. Pabst, K., Hennekeuser, H.-H.) 2593

Der Wilms-Tumor im ersten Lebensjahr. Insbesondere über 62 Nephroblastome des Neugeborenen (B a chmann, K. D., $\mathrm{K}$ röll, W.) 2598

Karzinogene Wirkung von Cyclamat? (Mend e n, E.) 2619

\section{Harnorgane}

Zur Behandlung der renalen Acidose mit Natriumbicarbonat und Ethacrynsäure (Q uellhorst, E., Scheler, F.) 1398

Indikationen zur Bestimmung der Reninaktivität im Blut ( $\mathrm{H}$ o d l e r, J.) 1417 A. D.

Hypercalciurie unter PAS-Calcium-Medikation als pathogenetischer Faktor der Urolithiasis 1. Mitteilung ( $\mathrm{Sch}$ ön e, D., $\mathrm{H}$ a u schild, G., Wässer, St.) 1491

Kryochirurgische Behandlungsverfahren in der Urologie (O pelt, B., Sökela nd, J., Ze ma n, N.) 1497 Ư.'

Bakteriurien in der Schwangerschaft. Epidemiologie, geburtshilfliche Komplikationen $\mathrm{u}$. Behandlungsergebnisse $(\mathrm{H}$ i $\mathrm{r} \mathrm{s} \mathrm{h}$, H. A., D e c k e r, R.) 1553 
Möglichkeiten und Grenzen hochdosierter Diuretikatherapie bei hydropischer Niereninsuffizienz ( $\mathrm{He}$ i d l a n d, A., K l ü $\mathrm{tsch}$, A., Moormann, A., Hennemann, H.) 1568

Die Beeinflussung der Hämodynamik durch arteriovenöse Fisteln bei Dialyse-Patienten (S i ll, V., Tilsne r, V., B a u d it z, W.) 1604

Hitzeberuf bei Nierensteinleiden (L u t z e y e r, W.) 1666

Beitrag der Nierenbiopsie zum nephrotischen Syndrom (B ohle, A., Kluthe, R., Sarre, H., Wehner, H.) 1673

Nierendurchblutung und Glomerulumfiltrat unter Dihydralazin-Wirkung (W ü r d i n g e r, H., A ust, W., M öll e r, U.) 1688 Suizid durch Genuß von Bananen (R i t z, E., A nd rass y, K., Z i e gle r, M., Kre m p i e n, B.) 1767 L. Z.

Das Goodpasture-Syndrom. Ein klinischer und pathologisch-anatomischer Beitrag (Kra us s, J., Red e n b a cher, M., Streicher, E.) 1820

Zum Problem der exakten Flüssigkeitsbilanz bei der Peritonealdialyse ( $\mathrm{K} \mathrm{r}$ a m e r, P., Quellhorst, E., Hen ing, H. V., Scheler, F.,) 1873

Möglichkeiten u. Probleme der Harndialyse (B und s c h u, H. D., D ü r r, F.) 1888

Osmotherapie bei Intoxikationen ( $\mathrm{Mertz}$, D. P.) 1896

Nitrofurantoin-Fieber (L ü b b e r s, P.) 1922

Hypercalciurie unter PAS-Calcium-Medikation als pathogenetischer Faktor der Urolithiasis 2. Mitteilung ( $\mathrm{Sch}$ ön e, D., $\mathrm{Ha} \mathrm{u}$ schild, G., Wässe r, St.) 1928

Behandlung der Pyelonephritis im Kindesalter (O e h m e, J.) $1939 \mathrm{~A}$. Th.

Der vesiko-ureterale Reflux ( $\mathrm{S}$ ö k e la nd, J., Moormann, J. G., O pelt, B.) 2053 Ü.

Zum Problem der exakten Flüssigkeitsbilanz bei der Peritonealdialyse (Engels ing, B.) 2059 L. Z.

Die Bedeutung der chirurgischen Vor- und Nachbehandlung bei Organtransplantationen (P i chlm a i e r, H., Edel, H. H., L a vender, R.) 2082

Diätetische Therapie bei Niereninsuffizienz (K l u th e, R., Q u irin, H.) $2112 \mathrm{~A}$. Th. Die künstliche Harnableitung ( $\mathrm{M}$ a y, P., S ök el and, J.) 2115 U.

Autonomer Hyperparathyreoidismus infolge Nebenschilddrüsenadenom nach Nierentransplantation (Kistler, H. J., Scheit lin, W., Mayor, G., Uehlinger, E.) 2140

Toxische Hypercalcämie und reversible Weichteilverkalkungen in der Polyuriephase nach akutem Nierenversagen ( $\mathrm{L}$ a n ge, $\mathrm{H}$., Gossmann, H. H., S e ybold, D., Vog t, O., P f a b, R., D o m browski, H.) 2155

Struktur und Funktion des juxtaglomerulären Apparates der Niere unter geordneten Bedingungen ( $\mathrm{C}$ a i n, $\mathrm{H}$., $\mathrm{Kr}$ a u s, B.) 2173 U.

Zum Problem der sogenannten Transplantationslunge $(\mathrm{B} \mathrm{e} \mathrm{ck} \mathrm{e} \mathrm{r}, \mathrm{H}$. D., $\mathrm{H}$ ü b n e $\mathrm{r}$, G., Eigler, F. W., S i e be rt, H. G:) 2202

Erfahrungen mit einer einfachen Methode zur quantitativen Keimbestimmung aus dem Urin (W ill e, L., W in t e r, J.) 2223

Plasma-Renin bei akuter und chronischer Niereninsuffizienz und Hämodialyse ( $\mathrm{S} \mathrm{ch} \mathrm{r} \ddot{\mathrm{o}}$ der, E., Herms, W., We tzels, E., D u me, Th., Grabense e, B.) 2262

Behandlung der Pyelonephritis im Kindesalter (W i o n tze k, H.) 2356 L. Z.
Behandlung des »akuten « Harnsteines (V a h le n s i e c k, W.) 2381

Nitrofurantoin-Fieber ( $\mathrm{L}$ a u b e $\mathrm{n} \mathrm{th}$ a l, F.) 2523 L. Z.

Behandlung der interstitiellen Zystitis ( $\mathrm{S}$ ö k e land, J.) 2620

Uber eine Familie mit Goodpasture-Syndrom, Alport-Syndrom und Asthma bronchiale (M üntefering, H., Hilscher, W., $\mathrm{M}$ i es e, A.) 2637

Die Wirkung von Cumarin-, Indandion- und Benzofuran-Derivaten auf die renale Harnsäureausscheidung ( $Z$ öllñer, N., Gröb n e r, W.) $2652 \mathrm{~K}$.

Zur Bestimmung von Glómerulumfiltrat und effektivem renalem Plasmafluß. Prüfung eines vereinfachten Einmalinjektionsverfahrens (W e id m a n n, P., Fis che r, W. M ülle r-Duysing, W., Horst, W., $\mathrm{S}$ chö n beck, M., We rn ing, C., $\mathrm{H}$ üls, W., Si e genthaler, W.) 2685

\section{Haut- und Geschlechtsorgane}

Die rosacea-artige Dermatitis: ein neuartiges Krankheitsbild. Periorale Dermatitis (S t e i gl e d e r, G. K.) 1393

Noch einmal: Lyell-Syndrom ( $\mathrm{Z}$ i m m e $\mathrm{r}$ m a n n, U.) $1422 \mathrm{~L}$. Z.

Psoriasis am behaarten Kopf (B ra u n F a lc o, O.) 1466

Sexualstörungen durch Valium (C o p e $r, H$.) 1467

Zur Fieber- und Penicillinbehandlung der progressiven Paralyse in neuropathologischer Sicht (Q u a n d t, J., S o m m e r, H.) 1486

Die Diagnose der Porphyria cutanea tarda (Filippini, L., Strohmeyer, G.) 1577 A. D.

Kontaktallergien durch Nifurprazin (Carofur) (B r a u n, W.) 1685

Dermatitis seborrhoica infantum (B a n dm a n n, H.-J.) 1841

Hämangiome (S chn yde r, U. W.) 1990 A. D.

Perkutane Alkoholresorption ( $\mathrm{S} \mathrm{ch} \mathrm{uh,} \mathrm{F.)}$ 2008

Erythematodes-Provokation durch Goldtherapie wegen primär-chronischer Polyarthritis (G o e r z, G.) 2040

Kälteurtikaria und Kininsystem (D u c k , H.J.) 2059 L. Z.

Extragenitale Trichomonadeninfektion (E y e r, H.) 2525

\section{Herz und Kreislauf}

Antikoagulantientherapie bei Mitralstenose ( $\mathrm{C} \mathrm{ch} \mathrm{mutzle} \mathrm{r,} \mathrm{R.)} 1424$

Die hypokaliämische metabolische Alkalose (E u ch e nh of er, M., S tre icher, E., Würz, H., M eurer, K. A., S te in e r, B., D ü r r, F., K a u f m a n n, W.) 1441

Ergebnisse der direkten und indirekten Isthmusplastik bei Kindern und Jugendlichen unter besonderer Berücksichtigung des Anastomosenwachstums ( $\mathrm{He} \mathrm{hrle} \mathrm{in,} \mathrm{F}$. W.) 1522

Die Diagnostik der muskulären subvalvulären Aortenstenose (W e n ge r, R.) 1537 A. D.

Die Therapie der muskulären subvalvulären Aortenstenose (W e n g e r, R.) 1539 A. Th.

Die Beeinflussung der Hämodynamik durch arteriovenöse Fisteln bei Dialyse-Patienten (S i ll, V., T ils s e r, V., B a u d it z, W.) 1604

Úber primäre Sarkome des Hauptstammes der Arteria pulmonalis ( $\mathrm{C}$ a i n , H.) 1607
Die elektromagnetische Registrierung von Stromzeitvolumina ( $\mathrm{M}$ e is ner, $\mathrm{H}$. M e s s m e r, K.) 1621

Intramuraler Infarkt (H e in e c k e r, R.) 1625

Kardiogener Schock nach Myokardinfarkt. Therapieergebnisse mit Isoprenalin und niedermolekularem Dextran ( $\mathrm{N}$ a g e r, F., Lichtlen, P., Rösli, R., S teinbrunn, W., Gutschmidt, A., Reutter, F., B lankart, R., Reichmuth, J., Burger, H., Af folter. H., D ebrunner, F., Ritz, R., Ueh linge r, A., M e y e r, H. A.) 1640

Framingham-Studie (S c hl i e r f, G.) 1667

Die Wirkung von Octapressin unter Narkosebedingungen auf Pfortaderdruck, arteriellen Blutdruck und zentralen Venendruck (H e n n eb e r g, U., E ck a r t, J. P., H ̈̈ $\mathrm{r}$ in $\mathrm{g}, \mathrm{R}$.) 1679

Behandlung des Hitzschlags ( $M$ ü 11 e r, W: A., Kerkhoven, P., Truninger, B.) 1695

Beeinflussung der Angina pectoris durch Carotissinusnerven-Reizung. Erfahrungen mit einem elektronisch gesteuerten Carotissinus-Stimulator ( $\mathrm{S} \mathrm{ch}$ a e d e, A., W a g n e $r$, J.) 1717

Pharmakodynamische Phonokardiographie (R a u tenburg, H. W.) 1750 A.D.

Behandlung des kardiogenen Schocks (P a b s t, K., Win ck el m a n n, G.) 1752 A. Th.

Die Myokardiopathien (M e y e r , J.) 1755 Ư.

40 Jahre Herzkatheterismus ( $\mathrm{S} \mathrm{chad} \mathrm{e}$ w ald t , H.) 1768

Hypokaliämiezeichen im pathologischen Elektrokardiogramm ( $\mathrm{H}$ e in e c k e r, R.) 1841

Die kleinherdige hypoxidotische Herzmuskelnekrose (P o c h e , R.) 1851

Herzinfarkt nach Tetanusschutzimpfung ( $\mathrm{Sch}$ a c d e, A.) 1895

Die Revaskularisation des Myokards bei koronarer Herzkrankheit ( $\mathrm{H}$ e g e $\mathrm{m}$ a $\mathrm{n} \mathrm{n}$, G., B a ch m a n n, K., D it t r i c h, H.) 1903

Transpulmonale arterio-venöse Fistel zwischen Körper- und Lungenkreislauf (v. Eg id y, H., Habighorst, L. V., T a lk e, H.) 1917

Die Diagnose des akuten Mesenterialgefäßverschlusses ( $\mathrm{H} \mathrm{u} \mathrm{b} \mathrm{e} \mathrm{r,} \mathrm{F.} \mathrm{B.)} 1937$ A. D.

Ist die Verwendung von Oleum camphoratum noch zeitgemäß! (B r ü g m a n n, E.) 1945 L. Z.

Blutdruckmessung und Armumfang (A n $\mathrm{s} \mathrm{ch}$ ü t z, F.) 1950

Rezidivierende Polychondritis mit Aortenaneurysmen. Jaksch-Wartenhorst-v. Meyenburg-Altherr-Uehlinger-Syndrom ( $\mathrm{L}$ a n $\mathrm{g}$, H. D., M üll e r, D., F in k e, J.) 2033

Kombinationstherapie: Digitalis und Calcium (G r e eff, K.) 2062

Intermittierender Verschluß der Arteria poplitea durch zystische Adventitiadegeneration (E hringer, H., D e n ck, H., W u k e t i ch, St., B run ne r, E.) 2107

Circuit-Training im Schulsport (Hollm a n n, W.) 2127

Der chronische idiopathische Perikarderguß (W i n k, K., H a g e r, W.) 2160

Myelofibrose und Osophagusvarizen (W o h le n b e r g, H.) 2163

Die Diagnose des senilen Herzamyloids (S t e in m a n n, B.) 2170 A. D.

Die Behandlung der hypotonen Kreislaufregulationsstörung (D eli u s, L.) 2172 A. Th.

Pathologisches Belastungs-Elektrokardiogramm bei Orthostasereaktion ( $\mathrm{Hol} z$ m a n n, M.) 2182 
Hirndurchblutung bei Digitalistherapie (Hot t s te in, U.) 2182

Asystolischer Herzstillstand bei Achalasie (Rösch, W., B a chmann, K., Otten j a n n, R.) 2191

Schwere traumatische Hämolyse und Endokarditis bei Faszienklappenplastik der Aorta. Korrektur durch Kugelklappe (S t r a u b, P. W., R ot h li n, M.) 2212

Polymyalgia rheumatica (K a i s e r, H.) 2232

Thrombolysetherapie (S c h i m p f, Kl.) 2292 A. Th.

Gefahren bei der Streptokinase-Behandlung von Herzinfarkten (B a u m a n n , G.) 2296 L. Z.

Orthostatische Blutdrucklabilität beim Phäochromozytom (W e r n in g, C.) 2299

Die chirurgische Behandlung des akuten Arterienverschlusses (Voll m e r, J., L a u b a c h, K., Grus s, J. D.) 2315

Erfahrungen mit dem Einschwemmkatheter "Pulmocath" (K le m p t, H.-W., Noder, W.) 2345 A. D.

Medikamentöse Therapie peripherer arterieller Durchblutungsstörungen ( $\mathrm{B}$ olling e $\mathrm{r}$, A.) $2347 \mathrm{~A}$. Th.

Blutdruck bei ambulanter Untersuchung (M e e s m a n n, W.) 2359

Digitalisresorption bei Rechtsherzinsuffizienz (Gill m a n n, H.) 2359

Zur Therapie der zerebralen Insuffizienz (Grill, P., B roich e r, H.) 2429

Wadenschmerzen (M üll e r, N.) 2526

Behandlung mit $\beta$-Rezeptorenblockern ( $\mathrm{L}$ y d ti n, H.) 2560 A. Th.

Zur Koronarreserve des Menschen (Doll, E., K e u l, J.) 2563 Ü

Bestimmung der Herzgröße ( $\mathrm{S} \mathrm{ch}$ a e d e , A.) 2571

Therapie zerebralsklerotischer Verwirrtheitszustände (R ü m m e le, W.) 2571

Chirurgische Resultate bei muskulärer Subaortenstenose (R o th lin, M., W ir z, P., Preter, B., Gat tike r, H., Senning, A.) 2579

Diagnostische Maßnahmen bei Verdacht auf Lungeninfarkt (M o r a w e t z, F.) 2605 A. D.

Die Beteiligung innerer Organe bei der Polyarthritis chronica progressiva ( $\mathrm{B}$ a i e $r, M$.) 2644 A. D.

Die medizinische Behandlung der koronaren Herzkrankheit (S chweiz e r, W.) 2646 A. Th.

Uber die Beeinflussung von Herzrhythmusstörungen durch $\alpha$-Propranolol (A m o r, H., Di enstl, F., Judmai er, G., Schwing shack1, H.) 2669

Diagnose der Fettembolie (P e l z l, H.) 2690 A. D.

\section{Infektionskrankheiten, Immunität}

Kombinierte spezifische Immunsuppression (G ü t h e r, O.) $1421 \mathrm{~K}$

Cholera- u. Typhus-Schutzimpfung (Stickl, H.) 1425

Lysismuster und Antibiogramm von Staphylokokken bei septischen Krankheitsbildern (M a t z, K., S c h a s s a n, H.-H.) 1448

Die Therapie der Filariose ( $\mathrm{R}$ h o m b e r g, F.) $1457 \mathrm{~A}$. Th.

Malaria als Importinfektion (S tille, W., $\mathrm{Kalt}$ w a s s r, P.) 1518

Perivenöse Markdestruktionen bei eitriger Meningitis ( $\mathrm{t}$ a $\mathrm{m} \mathrm{m} \mathrm{l} \mathrm{e} \mathrm{r,} \mathrm{A.)} 1529$

Möglichkeiten zur Hemmung der Virusvermehrung (E g g e r s, H. J.) 1540 U.

Desinfektion nach Mumps-Meningitis (S p i e s s, H.) 1585
Die Bedeutung der Mumpsmeningitis ( $\mathrm{R}$ a dl, H.) 1599

Klimakammerbehandlung bei Pertussis (K ̈̈ h n k e, F.) 1624 L. Z.

Rötelnschutzimpfung (V i v e 11, O.) 1626

Fremdviren in Impfstoffen ( $\mathrm{M}$ a j e r, M.) 1617 Ư.

Seuchenhygienische Maßnahmen beim Bolulismus ( $\mathrm{R} \mathrm{e} \mathrm{plo} \mathrm{h,} \mathrm{H.)} 1666$

Das Tollwutvirus (K u w e r t, E.) 1697

Tetanusauffrischimpfung (S p i e s s, H.) 1707

Erkältung als Infektbahnung (V i ve l I, O.) 1771

Ponndorf-Impfung ( $\mathrm{N} \mathrm{e} \mathrm{u} \mathrm{m} \mathrm{a} \mathrm{n} \mathrm{n,} \mathrm{G.)} 1772$ Infektionen mit Pasteurella multocida nach Tierbissen (Stille, W., Stoll, L., Helm, E.) 1816

Zur Frage der posthepatischen Leberzirrhose (W ild hi rt, E., Franke n, F. H.) 1838 L. Z.

Segmentale motorische Paresen beim Zoster ( $\mathrm{S} \mathrm{ch} \mathrm{li} \mathrm{a} \mathrm{c} \mathrm{k,} \mathrm{H.,} \mathrm{S} \mathrm{c} \mathrm{h} \mathrm{n} \mathrm{e} \mathrm{i} \mathrm{d} \mathrm{e} \mathrm{r,} \mathrm{H.)} 1861$ Herzinfarkt nach Tetanusschutzimpfung ( $\mathrm{S}$ c h a e d e, A.) 1895

Die je-Glutamyl-Transpeptidase-Aktivität im Serum bei hepatobiliären Erkrankungen (Szusz, G., Rosenthal, P., Fritzs che, W.) 1911

Die "Herpangina «-Epidemie 1967/68 (ECHO $30 / 6 / 3$ ). Bericht über 590 klinisch beobachtete Fälle im Säuglings- und Kindesalter (Ke u th, U., Esser, I., Wilhel $\mathrm{mi}$, J., Wil he l m i, I.) 1959

Komplementbindende Antikörper gegen das Zytomegalievirus bei Kindern und Erwachsenen (P e ll e r, P., G o e t z, O.) 1966

Quantitative Komplementanalysen (C'3) mit der radialen Immundiffusion bei Erkrankungen mit gestörter Immunitätslage (B l äker, F., Fischer, K., Witte, P.) 1978

Therapie der Lungenmykosen (We g m a n $\mathrm{n}$, T.) 1992 A. Th.

Verhütet Bettruhe die Mumps-Meningitis? (I1l i g, H.) 2004 L. Z.

Thymektomie und Allergie ( $\mathrm{Gehr}$ m a n $\mathrm{n}$, G.) 2062

Neurologische Erkrankungen durch Enteroviren bei Kindern (W ind orfer, A., S i t z m a n n, F. C.) 2071

Echo-9-Infektion in einer Landgemeinde. Eine epidemiologische und virologische Studie (G e r th, H.-J., S ch oo p, H. D., O ch s, U., F i s cher, K.) 2076

Pneumocystis-carinii-Pneumonie beim $\mathrm{Er}$ wachsenen (F i s cher, R., B e cker, H. D., J o is t, J. H., T is m e r, R.) 2135

Die Neuraminidase als pathogenetischer Faktor bei Pneumokokken-Infektionen (M ü 1 le r, H. E.) 2149

Zur Prognose der chronischen Hepatitis. I. Formen und Entwicklungsstadien (V i d o, I., Selmair, H., Wildhirt, E., Ort mans, H.) 2215

Zur Prognose der chronischen Hepatitis. II. Verlaufsdauer (S elm a ir, H., Vid o, I., Wildhirt, E.) 2220

Röntgenologische Lungenbefunde bei septischen Erkrankungen (D u n ker, S., Still e, W.) 2226

Möglichkeiten und Grenzen der modernen Leberkoma-Therapie. Austauschtransfusion, Leberperfusion und Leberersatz (Ko m m e re ll, B.) 2235 Ư.

Australia-Antigen und Virushepatitis (S c r i b a, M.) 2294 U.

Resochin bei chronisch-aggressiver Hepatitis (F r a n k e n, H. H.) 2299

Das reversible procainamidinduzierte Lupuserythematodes-Syndrom ( $G$ ü $n t h$ e $r$, $R$., A s a m e r, H., D it t r i ch, P.) 2338
Zur biologischen Aktivität pathogener Staphylokokken (J e l j a s z e w i c z, J., P u lvere r, G.) 2349 Ư.

Unspezifische erworbene Makrophagenresistenz (G ü n th e r, O.) $2355 \mathrm{~K}$.

Therapie der chronischen Hepatitis ( $\mathrm{F} \mathrm{r}$ a $\mathrm{n}$ ke n, F. H.) 2397 A. Th.

Vibrio fetus als Krankheitserreger des Menschen (U11 m a n n, U.) 2399 U.

Pocken: Frühimpfung oder Impfung im zweiten Lebensjahr? (E h r e n g u t, W.) $2403 \mathrm{~K}$

Zur Ampicillin-Therapie des Keuchhustens (S i m o n, C., B on t e m ps, M., Wi e se K., S c h e w i o r - R o land, R.) 2435

Langzeitbehandlung von Immunopathien unter besonderer Berücksichtigung immunsuppressiver Medikamente ( $\mathrm{Sch}$ e if $\mathrm{far} \mathrm{h}$, F., W a rn a tz, H.) 2444 U.

Sepsis und Meningitis durch Vibrio fetus (S till e, W., H el m, E. B.) 2484

Bakteriologische Untersuchungen mit Carbenicillin (B lasius, Ch., M enz, H.-P., $\mathrm{S}$ a n d e r, J.) 2501

Zur Antibiotikatherapie der Nocardiose (S c ha a l, K. P., Le is ch i k, W.) 2505 Mykogene Allergie. Schimmelpilz- und Pilzsporen als Ursache allergischer Atemwegsleiden (V i r c h o w, Chr.) 2508

Möglichkeiten der spezifischen Grippeprophylaxe (G e r th, H.-J.) 2510 A. Th.

Extragenitale Trichomonadeninfektion (E y e r, H.) 2525

Virusinfektionen und Parkinson-Syndrom (U m b a c h, W.) 2525

Tuberkulinprobe in der Differentialdiagnostik ( $\mathrm{He} \mathrm{s} \mathrm{s,W.)} 2568$

Tollwutschutzimpfung von Hunden (S chlies ser, Th.) 2620

Hühnerzüchterlunge ( $\mathrm{B}$ ü t i k of e r, E., d e W e c k, A. L.) 2627

\section{Innere Sekretion, Vitamine}

Die hypokaliämische metabolische Alkalose (E u che nh of er, M., S tre ich e r E., W ürz, H., M e ure r, K. A., S t e i n e r, B., D ü r $r$, F., K a u f m a n n, W.) 1441

Die diagnostische Wertigkeit des Trijodthyronin-Tests (S c r i b a , P. C.) 1455 A. D.

Funktionspsychosen als Manifestationen der $\mathrm{B}_{1 \text { - }}$-Avitaminosen (W i e c k, H. H., P r i. billa, W., Heerklotz, B.) 1473

Hypoglycaemia factitia (T o r n o w , P.) 1502 L. Z.

Schwerwiegende Fehlbeurteilungen bei der Urinuntersuchung auf Glucose mit »Schnelltesten " (O l b in g, H., W e $11 \mathrm{~m}$ a n n, P.) 1566

Diabetes mellitus durch Gonadotropintherapie? (H o h e nf ell n e r, R.) 1626

Intravenöser Glucosebelastungstest $(\mathrm{M} \mathrm{e} \mathrm{h}$ n e r t , H.) 1706

Hafertage beim Diabetes (S a u e r, H.) 1771

ACTH- oder Glucocorticoid-Therapie? (K a i s e r, H.) 1771

Vitamin $B_{12}$ bei Leberleiden (D e $\mathrm{m} \mathrm{l}$ in $\mathrm{g}, \mathrm{L}$.) 1771

Funktionspsychosen als Manifestationen der $\mathrm{B}_{12}$-Avitaminosen ( $\mathrm{R}$ i n g w a l d, E.) 1945

Diabetische Enteropathie ( $\mathrm{M}$ e h n e r t, H.) 1950

Vitamin D. Neue Ergebnisse zum Wirkungsmechanismus von Cholecalciferol (W o lf H., del S o l a r, E.) 1996

Endogener idiohypophysärer Diabetes mellitus (W a ldhäu s l, W., B u ri a n, K., B e ringer, A.) 2028 
Jiagnostik des sekundären Hyperaldosteronismus (W e rning, C., Siege $\mathrm{n} t \mathrm{~h}$ ale r, W.) 2049 A. D.

Therapie des sekundären Hyperaldosteronismus (W e rning, C., S i e g e $n$th a le $r$, W.) $2051 \mathrm{~A}$. Th.

Autonomer Hyperparathyreoidismus infolge Nebenschilddrüsenadenom nach Nierentransplantation (K is tle r, H. J., Scheit lin, W., Mayor, G., Uehlinger, E.) 2140

Angeborenes adrenogenitales Salzverlustsyndrom mit Entfaltung einer Prostata beim weiblichen Säugling ( $\mathrm{S} \mathrm{ch} \mathrm{u} \mathrm{h} \mathrm{m} \mathrm{a} \mathrm{n} \mathrm{n,} \mathrm{R.)}$ 2229

Normokaliämischer primärer Aldosteronismus ( $\mathrm{H}$ ä n z e , S.) 2359

Freies Thyroxin und proteingebundenes Jod im Serum bei Eu- und Hyperthyreose (Krüs ke m per, H. L., Herrma n n, J.) 2386

Schilddrüsendiagnostik und jodhaltige Zahnpasta (H o r s t e r , F. A.) 2408

Behandlungsmöglichkeiten bei Hypophysentumoren (W e b e r, G.) 2442

Endokrine Schutzmechanismen gegen Endometrium- und Mammakarzinome $(\mathrm{K} \mathrm{a}$ i s e r, R.) 2467

Norethisteronönanthat. Untersuchungen während der Anwendung eines langwirkenden parenteralen Kontrazeptivum ( $\mathrm{G}$ il $\mathrm{f} \mathrm{r} \mathrm{i} \mathrm{ch,}$ H. J., $\mathrm{N}$ i e s chlag, E., D u d e ck, J., Overzi e r, C.) 2473

Klinisch-experimentelle Untersuchung zur Hemmwirkung verschiedener Corticoide (Hoch heuser, W., Gerb, A., M ül ler-Bardorff, M., Thiele, H.) 2488

Klinischer Beitrag zur Frage der optimalen Darreichung von Schilddrüsenhormonen (E i chenbusch, W., Ha u t, E. L a h r t z, Hg., L e y b old, K.) 2478

Nahrungsbedarf diabetischer Kinder

(S a ch s se, R., S a ch s s e, B., J a h n ke, K., D a w e k e, H.) 2535

Primärer Hyperparathyreoidismus infolge multipler, eutopischer und dystopisch gelegener Epithelkörperchenadenome ( $D$ i e $t$. h e l m, L., Gerok, W., Kü m m e r le, F., P a b s t, K., H e n ne ke u s e r, H.-H.) 2593

Vasopressin und Oxytocin ( $\mathrm{R} \mathrm{a} \mathrm{u} \mathrm{ch,} \mathrm{R.)}$ 2611 Ú.

Peritonealdialyse bei thyreotoxischer Krise ( $\mathrm{H}$ e rrma n n, J., B e is e $\mathrm{nh}$ erz, W., Gillich, K. K., Jester, H. G., Kluge, R., Nis sen, P., Kr ü skem p e r, H. L.) 2615 L. Z.

Uber das Hyperthyreose-Risiko bei iatrogener Jodzufuhr ( $\mathrm{S}$ ch n e id e r, C., S t e p ha n, G., $S$ u w e l a c k, M.) 2631

Beitrag zum renalen Kaliumverlustsyndrom. Bericht über einen Fall von geleugnetem Diuretika-Abusus (Adolph , H.-D., I r m s cher, K., Solbach, H. G.) 2634

Zuckeraustauschstoffe bei labilem ketoacidotischem Diabetes ( $\mathrm{M} \mathrm{e} \mathrm{h} \mathrm{n} \mathrm{e} \mathrm{r} \mathrm{t,} \mathrm{H.)} 2659$

Proteinurie bei Glibenclamid-Behandlung? (K ü h n a u, J.) 2703

\section{Kinderheilkunde}

Mikrochemische Urineisenbestimmung be Säuglingen und Kindern. Untersuchungen zur Urineisenbestimmung vor und nach Deferoxamin-Gabe bei Gesunden, Patienten mit Eisenmangel und Eisenüberladung (M a a s, B., N e th, R., G o s chen h of e r, D., S c häf e r, K. H.) 1410
Glykogenose Typ II (Pompesche Krankheit) mit $\alpha$-Amylase- und Hyaluronidase-Mangel (P l a t t, D., P l a t t, M.) 1414

Biochemie und Klinik der Glykogenosen (Plat t , D.) $1420 U$

Zur Frage der Anti-Rh $(\mathrm{D})$-Prophylaxe in der Schwangerschaft ( $\mathrm{H}$ a e r in g, M.) 1433

Zur Wirkung von Immunglobulinanti-D im Rh-positiven Organismus. Úbersicht über bisher beobachtete Fehlanwendungen des Immunglobulins-anti-D ( $\mathrm{N}$ i e d e $\mathrm{r} h$ of $f$, N., Schneider, J., Stachow, P., $\mathrm{K} \ddot{\mathrm{u}} \mathrm{nz}$ e r, W.) 1437

Fernsehschäden bei Kindern (U Id a 11, B.) 1453

Die Bedeutung enteralen Lysozyms für den Säugling (B r a u n, O. H.) 1458

Legasthenie und Ganzwortmethode (I s l a r, K.-G.) 1462 L. Z.

Hypercalciurie unter PAS-Calcium-Medikation als pathogenetischer Faktor der Urolithiasis. 1. Mitteilung ( $\mathrm{S} \mathrm{ch}$ ö n e, D., $\mathrm{H}$ a u s child, G., W ä s s e r, S t.) 1491

Ergebnisse der direkten und indirekten Isthmusplastik bei Kindern und Jugendlichen unter besonderer Berücksichtigung des Anastomosenwachstums ( $\mathrm{H}$ e h $\mathrm{rl}$ e in, $\mathrm{F}$. W.) 1522

Die Diagnostik der muskulären subvalvulären Aortenstenose (W e n g e r, R.) 1537 A. D.

Die Therapie der muskulären subvalvulären

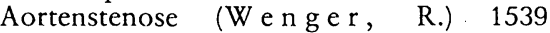
A. Th.

Perinatale Mortalität von Kindern mit Distress-Hinweisen vor dem Einsatz neuzeitlicher geburtshilflicher Úberwachungsverfahren (P a k z a d, M., S a l i ng, E.) 1563

Schwerwiegende Fehlbeurteilungen bei der Urinuntersuchung auf Glucose mit »Schnelltesten « (O l b ing, H., W ell $\mathrm{m}$ a n $n$, P.) 1566

Desinfektion nach Mumps-Meningitis (S p i e s s, H.) 1585

Die Bedeutung der Mumpsmeningitis ( $\mathrm{R}$ a d l, H.) 1599

Klimakammerbehandlung bei Pertussis (K ü h n k e, F.) 1624 L. Z.

Rötelnschutzimpfung (V i v e ll, O.) 1626

Diagnostische Maßnahmen bei der Prophylaxe der Rhesus-Sensibilisierung mit Immunglobulin-Anti-D (J u n g, K.) 1654 A. D.

Zur Prophylaxe der Rhesus-Sensibilisierung mit Immunglobulin-Anti-D ( $\mathrm{J} \mathrm{u} \mathrm{g}$, K.) $1655 \mathrm{~A}$. Th.

Fremdviren in Impfstoffen ( $\mathrm{M}$ a y e $\mathrm{r}, \mathrm{M}$.) 1617 Ư.

Beitrag der Nierenbiopsie zum nephrotischen Syndrom (B ohle, A., Kl u the, R., S a r re, H., We hn er, H.) 1673

Zur Diagnose der Mucoviscidose (zystische Fibrose) (S t e p h a n, U., B r e h m, G.) 1677

Tetanusauffrischimpfung (S p i e s s, 1707

Pharmakodynamische Phonokardiographie (R a u t e n bu r g, H. W.) 1750 A. D.

Colonresektion bei schwerer Hämophilie A unter dem Schutz von antihämophilen Kryopräzipitaten (B r ü s t e r, H., R i n g. ler, W., Glassner, K., Ri ech, P.-Ch r., Hi m m elb a ch, E.) 1799

Klinische Differenzierung des asthmatischen Syndroms (W e r n e r, M.) 1802

Hepatozellulärer Ikterus nach wiederholter intravenöser Infusion von Fettemulsionen bei Kindern mit ausgedehnter Darmresektion $(\mathrm{O} l \mathrm{~b}$ ing, H., Engels, A.-M. D ö n g e s, K. G.) 1825
Dermatitis seborrhoica infantum ( $B$ a n $d$ m a n n, H.-J.) 1841

Borsäure bei Säuglingen (I p p e n, H.) 1841

Katamnestische Erhebungen bei CoeliakiePatienten (W a g n e r, A., K o l b, P. J.) 1856

Beitrag zur Frage der Vitamin-D-Stoßbehandlung. Untersuchungen über den Einfluß des Vitamins D auf den Bindegewebsstoffwechsel und den Mechanismus der ossären Gewebsverkalkung ( $\mathrm{J} \mathrm{ung} \mathrm{e-H} \mathrm{üls} \mathrm{ing,}$ G., R a ve, O., W a g n e r, H., H ü th e r, W., Hilgen berg, F.) 1877

Therapie der Hämophilie ( $\mathrm{tr}$ a u b, P. W.) 1885 A. Th.

Legasthenie und Ganzwortmethode ( $\mathrm{B}$ a u e $\mathrm{r}$, J.) 1893 L. Z.

Hypercalciurie unter PAS-Calcium-Medikation als pathogenetischer Faktor der Urolithiasis. 2. Mitteilung ( $\mathrm{S} \mathrm{ch}$ ö n e, D., $\mathrm{H}$ a u s child, G., W ässer, St.) 1928 Behandlung der Pyelonephritis im Kindesalter (O e h m e, J.) 1939 A. Th.

Enterale Hämoglobinresorption ( $\mathrm{K}$ ü n z e r W.) 1950

Vererbbarkeitsrisiko des angeborenen Klump. fußes ( $\mathrm{M}$ a u , H.) 1950

Die »Herpangina «-Epidemie 1967/68 (ECHO $30 / 6 / 3$ ). Bericht über 590 klinisch beobach. tete Fälle im Säuglings- und Kindesalter (Ke u th, U., Esser, I., Wilhel mi, J., W il he l m i, I.) 1959

Komplementbindende Antikörper gegen das Zytomegalievirus bei Kindern und Erwachsenen (P e l l e r, P., G o e t z, O.) 1966

Quantitative Komplementanalysen (C'3) mit der radialen Immundiffusion bei Erkrankungen mit gestörter Immunitätslage (B l äk e r, F., Fis che r, K., W it t e, P.) 1878

Quantitative Mineralsalzbestimmung an kindlichen Skelett (Schuster, W. Reiss, K. H., K r a m e r, K.) 1983

Angeborene pulmonale Lymphangiektasien Bericht über 4 Fälle (Gle is sner, $P$., A s a n te, F., S chubert, G. E.) 1987

Hämangiome (Schnyder, U. W.) 1990 A. D.

Zur Induktions- und Intervall-Behandlung der Leukämien von Kindern und Jugendlichen (O ehme, J., Meyer-Beek, D.) 1993 Ư.

Vitamin D. Neue Ergebnisse zum Wirkungsmechanismus von Cholecalciferol (Wo lf H., d el S o l a r, E.) 1996

Thiamin-bedürftige megaloblastische Anämie (Ni ed e rhoff, H., K ünzer, W.) $2000 \mathrm{~K}$.

Verhütet Bettruhe die Mumps-Meningitis? I $11 \mathrm{ig}$, H.) 2004 L. Z.

Therapie bei rheumatischem Fieber ( $\mathrm{S}$ ü d. h of , H.) 2007

Der vesiko-ureterale Reflux ( $\mathrm{S}$ ök e l a nd, J., M o ormann, J. G., O pelt, B.) 2053

Neurologische Erkrankungen durch Enteroviren bei Kindern (W in dorfer, A. S i t z m a n n, F. C.) 2071

Echo-9-Infektion in einer Landgemeinde. Eine epidemiologische und virologische Studie (G e r th, H.-J., S choop, H. D., Och s, U., F i s c he r, K.) 2076

Uber katamnestische Untersuchungen bei jugendlichen Coeliakie-Patienten. Ein Beitrag zur Pathologie des Jugendalters (W e ber, A., Riecken, E. O., $M$ artin i, G. A.) 2206

Erfahrungen mit einer einfachen Methode zur quantitativen Keimbestimmung aus dem Urin (W ill e, L., W in t e r, J.) 2223 
Angeborenes adrenogenitales Salzverlustsyndrom mit Entfaltung einer Prostata bein weiblichen Säugling ( $\mathrm{S} \mathrm{ch}$ u h m a n n, R.) 2229

Knochenmarksschädigung bei immunsuppressiver Therapie der juvenilen rheumatoiden Arthritis und des Still-Syndroms mit Azathioprin (K ö $11 \mathrm{e}, \mathrm{G}$.) 2268

Elektrophoretische und immunologische $\mathrm{Be}$ funde beim Marfan-Syndrom ( $\mathrm{S}$ u s $\mathrm{ch} \mathrm{k}$ e, J., Heilm ann, K., Murken, J.-D., St e h r, K.) 2289

Zytochemische Differenzierung und Krankheitsverlauf akuter Leukämien des Kindesalters (B e c kma nn, H., N e th, R. L a n d beck, G., M a a s, B., Sch midt $\mathrm{k} \mathrm{e}, \mathrm{H}$.) 2320

Zur Pathologie der Osteopathie nach Heparinbehandlung (S chuster, J., $M$ ei e rRug e, W., E g l i , E.) 2334

Behandlung der Pyelonephritis im Kindesalter (W i o n t z e k, H.) 2356 L. Z.

Pocken: Frühimpfung oder Impfung im zweiten Lebensjahr? (E h r e ng u t, W.) 2403 $\mathrm{K}$.

Das Di-Gugliemo-Syndrom im Kindesalter (D rescher, J., Hansen, H. G., A l t h of $f, H ., G r a u c o b$, E.) 2415

Zur Ampicillin-Therapie des Keuchhustens (S i m o n, C., B o n t e m p s, M., W i e se, K., S c h e w i o r - R ol a n d, R.) 2435

Die Vor- und Nachteile der Bauchlage bei Neugeborenen und jungen Säuglingen (G l e is s, J.) $2449 \mathrm{~K}$.

Nahrungsbedarf diabetischer Kinder (S a chsse, R., S a chs se, B., J a h n ke, K., D a w e ke, H.) 2535

Neue Behandlungswege bei großen chirurgischen Eingriffen an Patienten mit Hämophilie A (W e d e ll, J., $R$ in $g$ le $r$, W., B rüste r, H., R i e ch, P. Chr., Göbel, U., P fizner, W.) 2539

Zellmorphologie und Prognose der Paraleukoblastosenleukämie ( $\mathrm{M}$ u n d, J., J a c o b i, H., K ü nze r, W.) 2550

Das IgA- und IgG-System bei spastischer Bronchitis im Kindesalter ( $\mathrm{T}$ ü $\mathrm{r} \mathrm{k}, \mathrm{E}$., W i e r s b i tzk y, S.) 2554

Der Wilms-Tumor im ersten Lebensjahr. Insbesondere über 62 Nephroblastome des Neugeborenen (B a c h m a n n, K. D., $\mathrm{K}$. öll, W.) 2598

Bestimmung der Bilirubinkonzentration im Fruchtwasser bei Rh-Inkompatibilität (Reil, B., Schellong, G., Mast, H.) 2602

\section{Laboratoriumsmethoden}

Mikrochemische Urineisenbestimmung bei Säuglingen und Kindern. Untersuchungen zur Urineisenbestimmung vor und nach Deferoxamin-Gabe bei Gesunden, Patienten mit Eisenmangel und Eisenüberladung (M a a s, B., Neth, R., Gos chenh of e r, D., S c hä fe r, K. H.) 1410

Die diagnostische Wertigkeit des Trijodthyronin-Tests (S c r i b a , P. C.) 1455 A. D.

Xyloseresorption in Beziehung zu Leberfunktion und Ernährung bei chronischem Alkoholismus (I r s i g l e r, K., P o in t n e r, H., Krys pin - Exner, K.) 1532

Schwerwiegende Fehlbeurteilungen bei der Urinuntersuchung auf Glucose mit »Schnelltesten « (O l b i n g, H., W e $11 \mathrm{~m}$ a n n, P.) 1566

Magensaftuntersuchung (D e m ling, 1666
Die Bedeutung des $\mathrm{K}^{+} / \mathrm{Ca}^{++}$-Quotienten sowie des isolierten Kalium-Erhöhung im Liquor cerebrospinalis für die Beurteilung zentralnervöser Funktionen ( $\mathrm{Pri}$ il, A.) 1743

Neue Normalwerte für die Lungenfunktionsprüfung mit der Ganzkörperplethysmographie (Amrein, R., Keller, R., Joos, H., H e r zog, H.) 1785

Die Raster-Elektronenmikroskopie (R e u $\mathrm{mut} \mathrm{h}, \mathrm{H}$.) 1832 Ú.

Verzögerte Reaktion von Morbus HodgkinLymphozyten auf Phytohämagglutinin (H a ve m a n n, K.) 1867

Die klinische Bedeutung der Lactatdehydrogenase (E i s e n b u r g, J.) 1882 A. D.

Die je-Glutamyl-Transpeptidase-Aktivität im Serum bei hepatobiliären Erkrankungen (Szasz, G., Rosenthal, P., Fritzsche, W.) 1911

Komplementbindende Antikörper gegen das Zytomegalievirus bei Kindern und Erwachsenen (P e ll e r, P., G o e t z, O.) 1966

Blutsenkungsreaktion bei Leberkrankheiten (F r a n k e n, F. H.) 2007

Lipase und Amylase im Serum bei Stimulation des normalen und geschädigten Pankreas mit Sekretin-Pankreozynin ( $\mathrm{G}$ o e b e 11, H., B od e, Ch., L e m b e r g, G.) 2086

Die Neuraminidase als pathogenetischer Faktor bei Pneumokokken-Infektionen ( $\mathrm{M}$ ü lle r, H. E.) 2149

Erfahrungen mit einer einfachen Methode zur quantitativen Keimbestimmung aus dem Urin (W ill e, L., W in t e r, J.) 2223

Geringer Aktivitätsanstieg der Serum-Transaminasen ( $\mathrm{S} \mathrm{ch} \mathrm{m} \mathrm{i} \mathrm{d} \mathrm{t,} \mathrm{F.} \mathrm{W.)} 2250$

Plasma-Renin bei akuter und chronischer Niereninsuffizienz und Hämodialyse (S chröder, E., Herms, W., We t z els, E., Dume, Th., Grabense e, B.) 2262

Die Endoradiosonde als pH-Indikator bei der endogastralen Titration (D e y h le, P., $\mathrm{M}$ i ed e rer, S. E., S t a d e lmann, O.) 2274

Elektrophoretische und immunologische $\mathrm{Be}$ funde beim Marfan-Syndrom (S u s c h k e, J., Heilmann, K., Murken, J.-D., S t e h r, K.) 2289

Xyloseresorption bei chronischem Alkoholismus (Irsigler, K., Pointer, H., Krypspin-Exner, K.) 2297

Orthostatische Blutdrucklabilität beim Phäochromozytom (W e r n in g, C.) 2299

Das reversible procainamidinduzierte Lupuserythematodes-Syndrom ( $G$ ü $n t h$ e r, R., A s a m e r, H., D it tri c h, P.) 2338

Zur biologischen Aktivität pathogener Staphylokokken (J e l ja sze w i c z, J., Pulve r e r, G.) 2349 U.

Freies Thyroxin und proteingebundenes Jod im Serum bei Eu- und Hyperthyreose (K r üs ke m per, H. L., Her r man n, J.) 2386

Porphyrine im Magen- und Duodenalsaft (D o s s, M., Filip pin i, L.) 2392

Glucose-Assimilationskoeffizient ( $\mathrm{K} \ddot{\mathrm{u}} \mathrm{h} \mathrm{n}$ a $\mathrm{u}$, J.) 2458

Bakteriologische Untersuchungen mit Carbenicillin (B I a s i u s, C h., M e n z, H.-P., S a n d e r, J.) 2501

Zur Antibiotikatherapie der Nocardiose (S c ha a l, K. P., Le is chi k, W.) 2505 Thrombotest und Quick-Test (Voss, D.) 2525

Laboratoriumsbefunde bei Paraproteinämien (K l e m m, D.) $2558 \mathrm{~A}$. D.
Zur Bestimmung von Glomerulumfiltrat und effektivem renalem Plasmafluß. Prüfung eines vereinfachten Einmalinjektionsierfahrens (We i d m a n n, P., Fis cher, W. M üller-Duysing, W., Horst, W., Schönbeck, M., Werning, C. $\mathrm{H}$ ül s, W., S i e ge n th a le r, W.) 2685

\section{Mund, Hals, Nase, Ohren}

Cortison-Dauertherapie trotz Zahngranulom? (K a i s e r, H.) 1467

Endogener idiohypophysärer Diabetes mellitus (W a ld d äusl, W., B u ri a n, K., B e r ing e r, A.) 2028

Periodisch auftretende Aphthen (Solter m a n n, W.) 2121 L. Z

\section{Nervensystem und Psychiatrie}

Interne Liquordrainage bei Hirntumoren mit Hydrozephalus (D r i e s e n, W., To dorow, S.) 1418 A. Th.

Fernsehschäden bei Kindern (Uld a 11, B.) 1453

Legasthenie und Ganzwortmethode (I s l a r, K.-G.) 1462 L. Z.

Sexualstörungen durch Valium (C o p e r , H.) 1467

Funktionspsychosen als Manifestationen der $\mathrm{B}_{1 \text { - }}$-Avitaminosen (W i e c k, H. H., P r i billa, W., Heerklotz, B.) 1473

Zur Fieber- und Penicillinbehandlung der progressiven Paralyse in neuropathologischer Sicht ( $\mathrm{Q}$ u a n d t, J., S o m mer, H.) 1486

Die Bedeutung der Mikrochirurgie in der Hirnchirurgie (Y a s a rgil, M. G.) 1496 A. Th.

Perivenöse Markdestruktionen bei eitriger Meningitis ( $\mathrm{St}$ a m m le r, A.) 1529

Xyloseresorption in Beziehung zu Leberfunktion und Ernährung bei chronischem Alkoholismus (I rsigler, K., P o in te r, $\mathrm{H}$. Kryspin-Exner, K.) 1532

Alkohol und Leberschaden (M e yer $\mathrm{z} \mathrm{u}$

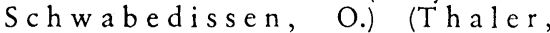
H.) 1544 L. Z.

Schmerzen bei Amputationsneurom ( $\mathrm{M} \mathrm{u}$ $\mathrm{menth}$ a le r, M.) 1585

Die Bedeutung der Mumpsmeningitis ( $\mathrm{R} \mathrm{adl}$, H.) 1599

Der Hydrozephalus des Erwachsenen (B e $\mathrm{n}$ in i, A.) 1617 A. D.

Die Bedeutung des $\mathrm{K}^{+} / \mathrm{Ca}^{++}$-Quotienten sowie der isolierten Kalium-Erhöhung im Liquor cerebrospinalis für die Beurteilung zentralnervöser Funktionen (P r i ll, A.) 1743

Segmentale motorische Paresen beim Zoster (Schli ack, H., Schneider, H.) 1861

Odem nach Antiepileptika (Schuh, F.) 1897

Ursachen medikamentös bedingter parkinsonähnlicher Symptome (J u r n a, I.) 1941 Ư.

Funktionspsychosen als Manifestationen der $\mathrm{B}_{12}$-Avitaminosen ( $\mathrm{R}$ i n g w a l d, E.) 1945 L. Z.

Neurologische Erkrankungen durch Enteroviren bei Kindern (W indorfer, A. S it z m a n n, F. C.) 2071

Paraneoplastische Polyneuropathie bei Thymuskarzinom ( $\mathrm{M} \mathrm{i} \mathrm{el} \mathrm{k} \mathrm{e,} \mathrm{F.,} \mathrm{W} \mathrm{ol} \mathrm{te} \mathrm{r,}$ M.) 2102 
Zur Problematik des dissoziierten Hirntodes bei Patienten eines internistischen Intensivpflegezentrums (Schuster, $\quad H$. P., B us ch, H., B u s ch, G., Wi e m c z y k, H., B a um, P., Knolle, J., Frh. v. Ung e r - S t e rn ber g, A., Lang, K.) $2118 \mathrm{~K}$.

Lungenembolie und symptomatische Psychose (R i e d e r e r, J.) 2181 L. Z.

Hirndurchblutung bei Digitalistherapie (Go t t s t e in, U.) 2182

Differentialdiagnostische Probleme bei Tuberkulomen des Gehirns ( $\mathrm{M}$ öbi us, W.) 2281

Xyloseresorption bei chronischem Alkoholismus (Irsigler, K., P o in tner, H., Krys p in-Exner, K.) 2297 L. Z.

Suchtgefahr bei Distraneurin ( $\mathrm{H}$ u f f m a n n, G.) 2299

Moschcowitz-Syndrom. Ein Beitrag zur »thrombotisch thrombozytopenischen Purpura " mit gerinnungsphysiologischen und pathologisch-anatomischen Untersuchungen (L e chle r, E., Kle in, H. O., Sch u m a cher, K., Gross, R., Eder, M., K un s t, H.) 2326

Phantome nach Amputation und bei angeborenem Gliedmaßenmangel ( $\mathrm{P}$ o e c k, K.) 2367

Zur Feststellung des Hirntodes ( $\mathrm{S} \mathrm{ch}$ ne i d e r, H.) 2405 L. Z.

Wurzelreizerscheinungen nach Diuretika? (J a n z e n, R.) 2408

Zur Therapie der zerebralen Insuffizienz (Grill, P., B ro i c he r, H.) 2429

Behandlungsmöglichkeiten bei Hypophysentumoren (W e b e r, G.) 2442 A. Th.

Sepsis und Meningitis durch Vibrio fetus (Stille, W. Helm, E. B.) 2484

Zum gegenwärtigen Stand der Lithium-Therapie (L a u t e r, H.) 2512 Ü.

Oligophrenie mit wahrscheinlich geschlechtsgebunden-rezessiver Vererbung ( $\mathrm{Ne} \mathrm{u}$ hä us e r, G., Z e rb in - R üd i n, E.) 2519

Virusinfektionen und Parkinson-Syndrom (U m b a ch, W.) 2525

Wadenschmerzen (M ülle r, N.) 2525

Behandlung mit $\beta$-Rezeptorenblockern ( $L$ y d t in, H.) $2560 \mathrm{~A}$. Th.

Therapie zerebralsklerotischer Verwirrtheitszustände (R ü m m e l e,W.) 2571

Achtzig Jahre Uncinatus-Anfälle (W o r m se r, P.) 2618

Lithiumtherapie bei Psychosen (L a uter, H.) 2659

Uber X-chromosomale Zahlabweichungen, insbesondere Mosaike bei Anstaltspatienten (Hessing, J., Kabarity, A., S c h a d e, H.) 2675

Behandlung des Morbus Parkinson mit L-Dopa in Kombination mit einem Decarboxylasehemmer (S i e g frie d, J., Kla i ber, R., Perret, E., Ziegler, W. H.) 2678

Der Vena-subclavia Katheter (Volles, E., Gressner, P., Dahlmann, W., Prill, A., S a bun c u, N.) 2682

Chorea major: prophylaktische Behandlung? (P o e c k, A.) 2703

\section{Pathologische Anatomie und Allgemeine Pathologie}

Indikationen zur Bestimmung der Reninaktivität im Blut (Hod l e r, J.) 1417 A. D.

Úber primäre Sarkome des Hauptstammes der Arteria pulmonalis ( $\mathrm{C}$ a i n, H.) 1607
Beitrag der Nierenbiopsie zum nephrotischen Syndrom (B o hle, A., Kluthe, R., Sarre, H., W e h ne r, H.) 1673

Suizid durch Genuß von Bananen (R i t $t, E$, A n d r a s s y, K., Z i e gle r, M., Kremp i e n, B.) 1767 L. Z.

Das Goodpasture-Syndrom. Ein klinischer und pathologisch-anatomischer Beitrag (Krauss, J., Redenbacher, M. Streicher, E.) 1820

Die Raster-Elektronenmikroskopie ( $\mathrm{R} \mathrm{e} \mathrm{u} \mathrm{-}$ m u t h, H.) 1832

Die kleinherdige hypoxidotische Herzmuskelnekrose (P o c h e, R.) 1851

Angeborene pulmonale Lymphangiektasien. Bericht über 4 Fälle ( $\mathrm{Gl}$ e i s s ner, $P$. A s a n t e, F., S chubert, G. E.) 1987

Paraneoplastische Polyneuropathie bei Thymuskarzinom (M i elk e, F., W ol te r, M.) 2102

Intermittierender Verschluß der Arteria poplitea durch zystische Adventitiadegeneration (E h r ing e r, H., D e n c k, H., W u ke t ich, St., B runner, E.) 2107

Korrelation des laparoskopischen und histologischen Befundes bei chronischer Hepatitis und Leberzirrhose (Solterma n W.) $2121 \mathrm{~L} . \mathrm{Z}$.

Autonomer Hyperparathyreoidismus infolge Nebenschilddrüsenadenom nach Nierentransplantation (Kistler, H. J., Scheit lin, W., Mayor, G., Uehlinger, E.) 2140

Erfolgreiche Operation eines Pseudotumors nach Oberschenkelfraktur bei Hämophilie B. Zugleich ein Beitrag zur Plasmapherese (F i s che r, M., Fuchsig, P., Lechner, K., Pilgerstorfer, H. W. Salzer, Mechthild, Steinbere it h n e r, K.) 2145

Die Diagnose des senilen Herzamyloids (S t e in m a n n, B.) $2170 \mathrm{~A}$. D.

Struktur und Funktion des juxtaglomerulären Apparates der Niere unter geordneten $\mathrm{Be}$ dingungen ( $\mathrm{C}$ a i n, H., $\mathrm{K} \mathrm{r}$ a u s , B.) 2173 Ư.

Die derzeitige Häufigkeit der einzelnen Lymphknotenerkrankungen in SchleswigHolstein (L e n n e r t, K.) 2194

Zum Problem der sogenannten Transplantationslunge ( $\mathrm{B} \mathrm{e} \mathrm{c} \mathrm{k} \mathrm{e} \mathrm{r,} \mathrm{H.} \mathrm{D.,} \mathrm{H} \ddot{u} \mathrm{~b}$ n e r, G., E i gle r, F. W., S i e b e rt, H. G.) 2202

Angeborenes adrenogenitales Salzverlustsyndrom mit Entfaltung einer Prostata bein weiblichen Säugling ( $\mathrm{c} \mathrm{h} \mathrm{u} \mathrm{h} \mathrm{m} \mathrm{a} \mathrm{n} \mathrm{n,} \mathrm{R.)}$ 2229

Pseudo-Pelger-Zellen vom homozygoten Typ bei unreifzelliger myeloischer Leukämie (Hennekeuser, H.-H., Fischer, R., T a l k e, H., M a in zer, K.) 2284

Moschcowitz-Syndrom. Ein Beitrag zur »thrombotisch thrombozytopenischen Purpura « mit gerinnungsphysiologischen und pathologisch-anatomischen Untersuchungen (L e chler, E., Kle in, H. O., Schum a cher, K., Gross, R., Eder, M., Kunst, H.) 2326

Zur Pathologie der Osteopathie nach Heparinbehandlung ( $\mathrm{S}$ ch u s t e r, J., M e i e r. R u g e, W., E g li , F.) 2334

Uber eine Familie mit Goodpasture-Syndrom, Alport-Syndrom und Asthma bronchiale (M ü n t ef e ring, H., Hils cher, W., $\mathrm{M}$ i e s e, A.) 2637

\section{Arzt und Recht}

Angestellte des vertrauensärztlichen Dienstes und Schweigepflicht (Kohlh a s, M.) 1423

Zur Aufklärung bei perkutaner Leberbiopsie (Kohlha a s, M.) 1463

Zur Weitergabe ärztlicher Befunde an fremde Personen oder Patienten ( $\mathrm{K}$ oh lh a a s, M.) 1503

Begrenzung des Anweisungsrechtes gegenüber beamteten Chefärzten (G rö mig, U.) 1544

Nochmals: Trunksucht als Krankheit (Kohl$\mathrm{h}$ a a s, M.) 1624

Neue Steuerrechtsprechung zu den Auslandsreisen für Fortbildungszwecke (W u t h , K.) 1704

Ärztlicher Fachkongreß und Persönlichkeitsbildung (W u th, K.) 1704

Meldung von Krankheiten an die Gesundheitsämter (Ko h l h a a s, M.) 1768

Zur Herausgabepflicht hinsichtlich der Unterlagen beschuldigter Ärzte (Kohlh a a s. M.) 1768

Zur Haftung für Druckfehler in medizinischen Veröffentlichungen (Kohlh a as, M.) 1839

Ersatzanspruch bei Arbeitsvertragsbruch der Arzthelferin ( $\mathrm{R}$ i e g e r, H.-J.) 1840

Zur Haftung des Arztes für Fehler des Hilfspersonals (B r a n d t, H.) 1946

Rückgabe entliehener Arztunterlagen ( $\mathrm{R}$ i e g e r, H.-J.) 1947

Nochmals: Arzt und Transfusionsverweigerer (Koh l h a a s, M.) 2005

Rechtsfragen zum ärztlichen Notdienst aus ärztlicher Sicht (S p o n h e i m e r, K.) 2005

Arztgeheimnis und Schutz der Menschenwïrde (Ko hl h a a s, M.) 2060

Honorierung bei Mißbrauch des ärztlichen Notfalldienstes ( $\mathrm{Pa} \mathrm{s} \mathrm{c} \mathrm{h} \mathrm{k} \mathrm{e,} \mathrm{R.)} 2061$

Arzt und Operationsverweigerer (Koh l$h$ a a s, M.) 2122

Zur Haftung des Arztes für Fehler des Hilfspersonals ( $\mathrm{F} \mathrm{r}$ i e d, K. H.) 2240

Auskunftspflicht des Privatversicherers (Kohlh a as, M.) 2242

Begutachtung durch Medizinalassistenten und ausländische Ärzte (Kohlh a s, M.) 2243

Der Kostenaufwand für Arzneimittel und Diät bei der Einkommenssteuer ( $\mathrm{W}$ u t h , K.) 2243

Nochmals: Zum Nacht- und Bereitschaftsdienst in Kliniken (Kohlhaas, M.) 2298

Wer ist über Krankenunterlagen verfügungsberechtigt? (K o h $1 \mathrm{~h}$ a a s, M.) 2357

Die ärztliche Gutachtertätigkeit bei der Umsatzsteuer (W u t h, K.) 2454

Schritte zur Kostensicherung (Kohlh a a s, M.) 2523

Die Anzeigepflicht des Leichenbeschauers (Kohlha as, M.) 2570

Rechtsfragen des Belegarztes bei der Umstellung vom kleinen auf den großen Pflegesatz (K r äm e r-G ün th e r, H.) 2616

Zur Haftung des Arztes für Fehler des Hilfspersonals (Koh $1 \mathrm{~h}$ a a s, M.) 2656

Zur Regreßpflicht des Amtsarztes (R i e g e r, H.-J.) 2656

\section{Standes- und Berufsangelegenheiten}

Zur Erleichterung der Fortbildung (B e ck e r, J.) 1543 L. Z.

Ärztliche Gebühren bei Privatpatienten (B e r e n s m a n n, R.) 2526 


\section{Strahlenkunde}

Echinacin-Test zur Leukozytenprovokation bei effektiver Strahlentherapie (C h o né, B., $\mathrm{M}$ a n i d a k i s, G.) 1406

Leukämie durch Röntgenstrahlen (Fli ed $\mathrm{n}$ e r, T. M., A d a m, W.) 1426

Elektronische Verbesserung von Röntgenaufnahmen (R o th, F.-J., Wenz, W., $\mathrm{Kr}$ a m e r, H.) 1483

Röntgentherapie des Morbus Bechterew (D i e h $1 \mathrm{~m}$ a n n, W.) 1505

Tonus und Mobilität von Magen und Zwölffingerdarm nach selektiver Vagotomie und Pyloroplastik (D a h m, K., S a p o u n o v, S.) 1513

Erfahrungen mit der Leberszintigraphie (U m e k, H., C z e m b i r e k, H.) 1574

Katarakt durch Röntgenstrahlen (K r o k ow $\mathrm{s} \mathrm{k} \mathrm{i,} \mathrm{E.)} 1585$

Nierendurchblutung und Glomerulumfiltrat unter Dihydralazin-Wirkung (W ü r d i n ge r, H., A u s t, W., M üll e r, U.) 1688

Gibt es charakteristische Röntgenbefunde bei der Gicht? (D i l lmann, W., Fernh ol z, H. J.) 1909

Pharmakoangiographie des Pankreas mit Sekretin und Adrenalin ( $\mathrm{C}$ e $\mathrm{n}, \mathrm{M}$., $\mathrm{R}$ o s e n b u s ch, G., Frik, W., K a I f f, G.) 1970

Zum diagnostischen Wert der Leberszintigraphie (B e r n d t , H.) 2003 L. Z.

Die Effektivität einer präventiven Krebsbekämpfung (O e s e r, H., R a c h, K.) 2015

Zur Problernatik der Infusions-CholezystoCholangiographie (Meyer-B u rg, J.) 2018

Das Lungenmycetom und seine Differentialdiagnose (R e i n h a r d t , K.) 2045

Röntgenreihenuntersuchung in der Schwangerschaft ( $\mathrm{N}$ e u m a $\mathrm{n} n$, G.) 2127

Erhöht vorsorgliche Röntgenreihenuntersuchung die Uberlebensrate beim Lungenkrebs? (O e s e r, H.) (B e r n d t , H.) 2180 L. Z.

Röntgenologische Lungenbefunde bei septischen Erkrankungen (D u n k e r, S. Stille, W.) 2226

Freies Thyroxin und proteingebundenes Jod im Serum bei Eu- und Hyperthyreose (Krüskemper, H. L., Herman n, J.) 2386

Indikationen, Möglichkeiten und Grenzen der selektiven Oberbaucharteriographie (P r e ter, B., Wirth, W.) 2395 A. D.

Thorium-X-Behandlung beim Morbus Bechterew (H a i k e, H. J.) 2459

Kontrastmittelspeicherung in der Leber nach Fußlymphographie (Hepatographie) ( $\mathrm{T}$ is m e r, R., Fried ma n n, G.) 2547

Uber das Hyperthyreose-Risiko bei iatrogener Jodzufuhr (S c h n e id e r, C., S t e p h a n, G., S u w e l a c k, M.) 2631

Zur Bestimmung von Glomerulumfiltrat und effektivem renalem Plasmafluß. Prüfung eines vereinfachten Einmalinjektionsverfahrens (W e i d m a n $n, P ., F$ is ch e $r$, W. Mülle r - D u ys ing, W., Horst, W., S chönbeck, M., Werning, C., $\mathrm{H} \ddot{\mathrm{u}} \mathrm{ls}$, W., S i e genth a ler, W.) 2685

\section{Verdauungs- und \\ Stoffwechselkrankheiten}

Glykogenose Typ II (Pompesche Krankheit) mit $\alpha$-Amylase- und Hyaluronidase-Mangel (P l a t t, D., P l a t t, M.) 1414

Indikationen zur Bestimmung der Reninaktivität im Blut (H o d l e r, J.) $1417 \mathrm{~A}$. D.
Biochemie und Klinik der Glykogenosen (P l a t t, D.) 1420 U.

Die hypokaliämische metabolische Alkalose (E u chenhofer, M., Streicher, E., Würz, H., Me urer, K. A., Stei n e r, B., D ü r r, F., K a u f $m$ a n n, W.) 1441

Die Bedeutung enteralen Lysozyms für den Säugling (B r a u n, O. H.) 1458

Zur Aufklärung bei perkutaner Leberbiopsie (Koh Ih a a s, M.) 1463

Leberdiagnostik (D e m li n g , L.) 1466

Die akute Osophagusvarizenblutung. Ein Aufruf zu aktiver Therapie (E s s e r, G., G ü t ge $\mathrm{m}$ a $\mathrm{n} n$, A.) 1476

Arteriomesenteriale Duodenalkompression ( $\mathrm{H} \mathrm{a}$ f t e r, E.) 1495 A. D.

Eisenresorption bei Achlorhydrie ( $\mathrm{M}$ o n t z, R.) 1503 L. Z.

Chronischer Gebrauch salinischer Abführmittel (B i e g e r, D.) 1506

Tonus und Motilität von Magen und Zwölffingerdarm nach selektiver Vagotomie und Pyloroplastik (D a h m, K., S a p o u n o v, S.) 1513

Xyloseresorption in Beziehung zu Leberfunktion und Ernährung bei chronischem Alkoholismus (I rsig le r, K., P o in tner, H., Krys p in - Ex n e r, K.) 1532

Alkohol und Leberschaden ( $M$ e ye $r$ z u

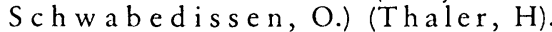
1544 L. Z.

Enterogene Hypocholesterinämie (Kahlke, W.) 1545

Möglichkeiten und Grenzen hochdosierter Diuretikatherapie bei hydropischer Niereninsuffizienz ( $\mathrm{He}$ i d l a n d, A., K l ü t s c h, A., Moormann, A., Hennemann, H.) 1568

Erfahrungen mit der Leberszintigraphie (U m e k, H., C z e m b i r e k, H.) 1574

Die Diagnose der Porphyria cutanea tarda (Filippini, L., Strohmeyer, G.) 1577 A. D.

Zur esterolytischen Toxogonin-Wirkung (He it ma n n, R., Felgenhauer, K.) 1583 L. Z.

Einfluß einer Glucocorticoidbehandlung auf die Proteinsynthese in Leberpunktaten von lebergesunden Menschen (Richter, E., Clauditz, S., Leinweber, B., $\mathrm{K}$ ü h n, H. A.) 1615

Die Therapie der Fettleber (F i l i p p i n i, L.) $1619 \mathrm{~A}$. Th.

Korrelation des laparoskopischen und histologischen Befundes bei chronischer Hepatitis und Leberzirrhose ( $\mathrm{V} \mathrm{i} \mathrm{d} \mathrm{o,} \mathrm{I.,} \mathrm{W} \mathrm{ild} \mathrm{-}$ h i r t, E.) 1633

Zur Frage der postnekrotischen Narbenleber (V i d o, I., Wild h i r t, E.) 1637

Magensaftuntersuchung (D e m ling, L.) 1666

Vitamin $B_{12}$ bei Leberleiden (D e m l i n g, L.) 1770

Die Behandlung spontaner Hypoglykämie bei einem inoperablen Leberzellkarzinom durch Glucagon (M ü he, E., S ch ricker, K. T., R a it h e 1, D.) 1781

Hepatozellulärer Ikterus nach wiederholter intravenöser Infusion von Fettemulsionen bei Kindern mit ausgedehnter Darmresektion (O l b ing, H., Engels, A.-M., D ö n g e s, K. G.) 1825

Konstitutionelle Faktoren bei Leberzirrhose (W e w a l k a , F.) 1827 A. D.

Therapie des Coma hepaticum ( $\mathrm{M}$ a r $\mathrm{k}$ of $\mathrm{f}$, N.) $1829 \mathrm{~A}$. Th.

Zur Frage der posthepatitischen Leberzirrhose (Wildhirt, E.) (Franken, F. H.) 1838 L. Z.
Hypokaliämiezeichen im pathologischen Elektrokardiogramm ( $\mathrm{He}$ in e c k e r, R.) 1841

Fettleber und Eisenstoffwechsel ( $\mathrm{T}$ h a le r, H., S t r o h m e y e r, G.) 1841

Adiopositas in der Antike (Herter, H.) 1842

Katamnestische Erhebungen bei CoeliakiePatienten (W a g n e r, A., K o l b, H. P.) 1856

Vergleich der enzymatischen Aktivität verschiedener Pankreasfermentpräparate in virto (M ül le r - Wieland, K., Berndt, W.) 1870

Zum Problem der exakten Flüssigkeitsbilanz bei der Peritonealdialyse ( $\mathrm{Kr}$ a m e r, $\mathrm{P}$. Quellhorst, E., Henning, H. V., Scheler, F.) 1873

Die klinische Bedeutung der Lactatdehydrogenase ( $\mathrm{E}$ is e $\mathrm{n}$ b u r g, J.) $1882 \mathrm{~A}$. D.

Osmotherapie bei Intoxikationen ( $\mathrm{Mertz}$, D. P.) 1896

Gibt es charakteristische Röntgenbefunde bei der Gicht? (D ihlmann, W., Fernhol z, H. J.) 1909

Die $\gamma$-Glutamyl-Transpeptidase-Aktivität im Serum bei hepatobiliären Erkrankungen ( $\mathrm{S}$ z a sz, G., R os en thal, P., Fritzsche, W.) 1911

Enterale Hämagloblinresorption ( $\mathrm{K}$ ü n z e r , W.) 1950

Diabetische Enteropathie ( $\mathrm{M}$ eh ne rt, $\mathrm{H}$.) 1950

Pharmakoangiographie des Pankreas mit Sekretin und Adrenalin ( $\mathrm{C}$ e $\mathrm{n}, \mathrm{M}$., R ose n bus ch, G., Frik, W., K a Iff, G.) 1970

Zum diagnostischen Wert der Leberszintigraphie (B e r ndt, H.) 2003 L. Z.

Blutsenkungsreaktion bei Leberkrankheiten (F r a n k e n, F. H.) 2007

Zur Problematik der Infusions-CholezystoCholangiographie (M e yer-Burg, J.) 2018

Malabsorptionssyndrom bei partiellem Immunglobulinmangel (IgA-Defekt) (W a g ner, A., Grossma n n, H. D.) 2023

Rezidivierende Polychondritis mit Aortenaneurysmen. Jaksch-Wartenhorst-v.-Meyenburg-Altherr-Uehlinger-Syndrom ( $\mathrm{L}$ a n $\mathrm{g}$, H. D., M üll e r, D., F in ke, J.) 2033

Zum Problem der exakten Flüssigkeitsbilanz. bei der Peritonealdialyse (Engels ing, B.) 2059

Magensaftuntersuchung (D o b r ow ols ki, L.) 2060 L. Z.

Octapressin bei Ösophagusvarizenblutung (H a e m m e rli, U.P.) 2063

Lipase und Amylase im Serum bei Stimulation des normalen und geschädigten Pankreas mit Sekretin-Pankreozymin (G o ebell, H., B od e, Ch., L e m b e r g, G.) 2086

Zur Klinik der Dünndarmdivertikel (W a g n e r, A.) 2097

Indikation zur direkten Cholezysto-Cholangiographie und Cholangiographic (W a n n a g a t, L.) 2111 A. D.

Korrelation des laparoskopischen und histologischen Befundes bei chronischer Hepatitis und Leberzirrhose $(\mathrm{S}$ ol terman , W.) 2121 L. Z.

Toxische Hypercalcämie und reversible Weichteilverkalkungen in der Polyuriephase nach akutem Nierenversagen (L a n ge, H., G o s s m a n n, H. H., S e y b o ld, D., Vog t, O., P f a b , R., D o mbrowski, H.) 2155

Myelofibrose und Osophagusvarizen (Wo h le n berg, H.)

Leberzirrhose nach Magenresektionen, bei Magen-Duodenalgeschwüren und $\mathrm{Ge}$ schwürsnarben ( $\mathrm{M}$ ö r l, H., F e i g e, G.) 2167 
Asystolischer Herzstillstand bei Achalasie (Rösch, W., Bachmann, K., Ottenjan n, R.) 2191

Uber katamnestische Untersuchungen bei jugendlichen Coeliakie-Patienten. Ein Bei trag zur Pathologie des Jugendalters (W e b e r, A., Ri e cken, E. O., Mar t in i, G. A.) 2206

Zur Prognose der chronischen Hepatitis. I. Formen und Entwicklungsstadien ( $\mathrm{V}$ i d o, I., S e l ma ir, H., Wild hirt, E., Ort mans, H.) 2215

Zur Prognose der chronischen Hepatitis. II. Verlaufsdauer (S e l m a i r, H., V id o, I., Wild h i r t, E.) 2220

Möglichkeiten und Grenzen der modernen Leberkoma-Therapie. Austauschtransfusion, Leberperfusion und Leberersatz (Ko m m e r e ll, B.) 2235

Geringer Aktivitätsanstieg der Serum-Transaminasen ( $\mathrm{S} \mathrm{ch} \mathrm{m} \mathrm{id} \mathrm{t,} \mathrm{F.} \mathrm{W.)} 2250$

Die Endoradiosonde als $\mathrm{pH}$-Indikator bei der endogastralen Titration (D e y h le, P., $\mathrm{M}$ i eder er, S. E., S t a de $1 \mathrm{~m}$ an $\mathrm{n}, \mathrm{O}$.) 2274

Der Wert des submaximalen Betazoltests für die Indikation bionomer nichtresezierender Operationsverfahren beim Magen- und Zwölffingerdarmgeschwür ( $R$ e h n e r, M., K o c h, W.) 2278

Diättherapie der essentiellen Hyperlipämien (Gries, F. A., Jahnke, K., Preis s, H., C a nzler, H., M is s, H. D.) 2307

Allgemeine Grundlagen der Diagnostik von Magen-Frühkarzinomen mit der Gastrokamera (O s h i m a, H.) $2440 \mathrm{~A}$. D.

Pollenallergie durch Honig ( $\mathrm{K}$ ü s te r, F.) 2458

Glucose-Assimilationskoeffizient ( $\mathrm{K} \ddot{\mathrm{h}} \mathrm{n} \mathrm{au}$, J.) 2458

Carbenoxolon-Natrium ( $\mathrm{O} t \mathrm{t}$ e $\mathrm{n}$ j a $\mathrm{n} n, \mathrm{R}$.) 2566
Beitrag zum renalen Kaliumverlustsyndrom. Bericht über einen Fall von geleugnetem Diuretika-Abusus (A dolphs, H.-D., I r m s ch e r, K., S o l b a c h, H. G.) 2634 Die Wirkung von Cumarin-, Indandion- und Benzofuran-Derivaten auf die renale Harnsäureausscheidung (Z öllner, N., Gröbner, W.) $2652 \mathrm{~K}$.

Therapie der Amyloidosen ( $\mathrm{M}$ is s mah $\mathrm{l}$, H. P.) 2691 A. Th.

Das aktive Zentrum von Enzymen ( $\mathrm{K}$ i s $\mathrm{ch}$, K.) 2693 U.

\section{Verschiedenes}

Sichtverbesserung durch gelbe Blendschutzbrille? (S c h o b e r, H.) 1545

Das Problem der Krebserkrankungen in der Vorstellung der Bevölkerung ( $\mathrm{Neum}$ a n n, G.) $1581 \mathrm{~K}$.

Hitzeberuf bei Nierensteinleiden (Lutze yer, W.) 1666

Nachweis von Kohlenmonoxid zur Diagnostik von CO-Vergiftung ( $\mathrm{Z}$ or $\mathrm{n}, \mathrm{H}$.) 1692

Behandlung des Hitzschlags ( $M$ ü 11 e r, W. A., Kerkhoven, P., Truninger, B.) 1695

Anatomische und technische Möglichkeiten der intravenösen Infusionsbehandlung (S c hult e, H. D.) 1793

Die Raster-Elektronenmikroskopie (R e u m u th, H.) 1832 Ü.

Die paroxysmale nächtliche Hämoglobinurie eine »myeloproliferative Erkrankung«? (M a r ti n, H.) $1891 \mathrm{~K}$

Xyloseresorption bei chronischem Alkoholismus (B e r nd t, H.) 1893 L. Z.

Legasthenie und Ganzwortmethode ( $\mathrm{B}$ a u e $\mathrm{r}$, J.) 1893 L. Z.
Zur Entdeckung der DNS durch Friedrich Miescher vor 100 Jahren ( $\mathrm{H}$ a r b e r s, E.) 1948

Insektizide im Krankenhaus (Hens chler, D.) 2126

Circuit-Training im Schulsport (Hollm a n $n, W)$.

Klimaanlagen in Arbeitsräumen (E y e r , H.) 2251

Uber die »Rückrechnung " aus BlutalkoholAnalysenwerten bei Trunkenheitsdelikten im Verkehr (Elbel, H., Kn ü pling, H.) 2259

Verhütung von Skelettfehlbildungen nach mütterlichem Blutverlust durch Elektrolytersatz (Grote, W.) 2342

Die chronisch obstruktiven Atemwegserkrankungen des Bergmanns ( $\mathrm{R}$ e i chel, G., U $1 \mathrm{~m}$ e r, W. T., B uckup, H., S t e m p e 1, G., We rn e r, U.) 2375

Porphyrine im Magen- und Duodenalsaft (D o s s, M., Fil ip p in i, L.) 2392

Zunehmende Unsitte (O b e s, A. R.) 2453

Oligophrenie mit wahrscheinlich geschlechtsgebunden-rezessiver Vererbung ( $\mathrm{Ne} \mathrm{u}$ häuser, G., Z e rbin-Rüdin, E.) 2519

Zur Koronarreserve des Menschen (Doll, E., K e u l, J.) 2563 U.

Ohne Nährwert (R.) $2615 \mathrm{~K}$.

Die biomedizinische Anwendung der Telemetrie (I r n i ch, W.) 2649

Deutsche Klinik für Diagnostik ( $\mathrm{K}$ r u t of $\mathrm{f}$, L.) 2659

Uber X-chromosomale Zahlabweichungen, insbesondere Mosaike bei Anstaltspatienten (H e s s ing, J., K a b a r i ty, A., $\mathrm{Sch}$ a d e, H.) 2675

Therapie der Amyloidosen ( $\mathrm{M}$ is $\mathrm{s} \mathrm{m}$ a h $\mathrm{l}$, H. P.) 2691 A. Th.

Das aktive Zentrum von Enzymen (Krisch, K.) 2693 U.

\section{Buchbesprechungen}

A hrens, G.: Die Urinanalyse. Praxis der chemischen und mikroskopischen Harnuntersuchung ( $\mathrm{B}$ ü $\mathrm{t} \mathrm{tn}$ e r) 1775

A llert, M.-L., B ressel, M., Sökel a nd, J.: Neurogene Blasenstörungen (Hohenfellner) 2460

Allgöwer, M., Bergentz, S. E., Calne, R. Y., Gruber, U. F.: Progress in Surgery (Kern) 1468

A nderson, W.: Boyds Pathology for the Surgeon (Reiffers cheid) 1708

Andrews, J. R.: The Biology of Human Cancer Radiotherapy (O e s e r) 2573

Angst, J.: Die somatische Therapie der Schizophrenie (M e y e r) 2128

A r nold, M.: Histochemie. Einführung in Grundlagen und Prinzipien der Methoden (S andritter) 2253

A r tz, C. P., H a r d y, J. D.: Complications in Surgery and their Management ( $\mathrm{N}$ is s e n) 2128

A sk-Upmark, E.: A Primer of High Blood Pressure (A r nold) 2705
B a umgart l, F., Kremer, K., Schrei be r, H. W.: Spezielle Chirurgie für die Praxis. Band II, Teil 1: Verdauungssystem (Niss e n) 2064

B e ck, K., D is chle r, W., Helms, M., $\mathrm{K}$ i a n i, B., S i cking er, K., T enner, R.: Atlas der Laparoskopie (A f folter) 2460

B e c k e r, P. E.: Humangenetik. Ein kurzes Handbuch in fünf Bänden. Band I, Teil 2: Merkmale des Gesichtes - Schmecken und Riechen - Merkmale der Gliedmaßen Papillarleisten - Psychologie (F u h r m a n n) 1951

B e cker, W., Buckingham, R. A., Holinger, P. H., Kort ing, G. W., L e d e rer, F. L.: Atlas der Hals-NasenOhren-Krankheiten einschließlich Bronchien und Osophagus (W ulls te in) 2064

B e g em an n, H., Harwerth, H.-G.: Praktische Hämatologie. Differentialdiagnose - Therapie - Methodik (G e h r m a n n) 2661
B e i ckert, A.: Die Glukokortikoid-Therapie innerer Erkrankungen ( $\mathrm{K}$ a i s e r) 1427

B e rde, B.: Handbuch der experimentellen Pharmakologie. Heffter-He ub ner. Vol. XXIII: Neurophypophysial Hormones and Similar Polypetides.(K onzett) 1774

Bergsma, D.: Birth Defects (L a nge) 1628

B e $r \mathrm{nh}$ a rd, S. A.: The Structure and Function of Enzymes ( $\mathrm{Harbers}$ ) 2705

B e s k e, F.: Lehrbuch für Krankenschwestern und Krankenpfleger ( $\mathrm{Her} \mathrm{tl)} 1708$

B e y e r, H.: Lehrbuch der Organischen Chemie (R.) 1587

Bockelmann, P.: Strafrecht des Arztes (Kohlh a a s) 1843

B reddin, K.: Die Thrombozytenfunktion bei hämorrhagischen Diathesen, Thrombosen u. Gefäßkrankheiten (W e i t b r e c h t) 1953

B r e h m, H.: ABC der modernen Empfängnisverhütung ( $\mathrm{D}$ ö r i n g) 1952 
B rothwell, D., Sandison, A. T. Diseases in Antiquity (W e $11 \mathrm{~m}$ a n n) 2185

$\mathrm{B}$ r ü h l, W.: Leber- und Gallenwegserkrankungen ( $\mathrm{K}$ ü h n) 2459

Decher, H.: Die zervikalen Syndrome in der Hals-Nasen-Ohren-Heilkunde ( $\mathrm{Z}$ i m m e r) 2527

Die the lm, L., Olsson, O., Strnad, F., Vie ten, H., Z u p p inger, A. Handbuch der medizinischen Radiologie. Bd. IV, Teil 2: Skeletanatomie $(S \mathrm{t} u \mathrm{tz})$ 2184

Dihlmann, W.: Spondylitis ankylopoetica. Die Bechterewsche Krankheit $(\mathrm{O} t \mathrm{t}) 1586$

D r i s c h e l, H., T i e d t , N.: Biokybernetik, Band I und II ( $B$ ünn ing) 1953

E a s th a m, R. D., J a n c or, J.: Clinical Pathology in Mental Retardation ( $\mathrm{Har}$ b a u e r) 1775

E w e r b e c k, H., H ü t e r, K. A.: Erfassung und Betreuung von Risikofällen während der Schwangerschaft, Geburt und Neugeborenenperiode ( $\mathrm{S}$ t o 11) 2253

F e ls o n, B., We in ste in, A. S., S p it z, H. B.: Röntgenologische Grundlagen der Thoraxdiagnostik (F r i k) 2622

Fourman, P., R o y e r, P.: Calcium Metabolism and the Bone (B a r t e l h e i m e r) 1774

Fre y, E. K., Kra u t, H., W e r le, E.: Das Kallikrein-Kinin-System und seine Inhibitoren (Creutzfeld t) 1668

Gardner, L. I.: Endocrine and Genetic Diseases of Childhood (B l u n c k) 2184

Garré / S t i ch / B a u e r: Lehrbuch der Chirurgie ( $\mathrm{Ke} \mathrm{r} \mathrm{n}$ ) 1547

G ell i s, S., K a g a n, B. M.: Current Pediatric Therapy ( $\mathrm{S}$ c hä f e r) 1507

Gerok, W.: Primäre Tuberkulopathien. Störungen des zellulären Transportes (C a in) 2704

Gillma n n, H.: Physikalische Therapie (D e li u s) 2009

G l a s e r, E. M.: Die physiologischen Grundlagen der Gewöhnung (D a vi d) 2065

Glauner, R., Rüttimann, A., Thurn, P., Via monte, M., Vogler, E.: Ergebnisse der medizinischen Radiologie. Band II: W. B e s s le r: Skeletszintigraphie mit Radiostrontium; E. Z e it l e r : Die Myelographie mit einer neuen Kontrastmittelsuspension; E. Munte an, H. Schreyer, G. Zechner: Der Wert der Schichtuntersuchung bei der Otitis media chronica und der Cholesteatomeiterung; W. B a u e $r$ : Laryngographie (Frik) 2704

Goldstein, A., Aronow, L., Kal $\mathrm{m}$ a $\mathrm{n}$, S. M.: Principles of Drug Action. The Basis of Pharmacology ( $L$ ü $11 \mathrm{~m}$ a n n) 1952

Gould, S. E.: Pathology of the Heart and Blood Vessels ( $\mathrm{S} \mathrm{ch}$ o e $\mathrm{n} \mathrm{m} \mathrm{a} \mathrm{c} \mathrm{k} \mathrm{e} \mathrm{r} \mathrm{s)} 2622$

Gross, D.: Psychopharmaka und Psychotherapie in Klinik und Praxis (P öl d i nge r) 2185

G s e 11, O.: Antibiotica et Chemotherapie, Vol. 14: Fortschritte - Advances - Progrès (N a u $m$ a n n) 1774

Graciansky, de P., B oulle, S.: Atlas der Dermatologie (He r z b e r g) 1508

Grumbach, A., Kikuth, W.: Die Infektionskrankheiten des Menschen und ihre Erreger ( $\mathrm{Pulv}$ e r e r) 2572

$\mathrm{H}$ a a s e, G.: Physikalische Grundlagen. Physik für Mediziner ( $\mathrm{S} \mathrm{ch}$ e i d) 1548

H a gen, E., We ch sler, W., Zilliken, F.: Experimental Biology and Medicine (R e u t e r) 2527

v o n $\mathrm{H}$ a 11 e r, E.: Gesundheitsbüchlein für die Tropen ( $\mathrm{r}$ r a n z) 2460
Hammersen, F., Gross, D.: Die arteriovenösen Anastomosen. Anatomie, Physiologie, Pathologie, Klinik. Aktuelle Probleme in der Angiologie, Bd. 2 (H e b e r e r) 1952 Hanshaw, J. B., Plowright, W., W e iss, K. E.: Virology Monographs, Vol. 3: Cytomegalieviruses. RinderpestVirus. Lumpy Skin Disease Virus. H o y l e, L.: Vol. 4: The Influenza Viruses Kaplan, AS. Vol. 5: Herpes Simplex and Pseudorabies Viruses (B o n i n) 2662

$\mathrm{H}$ a r p e r, H. A.: Review of Physiological Chemistry (Karls o n) 1586

Hartmann-von Monakow: Therapie der Nervenkrankheiten ( $\mathrm{S}$ c h e i d) 1547

Haynal, I., Matsch, J.: Paroxysmale Tachycardia. Pathogenesis and Treatment of the Supraventricular Form ( $\mathrm{E} f \mathrm{fer}$ ) 2574

$\mathrm{Heg}$ li in, R.: Differentialdiagnose innerer Krankheiten ( $\mathrm{S} \mathrm{ch} \mathrm{ölm} \mathrm{e} \mathrm{r} \mathrm{i} \mathrm{c} \mathrm{h)} 2661$

$\mathrm{H}$ e in $\mathrm{t} z, \mathrm{R}$.: Nieren-Fibel ( $\mathrm{R}$ e u b i) 1843 Herrlinger, R.: Geschichte der medizinischen Abbildung, Band I: Von der Antike bis um 1600 ( $\mathrm{M}$ a n n) 2253

Herrmann, A.: Gefahren bei Operationen an Hals, Ohr und Gesicht und die Korrektur fehlerhafter Eingriffe (B e c k e r) 1586

Hert l, M.: Kinderheilkunde und Kinderkrankenpflege für Schwestern ( $\mathrm{S}$ a cht leben) 2573

$\mathrm{Hertz}$, C. W.: Begutachtung von Lungenfunktionsstörungen ( $\mathrm{M}$ a r x ) 1843

$\mathrm{Hobby}, \mathrm{G}$. L.: Antimicrobial Agents and Chemotherapy ( $\mathrm{N}$ a u m a n n) 2065

Holle, F.: Spezielle Magenchirurgie (Re ifferscheid) 1506

$\mathrm{H}$ oll in g s w o r th, J. W.: Local and Systemic Complications of Rheumatoid Arthritis ( $\mathrm{S}$ ü d h of) 2065

$\mathrm{H}$ o $\mathrm{r}$ à $\mathrm{n}$ y $\mathrm{i}$, J.: Die Entwicklungsanomalien der Bronchialgewebe und ihre klinische $\mathrm{Be}$ deutung ( $\mathrm{S}$ e if e $\mathrm{r}$ ) 2185

Hornbostel, H., Ka ufmann, W., Si e genthaler, W.: Aktuelle Diagnostik - Aktuelle Therapie ( $\mathrm{K}$ a i s e r) 2008

$\mathrm{Hraba}$, T.: Mechanism and Role of Immunological Tolerance ( $G$ ü n th e r) 1508

Hull, R. N., Tyrrell, D. A. J.: The Simian Viruses - Rhinoviruses (E g g e r s) 1843

J a n z, D.: Die Epilepsien (S e l b a c h) 2527

J u cker, E.: Fortschritte der Arzneimittelforschung. Vol. 12 (I p p e n) 1427

$\mathrm{K}$ äh l e r, H. J.: Störwirkungen von Psychopharmaka und Analgetika ( $\mathrm{He}$ in $\mathrm{rich}$ ) 2528

Kä se r, O., Frie dbe r g, V., O be r, K. G., Th o m s e n, K., Z a nd e r, J.: Gynäkologie und Geburtshilfe, Band I: Die geschlechtsspezifischen Funktionen der Frau und ihre Störungen (K i r ch h off) 1627

Kanter, H.: Libyen - Libya. Eine geographisch-medizinische Landeskunde (B e k k e r) 2461

$\mathrm{K}$ a u $\mathrm{f} f \mathrm{~m}$ a $\mathrm{n} n$, F.: Erinnerungen eines Bakteriologen. Zur Geschichte der Enterobacteriaceen-Forschung (W in k le) 1628

Ka z n e r, E., S chief e r, W., Z ül ch, K. J.: Proceeding in Echo-Encephalography. International Symposium (K li n g l e r) 2573

K e m pe, L. G.: Operative Neurosurgery, Bd. 1: Cranial, Cerebral and Intracrania Vascular Disease ( $\mathrm{P}$ i a) 2410

Kle in, E.: Das Testosteron. Die Struma (St ud e r) 2528

K l e p z i g, H.: Herz- und Gefäßkrankheiten (Wolter t) 1628
K l e p z i g, H.: Herz- und Gefäßkrankheiten (Anschü tz) 2704

Kluth e, R.: Fortschritte in der Diätetik bei Nierenkrankheiten (H e i n t z) 2252

$\mathrm{K}$ ra ye n büh 1, H., M a spes, P. E. $S$ w e e t, W. H.: Progress in Neurological Surgery (U m b a c h) 2622

K ü n k e l, H.: Die Periodik der paroxysmalen Dysrhythmie im Elektroenzephalogramm. Zugleich ein methodischer Beitrag zur Analyse biologischer Zeitreihen ( $\mathrm{K} \mathrm{u} \mathrm{g}$ le r) 2573

L a ng e, M.: Orthopädisch-chirurgische Operationslehre (W e ll e r, S.) 2705

L a ng e n, D.: Die gestufte Aktivhypnose (Schulte) 1708

L a ng e n, D.: Psychodiagnostik - Psychotherapie ( $\mathrm{Schult}$ e) 2409

$\mathrm{L}$ a n g e n, P.: Antimetabolite des Nucleinsäure-Stoffwechsels. Biochemische Grund lagen der Wirkung (W il m a n n s) 2065

Le e, J. A., A t kin s o n, R. S.: A Synopsis of Anaesthesia (W i e m e r s) 1468

Le jeune, F, Bunjes, W. E. DeutschEnglisches-Englisch-Deutsches Wörterbuch für Ärzte. Band II: Englisch-Deutsch (W e II m a n n) 2661

V an der Lende, R.: Epidemiology of chronic non-specific lung disease (chronic bronchitis) (Ul m e r) 2129

$\mathrm{L}$ e o $\mathrm{n} \mathrm{h}$ a rd t , H.: Histologie und Zytologie des Menschen ( $\mathrm{M}$ a y e t) 2300

L o o s e, K. E.: Angiographie und ihre Leistungen. Technik - Ergebnisse - Fortschritte (D ü x) 2008

L üb bers, D.-W., Luft, U. C., The ws, G., W i t z l e b, E.: Oxygen Transport in Blood and Tissue. With contributions by numerous different specialists (W e st ph a l) 1707

L ü ders, D.: Lehrbuch für Kinderkrankenschwestern, Bd. I: Das gesunde Kind und theoretischer Teil ( $\mathrm{He} \mathrm{r} \mathrm{tl)} 2461$

$\mathrm{M}$ as se $\mathrm{rm}$ an n, J. H.: Current Psychiatric Therapies, Vol. VIII ( $\mathrm{M}$ e y e r) 1668

$\mathrm{McKusick,V}$. A.: Humangenetik (J ö r gensen) 2410

$\mathrm{Me}$ ade, R. H.: An Introduction to the History of General Surgery ( $\mathrm{Z} u \mathrm{k}$ s c h w e r d t) 2662

Me nde l, D.: A Practice of Cardiac Catheterisation (E f f e r t) 2527

Mills, J. W.: Diagnostic Methods (B ü t t n e r) 1668

Mountcast le, V. B.: Medical Physiology (Franz) 1548

MSD-Manual der Diagnostik und Therapie (R.) 1468

Müller, Ch., Ci ompi, L.: Senile Dementia (B i n i e k) 2185

$\mathrm{N}$ i e meyer, W.: Kleines Praktikum der Audiometrie (B i e s a ls ki) 2252

$\mathrm{N}$ is sen, R., Pfe iffer, K. M.: Zwerchfellhernien. Klinik, Indikation, Chirurgie, Technik (G ü t g e m a n n) 1844

P a p p w or th, M. H.: Human Guinea Pigs (We $11 \mathrm{~m}$ an n) 2528

Pen in, H., Käufer, C.: Der Hirntod. Todeszeitbestimmung bei irreversiblem Funktionsverlust des Gehirns ( $\mathrm{p}$ a $\mathrm{n} \mathrm{n}$ ) 2183

P e p y s, J.: Hypersensitivity Diseases of the Lungs due to Fungi and Organic Dusts (S e elige r) 2461

Petrilowitsch, N.: Charakterstudien (We i tbrecht) 1953

$P f e$ if $f$ e $r$, E.: Handbuch des Diabetes mellitus ( $\mathrm{J} \mathrm{a} \mathrm{k} \mathrm{ob)} 2301$

P i a , H. W.: Ärztliche Ausbildung, Weiterbildung und Fortbildung ( $\mathrm{K} n$ o $\mathrm{th}) 1773$ 
Prech t l, H. F. R., B e in te m a, D. J.: Die neurologische Untersuchung des reifen Neugeborenen ( $\mathrm{Kruse}$ ) 2128

Preston, R. L., M c E w e n, C.: The Surgical Management of Rheumatoid Arthritis (Mittelm e i e r) 2009

$\mathrm{R}$ a a b, W., K le in s orge, H.: Diagnose von Arzneimittelallergien (I p p e n) 2009

$\mathrm{R}$ a $\mathrm{m}$ s e i e r, H.: Die Transplantationsreak tion als zelluläre Immunität (L a r g i a dè r) 1708

Re ploh, H., Otte , H.-J.: Lehrbuch der Medizinischen Mikrobiologie ( $G$ ä r t n e r) 2184

d e R e u ck, A. V. S., Knight, J.: Ciba Foundation Symposium. Cellular Injury (P e ter s) 1548

$\mathrm{R}$ i ch te r, H. E., B e c k m a n n, D.: Herzneurose (K öh l e) 1773

$\mathrm{R}$ i e ger, R., $\mathrm{Mich}$ a e lis, A., Green, M. M.: A Glossary of Genetics and Cytogenetics. Classical and Molecular ( $\mathrm{F}$ u h r m a n n) 2129

Rös s le r, H., H e is t e r, R.: Aktuelle Probleme des Rheumatismus ( $\mathrm{S}$ ü d h of) 1468

R o y e r, P., H a b ib, R., $\mathrm{M}$ a th i e u, H. Nephrologie im Kindesalter ( $L$ i n n e w e h) 2621

$\mathrm{Rubin}$, Ph., C a s a ret $\mathrm{t}, \mathrm{G}$. W.: Clinical Radiation Pathology, Vol. I u. II (B a r t h) 1427

$\mathrm{S}$ a $\mathrm{m} \mathrm{m}$ a $\mathrm{n}, \mathrm{P}$. D.: Nagelerkrankungen (Pfi ster) 1508

Schettle $r$, G.: Innere Medizin ( $\mathrm{O}$ ock) 2128

Sch i e velbein, H.: Nikotin. Pharmakologie und Toxikologie des Tabakrauches (J a n z) 1951

Schle y er, F.: Leitfaden der gerichtlichmedizinischen Blutspuren-Untersuchung (S c h w e r d) 1548

$\mathrm{Schmidt}$, O. P., Günther, W., B o t tke, H.: Das bronchitische Syndrom (B ü h l m a n n) 1468

Schneeweiss, U.: Allgemeine Mikrobiologie ( $\mathrm{S}$ ch i e k) 1628
Schröder, J., I m m i ch, H.: Früherkennung von Krankheiten als methodisches Problem (F r a n k e) 1844

$\mathrm{Sch}$ u c h a rd t, K.: Fortschritte der Kieferund Gesichts-Chirurgie, Band XIII: Die Therapie des Mundhöhlenkarzinoms (S p i e s l) 1668

$\mathrm{Sch}$ uermann, H. $\dagger$, Greither, A., Hor n s t e i n, O.: Krankheiten der Mundschleimhaut und der Lippen ( $\mathrm{S}$ a $1 \mathrm{f} \mathrm{eld}$ ) 1775

Sexualkunde-Atlas (R.) 2574

$\mathrm{Sh}$ a ckelf ord, R. T.: Diagnosis of Surgical Disease, Volumes I, II and III (K r e m e r) 1952

Shikano, S., Shimizu, K.: Atlas of Fluorescence Fundus Angiography (W e s sing) 1587

Si e gfried, J.: Die Parkinsonsche Krankheit und ihre Behandlung ( $\mathrm{Um} \mathrm{bach} 1426$

$\mathrm{S}$ ig g, K.: Varizen. Ulcus cruris und Thrombosen (S choop) 2009

Sne 11, F. M., Sh u $1 \mathrm{~m}$ a n, S., S pencer, R. P., M o o s, C.: Biophysikalische Grundlagen von Struktur und Funktion, eine Einführung für Naturwissenschaftler und Mediziner. Band I: Bausteine und Organisation des biologischen Systems (H a r bers) 2129

Sorkin, E.: The Immune Reponse and its Suppression ( $\mathrm{G}$ ü $\mathrm{n}$ t h e r) 2662

S potnitz, H.: Modern Psychoanalysis of the Schizophrenic Patient ( $\mathrm{M}$ a t us s e k) 2460

S t a e m m le r, H.-J.: Fibel der gynäkologischen Endokrinologie ( $\mathrm{T}$ a u b e r) 2300

$\mathrm{S}$ t a e $\mathrm{m} \mathrm{m}$ l e r, H.-J.: Fibel der gynäkologischen Endokrinologie ( $\mathrm{S} \mathrm{c} \mathrm{h} \mathrm{u} \mathrm{l} \mathrm{t} \mathrm{z} \mathrm{e)} 2621$

S te r n, C.: Grundlagen der Humangenetik (B e c k e r) 2301

S t u t z, L.: Leitfaden der praktischen Desinfektion und Sterilisation (G r ü n) 1427

Sykes, M. K., McNicol, M. W., Campbe 11, E. J. M.: Respiratory Failure (L u tz) 2410
T ils n e r, V.: Erkrankungen des Blutes und der blutbildenden Organe ( $\mathrm{Sch}$ e iff a r th) 1427

Trüb, C. L., D anie Is, J., H a gen, W., Lehmkuhl, H., Pürckhauer, F., Schröder, E., Stralau, J.: Das öffentliche Gesundheitswesen. Band V: Gutachtenwesen. Teil A: Grundlagen. Teil B: Rechtsvorschriften und Erläuterungen (M a n e k e) 2301

V a le nt in e, G. H.: Die Chromosomenstörungen ( $\mathrm{St}$ o e ckeni us) 2622

Vogel, F., Röhrborn, G., Schleier macher, E., Schroeder, T.: Strahlengenetik der Säuger (Fuhrmann) 2183

v an Vos s, H., Thom a s, P.: Medical Thermography (F r is c h k o r n) 1587

W a n n a a t, L.: Leber, Endokrinium und Wasserhaushalt ( $\mathrm{T}$ h a l e r) 1627

W a t s o n, J. D.: Die Doppel-Helix. Ein persönlicher Bericht über die Entdeckung der DNS-Struktur ( $\mathrm{H}$ a r b e r s) 2410

W i d o w, W.: Atlas zur klinischen Diagnostik des Brustdrüsenkrebses (Grop p) 1508

W i e l a n d, O.: 6th International Congress of Clinical Chemistry, Munich 1966. Vol. 1: Clinical Protein Chemistry. Vol. 2: Clinical Enzymology. Vol. 3: Progress in ClinicoChemical Methods. Vol. 4: Advances in Clinico-Biochemical Research. 1968 (B ü t t ne r) 1844

W i n k le r, K.: Dermatologie. Ein Repertorium ( $\mathrm{J}$ ü l i c h) 2461

W ulls te in, H. L.: Operationen zur Verbesserung des Gehörs. Grundlagen und Methoden ( $Z$ öll n e r) 1773

Z d a n s k y, E.: Röntgenpathologie der Lungentuberkulose ( $\mathrm{G}$ ï r i c h) 1952

Z e m p lén y i, T.: Enzyme Biochemistry of the Arterial Wall as related to Atherosclerosis ( $\mathrm{K}$ r ö z) 2129

$\mathrm{Z}$ i o $1 \mathrm{ko}, \mathrm{H}$. U.: Psychische Störungen bei Studenten (Engelm e i e r) 2252

Zöllner, F.: Hals-Nasen-Ohren-Heilkunde ( $\mathrm{N}$ a u m a n n, N. N.) 2183

\section{Verhandlungsberichte}




\section{Sachverzeichnis ${ }^{1}$}

A

AB0-Blutgruppen und Leberzirrhose 2655

Abdomen, akutes bei Mesenterialgefäßverschluß 1938

Abdomenübersichtsaufnahmen, Verbesserung $\mathbf{1 4 8 4}$

Abführmittel, salinische 1506

Abort und Vibrio fetus $\mathbf{2 4 0 0}$

Acetylcholinesterase, Segment 2696

Acetylcholinprobe und Asthma 1805

B-Acetylglucosaminidase und Pompesche Krankheit $\mathbf{1 4 1 4}$

Acetylsalicylsäure, Wirkung 2010

Achalasie, Herzstillstand 2191

Achlorhydrie und Eisenresorption 1503

Acidose bei Herzschock 1753

-, renale und Therapie 1398

ACTH oder Glucorticoide? 1771

Actinomycin, immunsuppressive Therapie $\mathbf{2 4 4 4}$

Acylamidase bei Leukämie 1993

Adam-Preis 19691845

- - 19702011

Adelphan und Blutdruck 2083

Adenom, eosinophiles und Diabetes 2028

Adenome der Hypophyse $\mathbf{2 4 4 2}$

Adenoviren und Impfstoffe 1659

Adhäsion der Blutplättchen $\mathbf{1 7 3 5}$

Adipositas in der Antike 1842

Adrenalin und PankreasAngiographie 1970

- und Phonokardiogramm Diagnostik 1751

Adreno-genitales Salzverlustsyndrom mit Prostata-

entfaltung 2229

Adventitiadegeneration, zystische 2107

Ärzte, ausländische und

Begutachtung 2243

Aescin bei Nierenstein 2382

Äther und Tollwutvirus $\mathbf{1 7 0 1}$

Athylenimine, immunsuppressive

Therapie $\mathbf{2 4 4 4}$

Affektlabilität und Hydergin 2433

Affen als Städter 1776

Affenviren und Impfstoffe $\mathbf{1 6 6 0}$

Aggregation der Blutplättchen 1735

Agammaglobulinämie, $C^{\prime} ; 3$-Gehalt 1980

AHG bei Hämophilie $\mathbf{1 8 8 7}$

-, Konzentrationen bei Hämophilie A $\mathbf{2 5 4 0}$

Akademie für medizinische Fortbildung 2623

Akinesie, Therapie 2678

Akromegalie und Diabetes bei Hypophysèn-Hyperaktivität 2028

Aktivhypnose, gestufte 1708
Aldactone bei Hyperaldosteronismus 2051

Aldesteron und Hyperaldosteronismus $\mathbf{2 0 5 0}$

Aldosteronismus, postoperativer und posttraumatischer 1898"

-, primärer 1442

-, - normokaliämischer 2559

Alkalose, hypokaliämische metabolische 1441, 2634

Alkohol im Blut, Rückrechnung 2259

- und Herz 1759

- und Hyperlipidämien 2308

- und Leberschaden 1543

Alkoholismus, Krankheit? $1624 \mathrm{R}$

- und Xyloseresorption 1532, 1893, 2297

Alkoholresorption, perkutane 2008

Alkoholwattebausch, Wert 2066

Allergie und Carofur 1685

- und Herzbeteiligung 1758

- und Honig 2458

-, mykogene 2508

- und Thymektomie 2062

Allopurinol, Xanthin-Harnsteine 1549

Alport- und GoodpastureSyndrom 2637

Alprenolol 2560

Alter und Demenz 2185"

Altertum, Krankheiten 2185*

Alupent bei Bronchitis 2234

- und CSN-Stimulator 1719

- bei Herzschock 1753

- und Phonokardiogramm Diagnostik 1751

Amantadin bei Grippe $\mathbf{2 5 1 1}$

- bei Parkinsonscher Krankheit 1776

- bei Virusinfektionen 1541

Amilorid bei Hyperaldosteronismus 2052

Aminasin und Tumorwachstum 2706

Aminoglutethimid und Hyperaldosteronismus 2052

Aminosäurespiegel bei Chorea Huntington 2411

Amöben als Enzephalitiserreger 2186

Amphotericin B bei Lungenmykosen 1992

Ampicillin 2608

- bei Keuchhusten 2435

- bei Pyelitis 1939

- und SchwangerschaftsBaktcriurie 1555

Amputation und Phantome 2367

Amputationsneurose, Schmerzen 1585

Amtsarzt und Regreßpflicht $2656 \mathrm{R}$

Amylase in Arzneien 1870

-, Mangel bei Glykogenose Typ II $\mathbf{1 4 1 4}$

- im Serum und Pankreasstimulation 2086
Amylnitrit und Phonokardiographie $\mathbf{1 7 5 0}$

Amyloid des Herzens, seniles 2170

Amyloidosen, Therapie 2691

Amylopektinose 1420

Anämie bei Coeliakie $\mathbf{1 8 5 8}$

- und Hämoglobinurie 1892

-, hämolytische bei MoschcowitzSyndrom 2326

-, immunhämolytische 2447

-, megaloblastische und LDH 1882

,-- Thiamin-bedürftige 2000

- , perniziöse und $B_{1:-2}$

Avitaminosen 1945

-, - und Funktionspsychosen 1475

Anästhesie, Kompendium 1468*

Analgetika und Psychopharmaka 2528:

Analytik, Biochemische und Preisstiftung 2255

Anaphylaxie, Mechanismus 2010

Anastomosen, arteriovenöse 1952

Anatomie der Infusionsbehandlungen 1793

Andersen Glykogenose 1420

Andrologie, Nomenklatur 2530

Aneurysma dissecans und Arterienverschluß 2315

Angina nodularis mit ECHOViren 1961

- pectoris 2646

- -, CarotissinusnervenReizung 1717

- -, Revaskularisation 1903

- -, Rezeptorenblocker 2561

Angiographie, Diagnostik 2008

- der Koronarien 1904

- Subtraktion 1485

Angioma senile 1991

Angiotensin und Hyperaldosteronismus 2050

- und Renin 2177

Angst und Fernsehen bei Kindern 1454

Anna Monika Stiftung 1899

Anstaltspatienten und Chromosomenabweichungen 2675

Anthrakosilikose des Bergmannes 2375

Antibiogramme von Staphylokokken $\mathbf{1 4 4 0}$

Antibiotika bei Bronchitis 2234

- und Chemotherapie 1774

- und Erythematodes-

Auslösung 2041

- Jahrbuch $2065^{*}$

-, neue 2249

- bei Nocardiose 2505

- und Schwangerschaftsbakteriurie 1557

- bei Tierbiß-Pasteurellose 1819

Anti-D-Globulin, Therapie $\mathbf{1 6 5 5}$

Antiepileptika, Ódem 1896
Antifibrinolytika 2542

Antigenstruktur von Staphylokokken 1449

Antihypertonika und Erythematodes 2041

- und Nephrektomie 2083

Antike und Adipositas 1842

Antikoagulaniien und Chinidin 1845

- und Koronarien 2646

- bei Mitralstenose 1424

-, Therapie 2293

Antikörper und Echo-Virusinfektion 2079

- und Immunsuppresiva $\mathbf{2 4 4 4}$

- gegen Pankreaskarzinom 241

- gegen Zytomegalievirus 1967

Antikörpermangelsyndrom und Komplementanalysen $\mathbf{1 9 7 9}$

Antimetaboliten als Immunsuppressiva 2444

- bei Virusinfektion 1540

-, Wirkung 2065

Anti- $\mathrm{Rh}_{0}(\mathrm{D})$-Prophylaxe in der Schwangerschaft 1433

Antithrombin und W/unddehiszenzen 1405

u.1-Antitrypsin-Mangel und Leberzirrhose 1955

Antitrypsin und Pneumokokkenmeningitis $\mathbf{2 1 5 0}$

Anweisungsrecht und Chefärzte 1544

Anzeigepflicht des Leichenbeschaucrs 2570

Aorta, Faszienklappenplastik und Hämolyse 2212

- und Vitamiu D 1879

Aortenaneurysma und Polychondritis 2033

Aortenisthmusstenose, Isthmusplastik 1522

Aortenstenose, subvalvuläre und Diagnose und Therapie 1537, 1539

Aphthen, periodische 2121

Apparat, juxtaglomerulärer $\mathbf{2 1 7 5}$

Appetitzügler 2007

Aptin $\mathbf{2 5 6 0}$

Arbeitsgemeinschaft, kinderkardiologische 2463

Arbeitsräume, Klimaanlagen 2251

Arbeitsvertragsbruch der Arzthelferin $1840 \mathrm{R}$

Arboviren und Impfstoffe 1660

Arbuz, Aktivitäten 1871

Armphantome 2368

Armumfang, Blutdruckmessung 1950

Arrhythmie bei Herzinfarkt $\mathbf{1 6 4 4}$

Arteria poplitea, Adventitiadegeneration 2107

- pulmonalis, Sarkom 1607

Arterienverschluß, akuter und Chirurgie 2315

Arterienwand, Enzymologie 2129

Arteriitis temporalis Horton und Polymyalgia $\mathbf{2 2 3 2}$

\footnotetext{
${ }^{1}$ Dle fettgedruckten Zahlen bedeuten Originalarbeiten, die mit $\cdot$ versehenen Buchbesprechungen. $R=$ Arzt und Recht.
}

Zahlen in Klammern verweisen auf die medizingeschichtlichen Kurztexte. 
Arteriographie des Oberbauches 2395

Arteriosklerose und cerebrale Insuffizienz 2429

- bei Stadt- und Landbevölkerung 2575

- und Thrombose 2662

Arthritis und $C^{\prime}{ }_{3}$-Gehalt 1980

- bei Gicht 1909

- und Immunsuppressiva 2568

-, rheumatoide und AzathioprinSchäden 2268

-, - und Operation 2009*

Arzneimittel und Einkommenssteuer $2243 \mathrm{R}$

- und Herzmuskelnekrosen 1852

Arzneimittelallergien 2009*

Arzneimittelforschung, Fortschritte $1427:$

Arzneimittelwirkungen, Potenzierung 1845

Arzt- und Ausbildung 1773*

-, Belegarzt und Pflegesatz $2616 \mathrm{R}$

- und Briefgeheimnis $1504 \mathrm{R}$

- und Herausgabepflicht von Unterlagen $1768 \mathrm{R}$

- und Hilfspersonal, Haftung 1946 R, 2240

- und Notdienst $2005 \mathrm{R}$

- und Operationsverweigerer $2122 \mathrm{R}$

- und Schweigepflicht $1423 \mathrm{R}$

- und Strafrecht 1843"

- und Transfusionsverweigerer $2005 \mathrm{R}$

Arztgeheimnis und Menschenwürde $2060 \mathrm{R}$

Arzthaftung beim Hilfspersonal $2656 \mathrm{R}$

Arzthelferin, Arbeitsvertragsbruch $1840 \mathrm{R}$

Arztunterlagen, Rückgabe $1947 \mathrm{R}$

Asbestose 2290

L-Asparaginase, 2249

- bei Leukämie 1993

- zur Therapie 1669

Aspergillus fumigatus der Lunge 2046

Assimilationskoeffizient, Glucose 2458

Astawerke, Betriebsjubiläum 2362

Asthma bronchiale, Differenzierung 1802

- - und Goodpasture-Syndrom 2637

- -, mykogene Allergie 2508

- - und Prostaglandin-Aerosol 2462

Asthmatiker und Pilocarpin 1651

Ateminsuffizienz 2410*

Atemluft und Co-Vergiftung 1692

Atemwegserkrankungen des Bergmannes 2375

ATP und nephrogene Enzephalopathie $\mathbf{1 7 4 6}$

${ }^{198} \mathrm{Au}$-Kolloid bei Leberszintigraphie 1574

Audiometrie, Leitfaden 2252*

Aufklärung und Leberbiopsie 1463

Auge und Goldtherapie 2389

- und Ovulationshemmer 1761

Augenbank in Würzburg 1588

Augenheilkunde, Grundriß $1898^{*}$
Augenmuskellähmungen und Enteroviren 2073

Augenmuskeln und Zoster ophthalmicus 1865

Augennetzhaut und Dihydralazin 1688

Aureotan zu Erythematodes 2040

Ausbildung, ärztliche 1773*

Ausbreitungsfähigkeit der Blutplättchen 1735

Auskunftspflicht des Privatversicherers $2242 \mathrm{R}$

Auslandsreisen für Fortbildung $1704 \mathrm{R}$

Aussatz in New York 2130

Austauschtransfusion und Leberkoma 2235

Australia-Antigen 2294

Autoantikörper-Anämie und $C^{\prime} 3$-Gehalt 1980

Azathioprin, immunsuppressive Therapie $\mathbf{2 4 4 4}$

- und Knochenmark 2268, 2568

\section{B}

$\mathrm{B}_{12 \mathrm{2}}$-Avitaminosen, Funktionspsychosen 1473, 1945

B-Virus und Impfstoffe 166

Baader-Preis 19691509

Bactrim bei Bronchitis 2235

Baden und Trichomonaden 2524

Bakteriologe, Erinnerungen 1628*

Bakteriurien in der Schwangerschaft 1553

Ballonkatheder und Arterienverschluß 2317

Banti-Syndrom und Thrombozytenfunktion 1738

Bananengenuß und Niereninsuffizienz 1765

Banocide bei Filariose $\mathbf{1 4 5 7}$

Bartter-Syndrom 1445

Basensequenzanalyse von VirusRNS 2254

Bauchdeckenerschlaffung und Magenblutung 2241

Bauchlage beim Säugling 2450

Bauchspeicheldrüse, Reizung und Serumanalyse $\mathbf{2 0 8 6}$

Bechterew, Röntgentherapie 1505

- Thorium X 2459

Bechterewsche Krankheit 1586"

Befunde, ärztliche und Weitergabe $1503 \mathrm{R}$

Begutachtung durch Medizinalassistenten $2243 \mathrm{R}$

Beinamputation und Wirbelsäule 1706

Belastungselektrokardiogramm 2182

Belegarzt, Rechtsfragen 2616 R

Benzofuranderivate und Harnsäureausscheidung 2652

Benzol-Leukämie 1665, 1703

Bereitschaftsdienst in Kliniken $2298 \mathrm{R}$

Berger, H. und Elektroenzephalogramm 2123

Bergmann, Atemwegserkrankungen 2375

Bertram Preis 1588

Berylliose 2290

Bettruhe bei Hepatitis 2362

- und Mumpsmeningitis 2004
Bevölkerung und Krebsvorstellungen 1582

Bevölkerungszunahme 1899

Biber-Preis 1469

Biglieri-Syndrom 1445

Bilirubinkonzentration im Fruchtwasser $\mathbf{2 6 0 2}$

Bilivistan zur InfusionsCholezytographie 2018

Bindegewebsstoffwechsel und Vitamin D 1877

Biochemie 2361*

- der Porphysia cutanea tarda 1578

Biokybernetik 1953*

Biomedizin und Telemetrie 2649

-, Zusammenschluß von Firmen 1588

Blase und Reflux 2054

Blasenstörungen, neurogene $2460^{*}$

Blendenschutzbrille, gelbe 1545

Bleomycin 2249

Block, atrioventrikulärer bei Achalasie 2191

Blue-Rubber-Bleb-NaevusSyndrom 1991

Blut und Azathioprin bei M. Still 2268

- und Benzol 1665

- und Chloramphenicolschädigung 2241

-, Sauerstofftransport 1707"

- und Strahlentherapie, Echinacin-Test 1410

Blutalkohol, Rückrechnung 2259

Blutbild und CyclophosphamidStöße 2585

Blutdruck und CSN-Stimulator 1719

- nach Nephrektomie 2083

- und Nephrose 1674

- und Octapressin 1679

- und Ovulationshemmer 1764

- und Phäochromozytom 2299

- und Plasma-Renin 2267

-, Untersuchung 2358

Blutdruckmessung und Armumfang 1950

Blutgefäßsystem und Polyarthritis 2644

Blutgerinnung bei Moschcowitzsyndrom 2329

Blutgruppe und Leberzirrhose 1828, 2655

Blutgruppensystem $\mathrm{Xg}^{\mathrm{a}}$ und Geschlechter 2066

Bluthochdruck 2704

Blutkrankheiten, LDHAktivitäten 1882

Blutplättchen, Funktionsprüfung 1735

Blutsenkung und Leberkrankheiten 2007

Blutspende durch Polyzythämiker 2250

Blutspurendiagnostik 1548*

Blutung, ösophageale und

Therapie $\mathbf{1 4 7 7}$

-, subarachnoidale und Hydrozephalus 1617

- bei Hämophilie 1886, 2540

Blutverlust und Skelettfehlbildungen 2342

Blutvolumen, Substitution nach Herzschock $\mathbf{1 7 5 4}$

Blutzucker und Leberzellkarzinom 1781

Bonewand-Preis 1588

Borsäure bei Säuglingen 1841

Botulismus, Seuchenhygiene 1666

Bradykinesie und L-Dopa $\mathbf{2 6 8 0}$

Braunülen für Infusion 1794

Bronchialkarzinom 2180

-, Behandlung 1559

- und Cyclophosphamid 2588

- und LDH 1883

-, Nachbestrahlung 1598

-, Strahlenbehandlung 1593

-, Vorsorgeuntersuchungen 2569

Bronchialsystem, Entwicklungsanomalien 2185*

Bronchialwiderstand nach Pilokarpin 1651

Bronchialwiderstand, Werte 1785

Bronchien, Bildband 2064*

Bronchitis $1467^{*}$

-, asthmatoide und Differenzierung 1803

- bei Bergleuten 2375

-, chronische $2128^{*}$

,-- und Therapie 2234

Brown Boveri und HoffmannLa Roche, Zusammenarbeit 1588

Brustwandtumor, Diagnose 1725

Buchillustration, medizinische 2253*

Bürger-Preis 19692254

Bundesgesundheitsrat, Mitglieder 1509

Bundesprothesenliste 2361*

Bundesstatistik über Geschlechtskrankheiten 2254

Busulfan, immunsuppressive Therapie 2444

Butidrine 2560

Butyrylcholinesterase, Sequenz 2696

\section{C}

$C^{\prime} ;$-Komplementanalysen 1978

Calcium und Digitalis 2062

- und Vitamin D 1997

Calciumspiegel und Vitamin D 1877

Calciumstoffwechsel und Knochen 1774*

Calciurie nach PAS-Calcium 1928 
Chagaskrankheit und Herz-

beteiligung $\mathbf{1 7 5 8}$

Chefärzte, beamtete und

Anweisungsrecht 1544

Chemie, klinische $1844^{*}$

-, organische $1587^{*}$

-, physiologische $1586^{*}$

Chemoprophylaxe der Grippe 2511

Chemotherapie, Fortschritte 1774 :

-, Kongreß 2249

Chinidin und Antikoagulantien 1845

Chirurgie, Fortschritte 1468*

-, Geschichte allgemeiner 2662*

- bei Hämophilie A 2539

-, Komplikationen $2128^{*}$

- der Koronarien 1903

-, Lehrbuch 1547:

- und Organverpflanzungen 2082

-, Pathologie 1708*

-, spezielle für Praxis 2064:

- der Subaortenstenose 2579

Chlor im Schweiß und Mucoviscidose $\mathbf{1 6 7 7}$

Chlorambucil, immunsuppressive Therapie $\mathbf{2 4 4 4}$

Chloramphenicol und Bronchitis 2234

-, Knochenmarksschädigung 2240

- und Nocardiose 2507

- und Pyelitis 1939

- und Pyelitis beim Kind 2356

- und Schwangerschaftsbakteriurie $\mathbf{1 5 5 5}$

Chloride und Diuretika 1573

Chlormercuribenzoat und Tollwutvirus $\mathbf{1 7 0 1}$

Chlormethiazol und Suchtgefahr 2299

Chlorphenthixen, Wirkung 1943

Chlorprothixen 1943

Cholangiographie, direkte 2111

- und Hyperthyreosen 2632

Cholecalciferol, Wirkungsmechanismus 1996

Cholera-Schutzimpfung 1425

Cholesteatomeiterung, Schichtuntersuchung $2704:$

Cholesterin und Alter 1546

- und Diättherapie 2309

Cholezysto-Cholangiographie 2019

- -, direkte 2111

Chondroitinschwefelsäure und Perichondritis 2039

Chorea Huntington, PlasmaAminosäurespiegel 2411

Chorea major 2703

Choriomeningitis-Virus und Impfstoffe 1663

Chromosomenanomalien be Anstaltspatienten $\mathbf{2 6 7 5}$

Chromosomenstörungen 2622*

Chryseosis 2389

Chymotrypsin in Arzneien 1870

-, Sequenz 2696

- und Tetrachlorkohlenstoffvergiftung 1650

Ciatyl, Wirkung 1943

Circuit-Training 2126

Clearances, renaler 2685

Cloxacillin 2608

Clumping-Faktor der Staphylokokken 2352

${ }^{57} \mathrm{Co}-\mathrm{Cyanocobalamin}$ und Glomerulumfiltrat $\mathbf{2 6 8 5}$

Co-Vergiftung, Diagnostik 1692
Coca Cola und Glaukom 2529

Coeliakic Jugendlicher und

Katamnesen 2206

-, Katamnesen 1856

Coeliacographie $\mathbf{2 3 9 5}$

- und Röntgenverbesserungen 1485

- mit Sekretin und Adrenalin 1971

Coenzym und Enzyme 2693

Coeruloplasmin, erhöhtes im Konservenplasma 2706

Coffein bei Glaukom 2529

Coffeinprobetrunk, Wert 1666

Cohn-Fraktion bei Hämophilie A 1887, 2541

Colistin bei Pyelitis 1939

Colitis ulcerosa und $\mathrm{C}_{3}^{\prime}$-Gehalt 1980

- und Immunsuppression 2447

Colonresektion bei Hämophilie A 1799

Coma diabeticum, Testfehler 1566

- hepaticum 1829, 2238

Combizym, Aktivität 1871

Conn-Syndrom, Reninaktivität 1417

Corticoide, Hemmwirkung verschiedener $\mathbf{2 4 8 8}$

- und Hepatitis 2700

- und Immunsuppressiva $\mathbf{2 4 4 6}$

Corticosteroide oder ACTH? 1771

- bei Bronchitis 2234

- bei Perichondritis 2039

- und Transplantationslunge 2204

Cortison und Heparinbehandlung 2337

- und Zahngranulom 1467

Court-Bonewand-Preis 1588

Coxsackic und ECHO-Viren, Phänokopic 1965

Coxsackieviren und Nephropathie 2302

- und Nervensystem 2072

${ }^{51} \mathrm{Cr}$-EDTA-Clearance und Dihydralazin $\mathbf{1 6 8 8}$

CSN-Stimulator bei Angina pectoris 1717

Cumarinderivate und Harnsäureausscheidung $\mathbf{2 6 5 2}$

Curt-Adam-Preis 19702011

Cyclamat-Bann in USA 2462

-, karzinogene Wirkung? 2619

Cyclophosphamid bei Erythroleukämie 2420

-, immunsuppressive Therapie 2444

- bei Leukämie 1993

-, ultrahohe 2583

Cystic-Fibrosis-Analyzer 1677

Cytarabin bei Leukämie $\mathbf{1 9 9 3}$

Cytosin-Arabinosid und

Erythroleukämie $\mathbf{2 4 2 0}$

Czerny-Preis 2362

D

Daonil, Proteinurie 2703

Darm- und Cyclophosphamid 2591

- und Xyloseresorption bei Alkoholismus 1536

Darmflora und Lysozym 1461

Darmresektion, Fettemulsionen und Ikterus $\mathbf{1 8 2 5}$
Daunorubicin bei Leukämie 1993

- bei Leukämie des Kindes 1972

Debilität und Chromosomenanomalien $\mathbf{2 6 7 6}$

Decarboxylasehemmer M. Parkinson-Therapie $\mathbf{2 6 7 8}$

Decortin bei Hepatitis 2700

Defektsyndrome bei $\mathrm{B}_{12}$ Avitaminosen 1475

Deferoxamin und Urineisen bei Kindern 1410

Demenz, senile 2185

Depostat und Endometriumkarzinome 2472

Dermatitis, rosacea-artige 1393

- seborrhoides des Kindes 1841

Dermatologie, A-Z 2461*

- Atlas 1508*

Dermatomyositis und C':3-Gehalt 1980

Desinfektion $1427^{*}$

- nach Mumps-Meningitis 1585

Desmoidtest, Wert 1666

Desoxyribonucleinsäure, Aufklärung 2410*

-, Entdeckung 1948

- und Phytohämagglutinin 1868

Dextran-Isoprenalin bei InfarktSchock 1643

Diabetes und Cyclamat 2619

-, Enteropathie 1950

-, Förderpreis 2067

- und Foetopathia pseudodiabetica 2010

- und Gallenblasengröße 2186

- nach Gonadotropinen? 1626

-, Hafertage 1771

- und Hyperlipidämien 2311

-, idiohypophysärer 2028

- und Kind, Nahrungsbedarf 2535

-, Pathophysiologie 2301*

- und Teststreifen-Fchluntersuchungen 1566

- und Zuckeraustauschstoffe 2659

Diät bei der Einkommenssteuer $2243 \mathrm{R}$

- und Hepatitis 2362*

Diätetik bei Kranken 2360*

- der Niereninsuffizienz 2113

- der Nierenkrankheiten 2252"

Diäthylcarbamazin bei Filariose 1457

Diättherapie der Hyperlipämie 2307

Diagnostik 1668

-, aktuelle $2008^{\circ}$

-, chirurgische 1952*

-, Klinik 2659

-, Manual 1468

Dialyse und Flüssigkeits-Bilanz 2059

- bei Nierenkrankeri $\mathbf{1 8 8 8}$

- nach Tetrachlorkohlenstoffvergiftung 1650

Diarrhoe, chologene 1545

Diathese, hämorrhagische und Thrombozyten 1953:

Diazepam, Nebenwirkungen 2571

Dibutil, Wirkung 1943

Dickdarm, Harnableitung 2116

Dickdarmkrebs, Frühdiagnose 2186

Dicloxacillin 2608

Diethazin, Wirkung 1943

Digizalis und Calcium 2062

Digitalisresorption bei Rechtsherzinsuffizienz 2359

Digitalistherapie und

Hirndurchblutung 2182

Di-Guglielmo-Syndrom des

Erwachsenen 2421

- - im Kindesalter 2415

Dihydralazin und Nierendurchblutung 1688

Diparcol, Wirkung 1943

Dipyridamol und Herz 2564

Distraneurin, Suchtgefahr 2299

Distreß bei Neugeborenen 1563

Diurese, forcierte 1896

- und Oxytocin 2612

Diuretika, Wurzelreizerscheinungen? 2408

Diuretikaabusus, Kaliumverlustsyndrom 2634

Diuretikatherapic bei Niereninsuffizien 1558

Divertikulosis des Dünndarm 2097

DNS, Entdeckung 1948

- Viren und Zelle 1629

Dociton 2560

Dokumentation, Deutsches Institut 1899, 2706

Dopamin und Parkinson 1942

Doppel-Helix 2410*

Down-Syndrom u. Chromosomen 2676

Druckfehler, medizinische und Haftung $1839 \mathrm{R}$

Dünndarm bei Coeliakie 1861, 2210

Duinndarm, Harnableitung 2117

Dünndarmventrikel, Klinik 2097

Duodenalgeschwüre und Leberzirrhese 2167

Duodenalkompression, arteriomesenteriale $\mathbf{1 4 9 5}$

Duodenalsaft, Porphyrine 2392

Duodenalulkus und Alkalose 1446

Durchblutungsstörungen, Therapie peripherer 2347

Dysgammaglobulinämie, C':3-Gehalt 1980

Dysrhythmien im EEG $2572 *$

E

Echinacintest, Knochenmarkreserve bei Strahlentherapic 
Ekzem, seborrhoisches und rosaceaartige Dermatitis 1395

Elastase, Sequenz 2696

Elektrochirurgie und Schrittmacher-Störung 1469

Elektroenzephalogramm, Dysrhythmien 2572

- und H. Berger 2123

- und Hirntod 2119

- und Mumpsmeningitis 1599

Elektrokardiogramm und Aortenstenose 1538

- und Herzrhythmusstörungen 2671

-, Hypokaliämie 1841

- und Lungeninfarkt 2607

- und Myokardiopathie 1756

- der Subaortenstenosenoperation 2581

Elektrolyte und nephrogene Encephalopathie $\mathbf{1 7 4 3}$

Elektrolytersatz und Blutverlust, Skelettfehlbildungen $\mathbf{2 3 4 2}$

Elektrolythaushalt, postoperativer 1898:

Elektromagnetik der Stromzeitvolumina 1621

Elektronenmikroskopie, Raster1832

Elektronik und Röntgenaufnahmen-Verbesserung 1483

Elektrophorese beim MarfanSyndrom 2284

Embolie, arterielle 2315

- am Auge und Ovulationshemmer 1764

-, Fett- 2690

- und Thrombolyse 2292

Empfängnisverhütung 1952"

Endarterektomie am Herzen 1904

Endokard-Fibroelastose und Mumps 2529

Endokrinium und Leber $1627^{*}$

Endokrinologie, gynäkologische 23.00*, 2621*

Endokrinopathien und Kaliumverlust $\mathbf{2 6 3 5}$

Endometriumkarzinome Schutzmechanismus 2467

Endomyokardfibrose $\mathbf{1 7 5 5}$

Endopeptidasen und Aminosäuresequenz 2696

Endoplastitiden 1448

Endoradiosonde als $\mathrm{pH}$-Indikator 2274

- und Telemetrie 2651

Endoskopie der Magenkarzinome $\mathbf{2 2 4 0}$

- der Papilla Vateri 2575

Endoxan bei Amyloid 2692

- bei Leukämie 1993

Enteritis und Lysozym 1460

Enterokokken, Metastasen der Lunge 2227

Enteropathie, diabetische 1950

Enteroviren und Nervensystem 2071

Enzephalitiden bei Herpangina 1964

Enzephalitis und Amöben 2186

- und Enteroviren 2071

-, luische und Therapie 1489

- bei Mumps 1549

-, perivenöse und Meningitis 1532

Enzephalopathie und Kalium/ Calzium-Quotient $\mathbf{1 7 4 3}$

Enzymaktivitäten und Lebererkrankungen 1914
Enzymase, Aktivität 1871

Enzymdefekt, neuer 2002

Enzyme, abnorme und Gargoylismus 1469

-, lysosomale bei Glykogenose Typ II 1415

-, Röntgenkristallographie 2698

-, Struktur und Funktion 2705"

-, Zentrum 2693

Enzymgehalt von Pankreasfermentpräparaten $\mathbf{1 8 7 0}$

Enzymmangel bei Pompescher Krankheit $\mathbf{1 4 1 5}$

Enzymologie der Arterienwand $2129 *$

Enzymtherapie der Tumoren 1669

Epidermolyse, toxische 1422

Epilepsie und Chromosomen 2676

Epilepsien 2526"

Epistaxis bei Blutern 1886

Epithelkörperchenadenome und Hyperparathyreoidismus 2593

- nach Nierentransplantation 2143

Erkältung und Infektbahnung 1771

Ernährung bei Diabetes des Kindes 2536

- und Herz 1759

Erwachsene und Komplementanalysen 1979

Erythematodes und C':3-Gehalt 1980

Erythematodes durch Goldtherapie $\mathbf{2 0 4 0}$

Erythrämie 2419

Erythroblastose und Fruchtwasser-Bilirubin 2603

- des Erwachsenen 2421

-, Prophylaxe 1654

Erythroleukämie 2415

Erythrozyten, fetale und Gravidität 1435

- und Imurel 2269

- und Thrombozyten 2360*

Escape-Phänomen 1442

Escherichia coli und Carbenizillin 2501

Esterasen, Aminosäuresequenz 2696

- und Toxogonin 1583

Ethacrynsäure bei renaler Acidose 1398

- und Niereninsuffizienz 1568

Euglucon, Proteinurie 2703

Europa, Straßenverkehrsunfälle 2529

Euthyreose, Thyroxin und Jod 2386

Evokationstest des Pankreas 2086

Extrasystolie, Therapie $\mathbf{2 6 7 0}$

Extremitätenamputation, Leukämie 2571

\section{F}

Fachkongreß und Persönlichkeitsbildung $1704 \mathrm{R}$

Facialislähmung und Enteroviren 2073

Faktor VII und Wunddehiszenzen 1405

Faktor VIII und AHG 1887

- und Hämophilie 1885

- und Kryopräzipitate 1799
Farbfotographie in der Medizin 1549

Farbsehstörungen und Leberzirrhose $\mathbf{1 8 2 8}$

Farmerlunge und Hühnerzüchterlunge 2627

Faszienklappenplastik, Hämolyse 2212

Felypressin-Infusion und Pfortaderdruck 1679

Fernsehmikroskopie $\mathbf{1 8 3 4}$

Fernsehschäden bei Kindern 1453

Festal, Aktivität 1871

Fette und Hyperlipidämien 2307

Fettembolie, Diagnose 2690

Fettemulsionen und Leberentzündung $\mathbf{1 8 2 5}$

Fettleber und Alkohol 1543

- und Eisenstoffwechsel 1841

- und $\gamma$-GT-Aktivität 1911

-, Therapie 1619

Fettsäuren, veresterte und Diät 2309

Fettzirrhose und Magenresektion 2169

Fetus, Geschlechtsdiagnose 2663

- und Komplementfaktor C': 1979

Fibrinogen und Wunddehiszenzen 1405

Fibrinolyse $\mathbf{2 2 9 2}$

- und Herzschock 1754

Fibroelastose 1757

- und Mumps 2529

Fibrose, cystische und Diagnose 1677

Fibrosen, Einteilung 2290

Fieber, hämorrhagisches in Weißrußland 2462

- durch Nitrofurantoin 1922, 2523

rheumatisches 2007

Fieberkrämpfe und Pockenimpfung 2403

Fiebertherapie der Paralyse 1486

Filariose, Therapie $\mathbf{1 4 5 7}$

Filtrationsrate, glomeruläre 2685

Fistel und Hämodialyse 1604

-, transpulmonale, arteriovenöse 1917

Flüssigkeit beim Hitzschlag 1696

Flüssigkeitsbilanz bei Peritonealdialyse 1873, 2059

Fluocortolon und Hemm wirkung 2491

Fluoreszenzangiographie, Atlas $1587^{*}$

Fluoreszenz-Intravital-

Mikroskopic $\mathbf{1 8 3 3}$

Fluoridtherapie der Osteoporose 1730

Fluorphenylalanin bei Virus-

krankheiten 1541

5-Fluoruracil, immunsuppressive Therapic $\mathbf{2 4 4 4}$

Flury HEP Stamm des Tollwutvirus $\mathbf{1 7 0 0}$

Flußlymphographie, Kontrastmittelspeicherung der Leber 2547

Flußmessung, elektromagnetische 1621

Foetopathia pseudo-diabetica 2010

Forbessche Krankheit $\mathbf{1 4 2 0}$

Fortbildung des Arztes $1773^{*}$

-, Erleichterung 1543

Fortbildungszweck von Auslandsreisen $1704 \mathrm{R}$

Fraktion I nach Cohn 2541

Framingham-Studie 1667

Frauenmilch und Lysozym 1458

Fremdviren in Impfstoffen 1657

Freon und Tollwutvirus 1701

Frerichs-Preis 1970 2131

Frey-Preis 19702067

Fruchtwasser, Bilirubin-

konzentration 2602

Frühdiagnosenstellung $1844^{*}$

Frühgeburt, Vibrio-fetus-Infektion 2487

Frühkarzinom des Magens 2441

Fundusphotographie und Nepresolwirkung 1688

Fungizone bei Lungenmykosen 1992

Funktion und Struktur 2129:

Funktionspsychose bei $\mathrm{B}_{12}$ Avitaminose 1473, 1945

Furacin und Allergie 1686

Furosemid und Niereninsuffizienz 1568

Fusidinsäure und Nocardiose 2506

Fußbeschwerden und Beinamputation 1706

\section{G}

Gänssler, M. † 1583

Gallenblase, Erkrankungen und Cholezystographie 2111

Gallenblasengröße und Diabetes 2186

Gallengangsnekrose nach Lebertransplantation $\mathbf{1 7 1 3}$

Gallenwege, Chirurgie 2064*

-, Erkrankungen 2459*

-, Kontrast-Infusion 2018

-, Röntgenkontrastmittel und Hyperthyreose 2631

Gallenwegserkrankungen und $\gamma$-GT-Aktivität 1913

Gallenwegserkrankungen und LDH 1884

Ganzkörperplethysmographie und Lungenfunktion $\mathbf{1 7 8 5}$

Ganzwortmcthode und Legasthenie 1462, 1893

Gargoylismus und Enzyme 1469 
Gefäßkrankheiten 2704"

-, Taschenbuch $1627^{*}$

- und Thrombozyten 1953"

Gefäßverschliisse am Auge und

Ovulationshemmer $\mathbf{1 7 6 2}$

Geflügelviren in Impfstoffen 1659

Gehirn und Meningitis purulenta

1529

- und Parkinsonismus 1943

-, Tuberkulose 2281

Gehör, Operationen zur

Verbesserung $1773^{*}$

Gelenkblutungen bei Hämophilie $\mathbf{1 8 8 6}$

Gelenkschäden nach Beinamputation 1707

Genetik, humane 2410"

-, Schliissel 2066

- Wörterbuch 2129:

Gentamycin bei Nocardiose 2506

Gentianaviolett bei Mykosen 1993

Gerinnung bei Hämophilie B, 2145

-. und Moschcowitz-Syndrom 2330

Gerinnungsfaktoren und Wunddehiszenzen 1401

Gerinnungshemmung und

Tumoren 2706

Gerinnungsstörungen bei Hitzschlag 1696

Germaniumelektroden 2706

Germinoblastom und Lymph knoten 2199

Geruchshalluzinationen 2618

Geschlechter und $\mathrm{Xg}^{\mathrm{a}}$-Blutgruppensystem 2066

Geschlechtsdiagnose vor der Geburt 2663

Geschlechtskrankheiten, Bundesstatistik 2254

Geschwisterzahl und Schulcrfolg 1629

Gesellschaft für Immunologie 1777

- für Phlebologie und Proktologie 2187

Gesicht, Humangenetik 1951"

-, Operationen 1586"

Gesichtsentwicklung 1898"

Gestagene und Endometriumkarzinom 2472

- zur Karzinomthetapie 2408

Gesundheit und Rauchen 1509

Gesundheitsamt und Krankheitsmeldung $1767 \mathrm{R}$

Gesundheitswesen, Ausgaben 2575

Gewebe und LDH 1882

- Sauerstofftransport $1707^{\circ}$

Gewebsverkalkungen und Vitamin D $\mathbf{1 8 7 7}$

Gewöhnung, Grundlagen 2065

Gicht- und Indandionderivate 2653

-, Röntgenbefunde 1909

van Gierke Glykogenose 1420

Glaukom, Asthmatiker und Pilokarpin 1651

- und Coffein und Coca Cola 2529

Glibenclamid, Proteinurie 2703

Gliedmaßenarterien, Arterienverschluß 2318

Gliedmaßenmangel und Phantome 2367
Glomerulonephritis und Acidose 1399

- und C':3-Gehalt 1980

-, chronische und Hämodialyse 1605

- bei Good-pasture-Syndrom 1820

- und Nephrose 1673

Glomerulumfiltrat, Bestimmung 2685

- und Dihydralazin 1688

Glucagon bei Hypoglykämie Tumorkranker $\mathbf{1 7 8 1}$

- und Wachstumshormon 1509

Glucokortikoide oder ACTH 1771

- in der inneren Medizin 1427*

- und Proteinsynthese der Leber 1615

Glucose, Assimilationskoeffizient 2458

- Urinuntersuchung und Fehlbeurteilung 1566

Glucosebelastungstest 1706

$\alpha-1,4$-Glucosidase-Mangel bei Glykogenose 1414

$\beta$-Glucuronidase und Pompesche Krankheit $\mathbf{1 4 1 4}$

$\gamma$-Glutamyl-Transpeptidase, Aktivität bei Lebererkrankungen 1911

Glycyrrhicinsäure und Magenulkus 2566

Glykogenose, muskuläre $\mathbf{1 4 2 1}$

- Typ II 1414

Glykogenosen, Biochemie und Klinik 1420

Glykoside bei Herzschock 1753

Glykoprotein und Pneumokokkenmeningitis $\mathbf{2 1 5 0}$

Goldtherapie und Augenbeteiligung 2389

- zu Erythematodes 2040

Goodpasture-Syndrom $\mathbf{1 8 2 0}$

- - und Alport-Syndrom 2637

Gordhkanüle bei Infusionen 1794

GOT und Lebertransaminasen 2250

GPT und Leberleiden 2250

Granulozyten und Azathioprin 2269

Granulozytopenie 2182

Gravidität und Anti- $\mathrm{Rh}_{0}$ (D)

Prophylaxe 1433

- und Röntgenreihenuntersuchung 2127

Grenzdextrinose nach Forbes 1420

Grippe und Sterbefälle 1777

Grippeprophylaxe, Möglichkeiten 2510

Großstadt und Unfälle 2066

Gutachten, Medizinische 2300*

Gutachtertätigkeit und Umsatz steuer 2454 R

Gynäkologie, Endokrinologie $2300^{*}, 2621^{*}$

- und Geburtshilfe 162 *

\section{$\mathrm{H}$}

Haar, Psoriasis 1466

Hämagglutination und Tollwutvirus 1698

Hämangiome 1990

Hämangioperizytom 1506

Hämatologie, Diagnostik 1426

-, praktische $2661^{*}$
Hämatome bei Hämophilie 1886

Hämaturie bei Blutern 1886

Hämodialyse und QuintonShunt 1604

- bei Malaria 1520

- und Niereninsuffizienz 2262

Hämoglobinresorption, enterale 1950

Hämoglobinurie, paroxysmale, näch tliche 1891

Hämolyse, traumatische bei Faszienklappenplastik 2212

$\alpha$-Hämolysin und Staphylokokken 2349

Hämopexin bei Pneumokokkenmeningitis $\mathbf{2 1 5 0}$

Hämophilie, Therapie $\mathbf{1 8 8 5}$

- A und chirurgische Eingriffe 2539

- A, Colonresektion 1799

- B, Tumoroperation 2145

Hämosiderose der Lunge Immunsuppression $\mathbf{1 8 1 3}$

Hämostase und Thrombozyten 2527 :

Hafertage bei Diabetes 1771

Hals-Nasen-Ohren-Heilkunde $2183^{*}$

Hals-Nasen-Ohren-Krankheiten, Bildband 2064*

Hals, Operationen 1586*

Halswirbelsäule, Syndrome $2527^{*}$

Hamperl, H. 70. Geburtstag 1894

Hansen-Gedächtnispreis 2463

Haptoglobin und Pneumokokkenmeningitis $\mathbf{2 1 5 0}$

Harnableitung, künstliche 2115

Harnblasenersatz 2117

Harnelektrolyte und Osteopathie 2336

Harnleiter und Reflux 2054

Harnröhrensteine, Therapie 2384

Harnsäureausscheidung und Cumarin-Indandion und Benzofuran-Derivate 2652

Harnsäurespiegel und Gicht 1910

Harnsäuresteine, Therapie 2384

Harnstein, akuter 2381

Harnsteine nach PAS-Calcium 1929

Harnuntersuchung, chemische und mikroskopische $1775^{*}$

Harnwegsinfektionen und Penicilline $\mathbf{2 6 1 0}$

Harnwegsinfektionen in der Schwangerschaft 1555

Hartmetall-Lungen 2290

Haut und Porphyria cutanea tarda 1578

Hautdurchblutung, Steigerung 2348

Heilbehandlung, physikalische und Beschreibung 1469

Heilmeyer, L. in memoriam 2657

-, Gedenkmünze in Gold 2707

Heimdialyse, Probleme $\mathbf{1 8 8 8}$

Helopanzym, Aktivitäten $\mathbf{1 8 7 1}$

Hemmkörperhämophilie, Therapie $\mathbf{1 8 8 7}$

HEP-Flury-Stamm des Tollwutvirus 1699

Heparin und Herzschock 1754

- bei Moschcowitz-Syndrom 2327

Heparinbehandlung, Osteopathie 2334

Hepatitis und Australia-Antigen 2294

und Bettruhe und Diät 2362

- und Blutgruppe 2655

- und C':3-Gehalt 1980

-, chronische und Laparoskopie 2121

-, - und Prognose 2215, 2220

-, - und Therapie 2397, 2700

- und $\gamma$-GT-Aktivität 1911

- bei Herpangina 1964

- und Immunsuppressiva 2447

- und Laparoskopie 1633

- und LDH 1884

-, lupoide und C':3-Gchalt 1980

- und Narbenleber 1637

- und Proteinsynthese nach Glucocorticoiden 1616

- und Resochin 2299

Hepatographie, Kontrastmittelspeicherung 2547

Hepatom, malignes und Lebertransplantation 1713

Hepatose, cholestatische und $\gamma$-GT-Aktivität $\mathbf{1 9 1 4}$

Hepatosplenomegalie und Zytomegalie 1969

Herdnephritis und Nephrose 1674

Herpangina, Epidemie 1959

Herpes-Viren 2662*

- und Impfstoffe 1659, 1660

Herter-Heubner-Krankheit, Katamnesen 1856

Herz und Digitalis, Calciumgabe 2062

- Glykogenose 1421

- und Hitzschlag 1696

- kardiogener Schock 1752

- und Neugeborenen-Mortalität 1564

-, Pathologie 2622.

- und Polyarthritis chronica 2644

- und Polychondritis 2039

- und Revascularisation des Myokard 1903

-, Tachykardie im Anfall 2574*

- und Vitamin D 1879

Herzamyloid, seniles $\mathbf{2 1 7 0}$

Herzchirurgie und elektromagnetische Flußmessung 1622 
Herzrhythmusstörungen und d-Propranolol 2669

Herzschlagvolumen und

Quinton-Shunt 1604

Herzschrittmacher und Elektrochirurgie 1469

Herzstillstand bei Achalasie 2191

Herzsyndrom, hyperkinetisches

Herztransplantation des Höllensekretärs Lu 1945

Hetacillin 2608

Hetrazan bei Filariose $\mathbf{1 4 5 7}$

$\mathrm{Hg} \mathrm{Cl}$ und Tollwutvirus 1702

Hilfspersonal, Haftung bei Fehlern $1946 \mathrm{R}, 2240,2656 \mathrm{R}$

Hirnchirurgie und Mikrochirurgie 1496

Hirndurchblutung und Digitalistherapie 2182

Hirnnervenlähmung durch Enteroviren 2071

Hirnrhythmen, Hans Berger 2125

Hirntod 2183*

-, dissoziierter 2118

-, Feststellung 2404

Hirntrauma und Hydrozephalus 1617

Hirntuberkulome, Differentialdiagnose 2281

Hirntumoren, Liquordrainage 1418

Hirnvenenthrombosen nach Meningitis 1531

Hirnveränderungen und Mangelernährung intrauterin 2130

Histochemie $2253^{*}$

Histologie und Zytologie 2300*

Hitzeberuf und Nierensteinleiden 1666

Hitzschlag, Therapie 1695

HNO-Spiegelkurs 1897"

Hochschulen, Ausgaben 2130

Hochvolttherapie des Bronchialkarzinom 1596

Hodgkin, Lymphknotenbeteiligung 2199

Hodgkin-Sarkom und Cyclophosphamid 2588

Hodenphantome 2368

Hoffmann-Medaille 1470

Homburg-Preis 2011

Honig und Pollenallergie 2458

Hora incerta 1845

Hormone bei gynäkologischen Karzinomen 2408

-, neuro-hypophysäre $1773^{*}$

Hornhaut und Goldtherapie 2391

Hornhautgewebe, Augenbank 1588

Horoskop des Dr. med.

L. Rauwolff $\mathbf{2 2 4 5}$

Hospitalismus $\mathbf{1 4 4 8}$

Hühnerviren und Impfstoffe 1659

Hühnerzüchterlunge 2627

Hufeland-Preis 1588

Humangenetik $1951^{*}, 2301^{*}$, 2410

Hunde, Tollwutschutzimpfung 2620

Hunter-Hurler-Syndrom 1469

Hyaluronidasemangel bei Glykogenose Typ II $\mathbf{1 4 1 4}$

Hyaluronsäure, Abbau 1416

Hydantoine und Erythematodes 2041

Hydergin und Hirndurchblutung 2429
Hydroxybenzylbenzimidazol bei Virusinfektionen 1542

Hydroxy-cholecalciferol 1955

- und Rachitis 1997

17 $\alpha$-Hydroxylase-Defekt 1442

Hydroxy-norprogesteroncapronat und Mitosenzahl im Endometriumkarzinom 2471

Hydrozephalus des Erwachsenen 1617

-, Liquordrainage 1418

Hyperaldosteronismus 1442

- und Kaliumverlust 2635

-, sekundärer 2049

-, Therapie 2051

Hypercalcämie bei Epithelkörperchenadenom 2595

- bei Myelom 2499

- nach Nierentransplantation 2143

- , toxische 2155

- und Vitamin D 1877

Hypercalciurie und Nephrolithiasis 1491

- unter PAS-Calcium 1928

Hypercorticismus, paraneoplastischer 1443

Hypergammaglobulinämien $1897^{*}$

Hyperinsulinismus 2458

Hyperkapnie und Alkalose 1441

Hyperlipämie, Diättherapie 2307

Hyperlipidämie und Framingham-Studie 1667

Hypernephrom und Cyclophosphamid 2588

Hyperparathyreoidismus nach Niereninsuffizienz 2140

- und Nierentransplantation 2084

-, primärer 2593

- tertiärer 2143

Hypertension, portale und Myelofibrose 2165

Hyperthermie und Hitzschlag 1695

Hyperthyreose, Jod und

Thyroxin 2386

- bei Jodzufuhr 2631

- und Trijodthyronin-Test 1456

Hypertonie 2704*

- und Alkalose 1441

- und Dihydralazin und Niere 1688

- und Hyperaldosteronismus 2050

-, maligne und Alkalose 1444

- und Phäochromozytom 2299

- und Rezeptorenblocker 2561

Hypnose, aktive $1708^{*}$

Hypocholesterinämie, enterogene 1545

Hypogammaglobulinämie, C'3-Gehalt 1980

Hypoglycaemia factitia 1502

Hypoglykämie, bei Leberzellkarzinom 1781

Hypokaliämie und metabolische Alkalose 1441

- im EKG 1841

Hypokinesie, Therapie 2678

Hypophyse und Diabetes mellitus 2028

- und Novothyral 2481

- und Ovulationshemmung 2476

Hypohyseninfarkt und Diabetes mellitus 2029

Hypophysentumoren, Behandlung 2442
Hypothyreosen und Novothyral 2479,2481

Hypotonie $\mathbf{2 1 7 2}$

Hypoxie und Herzmuskelnekrose 1852

I

Idoxuridin bei Viruskrankheit 1540

Ig A-Defekt und Malabsorptionssyndrom 2023

- - System und Bronchitispartien 2554

Ig G-anti-D bei Rh-positiven Personen 1440

- - System und spastische Bronchitis 2554

Ikterus und Enzymaktivitäten 1915

Ileumblase 2117

IMET 3393 bei Myelom 2495

Imidopazin bei Cyclophosphamid-Erbrechen 2584

Immundiffusionstechnik, radiale 1978

Immunelektrophorese bei Pneumokokken-Meningitis 2150

Immunglobulin-anti-D und Morbus haemolyticus neonatorum 1437

- - D und Rhesus-Sensibilisierung 1654, 1655

Immunglobuline, Bausteinanalyse 2623

- und Cyclophosphamid 2586

- und spastische Bronchitis beim Kind 2555

Immunglobulinmangel und Malabsorptionssyndrom 2023

Immunität der Transplantation $1708 \mathrm{R}$

Immunologie, Gesellschaftsgründung 1777

- und Lupus erythematodes, arzneimittel-induzierter 2341

- und Marfan-Syndrom 2289

- und Thymus 2063

Immunopathien, Langzeittherapie $\mathbf{2 4 4 4}$

Immunprophylaxe bei Grippe 2510

Immunreaktionen 2662 "

Immunsuppression, $C^{\prime}{ }_{3}$-Gehalt 1981

- mit Imurel 2272

- Komplikationen 2082

- und Lungenhämosiderose 1813

-, spezifische kombinierte 1421

Immunsuppressiva 2444

-, Schäden 2568

Immunthyreoiditis und Novothyral 2482

Immuntoleranz 1508*

Impfmalaria bei Neurolues 1486

Impfstoffe und Fremdviren 1657

Impfung bei Pocken 2403

- gegen Röteln 1626

Impfungen bei Blutern 1886

- und Erythematodes-

Auslösung 2041

Imurel und Hepatitis 2700

- und Knochenmark 2268

Indandionderivate und Harnsäureausscheidung 2652

Infarkt, intramuraler 1625

- und Schocktherapie 1642

-, Streptokinasetherapie 2296

Infekt und Erkältung 1771

Infektionskrankheiten, Erreger $2572 \%$

Infektionskrankheiten in den Ländern der Bundesrepublik Deutschland

20.4.-17.5. $1969 \quad 1428$

18. 5.-14.6.1969 1629

15. 6.-12.7. $1969 \quad 1776$

13.7.- 9.8. 19691954

18. 8. - 6.9. 19692463

Influenza und Penicilline 2609

Influenzaviren $2661^{*}$

Information, Dokumentation 2706

Infusionsbehandlung, intravenöse und Techniken 1793

Infusions-CholezystoCholangiographie 2018 Injektion 2361*

Innere Krankheiten, Differentialdiagnose $2660^{*}$

Innere Medizin, Lehrbuch 2128*

Insektizide im Krankenhaus 2126

Institut für Dokumentation 1899, 2706

Insuffizienz, zerebrale und Therapie 2429, 2654

Insulin und Hypoglykämie von Tumorkranken 1783

Intensivpflegezentrum, dissoziierter Hirntod $\mathbf{2 1 2 0}$

Interferonproblem 2130

Intoxikationen, Osmotherapie 1896

Intrakatheter-System $\mathbf{1 7 9 4}$

Inulin und Co-Cyanocobalamin Clearance 2687

Inulin-Hippuran-Nephrogramm und Dihydralazin 1688

Ismelin und Blutdruck 2083

$\mathrm{N}_{1}$-Isonicotinoyl-N2-3-methyl-4chlorbenzoylhydrazin bei Viruskrankheiten 1542

Isoprenalin und Asthma-Teste 1805

- und Myokardinfarkt und Schock 1640

Isotope, radioaktive und Mineralsalzbestimmung 1983

Isoxazolyl-Penicilline $\mathbf{2 6 0 8}$ 


\section{K}

Kälteschädigung der Zelle 1498 Kältesonde für Prostatavereisung 1499

Kälteurtikaria und Kininsystem 2054

Käsewascherkrankheit 1709

Kalium im Liquor und

Enzephalopathie $\mathbf{1 7 4 3}$

- und Niereninsuffizienz 2114

Kaliumbilanz bei renaler Acidose 1400

Kaliumexkretion und Diuretika 1572

Kaliumperjodat und Tollwutvirus 1702

Kaliumverlustsyndrom, renales 2634

Kallikrein-Kinin-System 1668*

Kalorienzufuhr bei Diabetes des Kindes 2535

Kampfer, Verwendung 1944

Kapazität der Lunge $\mathbf{1 7 8 5}$

Kardiologie, pädiatrische und Arbeitsgemeinschaft 2463

Kardiomegalie, Diagnostik und Therapie 1537, 1539

Karzinome und Cyclophosphamid 2583

- und Enzyınaktivitäten 1916

-, gynäkologische und Hormontherapie 2408

-, - und Schutzmechanismen 2467

- und Heparin 2706

- und LDH 1884

-, Lymphknotenmetastasen 2202

- und Polyneuropathie 2104

Karzinomdiagnose, angiographische des Oberbauches 2397

Kastration und Endometrium 2470

Katarakt durch Röntgenstrahlen 1585

Katheter der V. subclavia $\mathbf{2 6 8 2}$

Katheteruntersuchung des Herzens 2345

Keimbestimmung im Urin 2223

Keratitis herpetica, Therapie $\mathbf{1 5 4 0}$

Keuchhusten und Ampizillin 2435

Kind, Ampicillintherapie des Keuchhusten 2436

- und Aortenisthmusstenose 1522

-, Coeliakie und Katamnesen 2206

- und Dermatitis seborrhoides 184.1

- und Diabetes, Nahrungsbedarf 2535

- und Ganzwortmethode 1893

-, Di-Guglielmo-Syndrom 2415

-, Enteroviren und Nervensystem 2071

-, Fernsehschäden 1453

-, Fettemulsionen und Ikterus 1825

-, Gliedmaßenmangel und

Phantomschmerz 2372

-, Herpangina-Epidemie 1959

-, Ig A und Ig G bei Asthmabronchitis 2554

-, und Immunglobulin Anti-D.2 1438

-, Keimzahlbestimmung im Urin 2223
Kind, Klumpfuß-Risiko 1950

-, Leukämiebehandlung 1972 , 1993

- und Lungenhämosiderose 1813

-, Lysozym 1458

- und Mortalität, perinatal 1563

-, Neprologie 2621"

-, Opfer von Straftaten 1899

-, Paraleukoblastenleukämie 2550

-, Pneumokokkenmeningitis und Neuraminidase 2149

- und Pocken-Impfung 2403

-, Pyelonephritis-Therapie 1939

-, Pyelitis und Therapie 2356

-, und Reflux 2053

- und Schwangerschaftsbakteriurie 1556

-, Still-Syndrom und Azathioprin 2268

-, Thiamin-bedürftige Anämie 2000

-, Urineisenbestimmung 1410

- und Urolithiasis nach PASCalcium 1491, 1928

-, Vibrio fetus-Infektionen 2400

- und Vitamin D-Úberdosis 1878

- und Zytomegalie-Antikörper 1966

Kinderheilkunde für Schwestern $2573 *$

- Therapie 1507"

Kinderkliniken mit PilocarpinIontophorese $\mathbf{1 6 7 8}$

Kinderkrankenpflege für Schwestern $2573^{*}$

Kinderkrankenschwestern, Lehrbuch 2461"

Kindesalter, endokrine und genetische Störungen $2184^{*}$

Kinin-Kallikrein-System $1668^{*}$

Kininsystem und Kälteurtikaria 2059

Klimaanlagen in Arbeitsräumen 2251

Klimakammer bei Pertussis 1624

Klinefeltersyndrom bei Anstaltspatienten 2677

Klinik für Diagnostik 2659

Klumpfuß, Vererbbarkeitsrisiko 1950

Kniegelenk und Beinamputation 1706

Knochen und Calcium-Stoffwechsel 1774 *

-, kindlicher und Mineralsalze 1983

Knochenmark und Azathioprin 2268

- und Chloramphenicol 2240

- und Cyclophosphamid 2587

- und Daunoblastin 1974

- bei Echinacin-Test 1409

- und Polyarthritis chronica 2644

Knochenmarkschäden bei Immunsuppressionen 2568

Knochenstoffwechsel und Heparinbehandlung 2337

Knochensubstanzgehalt und Natriumfluorid-Therapie 1732

Kobalt und Herz 1759

Koebcke, H. 2701

Körperhöhe und Schulerfolg 1629

Körperkreislauf und Lungenkreislauf, Fistel 1917
Kohlenhydrate und Hyperlipidämien 2308

Kohlenmonoxid, Diagnostik 1692

Kollumkarzinom, Vorsorgeuntersuchung 2452

Koma hepaticum, Therapie 1830

- bei Leberleiden 2237

Komplementanalysen, quantitative $\left(C^{\prime}: 3\right) \mathbf{1 9 7 8}$

Konservenplasma, grünes 2706

Kontaktallergie und Nifurprazin 1685

Kontaktmangel und Hydergin 2434

Kontrastmittel 2704*

Kontrastmittelkinetik 2021

Konzentrationsfähigkeit, Hydergin 2433

Koproporphyrin im Magensaft 2392

Koronararterienstenosen, Revaskularisation 1903

Koronar-Cine-Angiographie 1904

Koronarinfarkt und Schock 1642

Korcnarinsuffizienz und Herzmuskelnekrose $\mathbf{1 8 5 1}$

Koronarkrankheit, Studie in Framingham 1667

Koronarreserve des Menschen 2563

Kostensicherung $2523 \mathrm{R}$

Krämpfe, postvakzinale 2403

Kraftfahrer und Blendschutzbrille 1545

Krankenernährung 2360*

Krankenhäuser, Personallage 1955

Krankenhaus und Insektizide 2126

Krankenhauspatienten, Zahl 1588

Krankenschwestern, Lehrbuch 1708*

Krankenunterlagen, Rückgabe $1947 \mathrm{R}$

-, Verfügungsberechtigung $2357 \mathrm{R}$

Krankheiten im Altertum 2185*

-, Früherkennung 1844:

-, Meldung an Gesundheitsämter $1767 \mathrm{R}$

-, seltene und Auskunft 2067

Krebsvorsorgeuntersuchungen, Effektivität 2015, 2452, 2569

Krebsvorstellungen der Bevölkerung $\mathbf{1 5 8 1}$

Krehl, Preis 19691669

- Preisausschreibungen 1669

Kreislauf und CSN-Stimulator 1719

-, Stromzeitvolumina 1621

Kreislaufregulationsstörung, hypotone $\mathbf{2 1 7 2}$

Kriegsopferfürsorge, Kosten 2302

Kryochirurgie in der Urologie 1497

Kryopräzipitate, antihämophile 2541

Kryopräzipitatherstellung 1799

Kryptorchismus, Gonadotropine zu Diabetes? 1626

Kürettennadel nach Ramel zur Pleurabiopsie $\mathbf{1 7 2 5}$

Kuhmilch und Lysozym 1459

L

Labadie und Tiffenau-Werte bei Asthma 1811

Laboratoriumsbefunde be Paraproteinämien $\mathbf{2 5 5 8}$

Lactatdehydrogenase, Bedeutung 1882

Lähmungen beim Zoster 1861

Laidlaw-Syndrom 1445

Landbevölkerung und Arteriosklerose 2575

Langenbeck-Preis 19701777

Laxantien, chronischer Gebrauch 1506

Laporoskopie, Atlas 2460:

- und Hepatitis und Zirrhose 1633

LDH, Bedeutung 1882

Leber und Arteriographie des Oberbauches 2396

- und Eisenstoffwechsel 1841

- und Endokrinium 1627*

-, Erkrankungen 2459:

-, Fettleber und Therapie $\mathbf{1 6 1 9}$

- und Glucocorticoide, Proteinsynthese $\mathbf{1 6 1 5}$

- und Glykogenose 1420

- und Hitzschlag 1696

- und LDH 1882

- und Polyarthritis chronica 2645

- und Vitamin D 1998

Leberbiopsie, Aufklärung 1463

- bei Hepatitis und Zirrhose 1634

- bei Porphyria cutanea tarda 1578

Leberdiagnostik 1466

Leberdystrophie und $\gamma-\mathrm{GT}$ Aktivität 1911

Leberentzündung nach Fettemulsionen $\mathbf{1 8 2 5}$

Lebererkrankung und Serumtransaminasen 2251

Leberfunktion und Alkoholismus, Xyloseresorption 1533

Leberkoma, Kreuzzirkulation mit Pavianen 1776

- und Osophagusvarizenblutung $\mathbf{1 4 7 8}$

-, Therapie 1830, 2235

Leberkrankheiten, Blutsenkungs- 


\section{- und LDH 1884}

- nach Magenresektionen 2167

- und Narbenleber 1637

- und Ósophagusvarizen-

blutung 1476

-, posthepatitische 1838

- und Xyloseresorption 2297

Legasthenie und Ganzwortmethode 1462, 1893

Leichenbeschauer, Anzeigepflicht 2570

Lendle, L. † 2455

Lepra in New York 2130

Letterer-Siwe und Lymphknoten 2199

Leucin-Aminopeptidase und Glutamat-Transpeptidase 1911

L.eukämie, akute und Zytochemie 2320

- und L-Asparaginase 1669

- durch Benzol 1665, 1703

- und Erythroleukämie 2419

- nach Extremitätenamputation 2571

- des Kindes, Dauno-Rubidomycin 1972

- bei Kindern, Therapie 1993

-, Lymphknoten 2199

-, Pseudo-Pelger-Zellen 2284

- und Röntgenstrahlen 1425

Leukosen und Pneumocystis carinii 2137

- und Retraktion 1738

- unter Tolbutamid? 1625

Leukosenviren und Impfstoffe 1660

Leukozidin und Staphylokokken 2351

Leukozyten und Daunoblastinbehandlung 1974

Leukozytenphosphatase, alkalische und Leukämie $\mathbf{2 3 2 1}$

Leukozytenprovokation mit Echinacin-Test 1406

Leukozyturie in der Schwangerschaft 1554

Libyen, Länderkunde 2461*

Liddle-Syndrom 1445

Lipase in Arzneien 1870

- im Serum und Pankreasstimulation 2086

Lipasen der Staphylokokken 2352

Lipide, Steigerung durch Ernährung 2307

Lipiodol, Leberspeicherung 2547

Lipoidnephrose und Nierenbiopsie $\mathbf{1 6 7 4}$

Lipoproteinelektrophorese bei M. Marfan 2284

Lippen, Krankheiten $1775^{*}$

Liquordrainage bei Hirntumoren 1418

Liquorelektrolyte und nephrogene Enzephalopathie 1743

Lithiumintoxikation 2516

Lithium-Therapie 2512

- bei Psychosen 2659

Lithotrypsie bei Harnstein $\mathbf{2 3 8 3}$

Luesserologie und Pockenimpfung 2186

Luizym, Aktivitäten 1871

Lumpy Skin-Virus 2661*
Lunge des Bergmannes 2375

- und Goodpasture-Syndrom 1820

- und Herpangina-Epidemie 1961

- und Hühnerzüchtung,

Pneumopathie 2627

- und LDH 1882

- und Lymphangiektasien 1987

- und Nitrofurantoinfieber 1922

- und Pneumocystis carinii-

Pneumonie 2136

- und Polyarthritis chronica 2644

-, transpulmonale Fistel 1917

Lungenarterienembolie nach Venae sectio 1796

Lungenbefunde bei Sepsis 2226

Lungenbiopsie, Bedeutung 1724

Lungenembolie und Psychose 2181

-, Thombolyse 2293

Lungenerkrankungen und LDH 1883

- durch Uberempfindlichkeit $2460 *$

L.ungenfibrose, Ätiologie und Diagnostik 2290

Lungenfunktion und Asthma 1805

Lungenfunktionsprüfung 2703

-, Normalwerte 1785

Lungenfunktionsstörungen, Begutachtung 1843*

Lungenhämosiderose, Immunsuppression 1813

Lungeninfarkt, Diagnostik 2605

Lungenkrankheiten und

Entwicklungsanomalien 2185*

Lungenkrebs, Prävention 2015

- und Röntgenreihenuntersuchung 2180

-, Therapie 1559

Lungenmycetom 2045

Lungenmykosen, Therapie 1992

Lungenproteinose, alveoläre 2438

Lungenschlagadersarkome $\mathbf{1 6 0 9}$

Lungenszintigraphie 2606

Lungentuberkulose 1952*

Lungentumoren, Biopsie 1726

Lungenübersichtsaufnahme, Verbesserung 1484

Lupus erythematodes, immunsuppressive Therapie $\mathbf{2 4 4 7}$

- Syndrom bei Procainamid 2338

Lupus miliaris und rosceaartige Dermatitis $\mathbf{1 3 9 6}$

Lyell-Syndrom 1422

Lymphadenitiden, unspezifische 2195

Lymphangiektasien, angeborene pulmonale $\mathbf{1 9 8 7}$

Lymphknoten und LDH $\mathbf{1 8 8 2}$

Lymphknotenerkrankungen in Schleswig-Holstein $\mathbf{2 1 9 4}$

Lymphoblastenleukämie, L-Asparaginase 1669

Lymphogranulomatose, Cyclophosphamid 2583

Lymphogranulom und Pneumocystis carinii 2137

Lymphographie und Lipiodolspeicherung der Leber 2547

Lymphosarkom und Cyclophosphamid $\mathbf{2 5 8 8}$

Lymphozyten und Azathioprin 2269

-, fetale und Geschlechtsdiagnose 2663
Lymphozytenreaktionen bei Morbus Hodgkin 1867

Lysismuster von Staphylokokken 1448

Lysozym, enterales bei Säugling 1458

- der Staphylokokken 2352

M

Macula densa, Theorie 2178

Madaus, Betriebsjubiläum 2362

Maffucci-Syndrom 1991

Mafu Strip 40, Bedenken? 2126

Magen, Chirurgie 2064"

- Frühkarzinom, Gastrokamera 2440

-, Tonus und Motilität nach Vagotomie und Pyloroplastik 1513

Magenblutung, Bauchdeckenerschlaffung 2241

Magenchirurgie, neuzeitliche 1506

Magengeschwür, Betazoltest 2278

- und Leberzirrhose 2167

-, Vagotomie 1516

Magenresektionen und Leberzirrhose $\mathbf{2 1 6 7}$

-, Pankreasfermentersat $\% 1872$

Magensaft, Porphyrine $\mathbf{2 3 9 2}$

Magensaftuntersuchung 1660 , 2060

Magensalzsäure und Eisenresorption 1.503

Magensekretion und Betazoltest 2278

- und Endoradiosonde 2274

Magnesiumsulfat, Schädlichkeit? 1506

Makroglobulin und Pneumokokkenmeningitis $\mathbf{2 1 5 0}$

Makrophagenresistenz, erworbene 2355

Malabsorption und Coeliakie 1856

Malabsorptionssyndrom bei Immunglobulinmangel 2023

-, Katamnesen 2210

Malaria als Importinfektion 1518

Mammakarzinom und Cyclophosphamid $\mathbf{2 5 8 8}$

-, Frühdiagnostik $1507^{*}$

-, Prävention 2015

-, Schutzmechanismen $\mathbf{2 4 6 7}$

Mammaphantome 2368

Mammaria-Implantation der

Koronarien 1906

Mammographie, 2015

Mangelernährung, intrauterine und Hirnveränderungen 2130

Manie, Lithiumbehandlung 2513

Mannit und Furosemid bei Niereninsuffizienz 1568

Marburg-Virus und Impfstoffe 1661

Marfan-Syndrom, Elektrophorese und Immunologie 2284

- - Methandrostenolon 1588

Markaplasie unter Daunoblastin 1977

Markdestruktionen bei

Meningitis $\mathbf{1 5 2 9}$

Martini-Preis 2530

Martini-Stiftung 19701777

McArdle Glykogenose 1421

Medikamente und Erythematodes-Auslösung 2041

Medizin, Buchillustration 2253*

- Farbfotographie 1549

-, innere und Glucocorticoide $1427 *$

- und Länderkunde, Libyen 2461*

-, Nobelpreis 2302, 2405

- perinatale 2252

Medizinalassistenten, Begutachtung $2243 \mathrm{R}$

Mediziner und Physik 1548"

Medizinstudenten, Rauchgewohnheiten 1549

Megaloblastenanämie, Psychosen 1474

Melanom, Lymphknotenmetastasen 2202

Melioidose 1509

Membranpermeabilität der Erythrozyten 2360

Menghini Punktion bei Hepatitis 1636

Menopause und Endometrium und Mamma 2470

Meningitis, eitrige und Markdestruktion $\mathbf{1 5 2 9}$

- durch Enteroviren 2071

- bei Herpangina 1964

- nach Mumps 1599

- bei Mumps und Bettruhe 2004

- durch Pneumokokken, Neuraminidase 2149

- durch Vibrio fetus 2400, 2484

Meningopathia leucaemica 1995

Mensch, Koronarreserve 2563

Menschen, Versuche 2528*

Menschenwürde und Arztgeheimnis $2060 \mathrm{R}$

Mephentermin und Phonokardiogramm-Diagnostik 1751

Mercaptopurin und Erythroleukämic $\mathbf{2 4 2 0}$

-, immunsupprcssive Therapie 2444

- bei Leukämie 1993

- bei Lungenhämosiderose 1813

Merck-Preis 19691669

Mercuribenzoat und Tollwutvirus 1702

Mesenchymstoffwechse! und Vitamin D 1878

Mesenterialgefäßverschluß, akuter 1937 
Metyrapon und Hyperaldosteronismus 2052

Mexase, Aktivität 1871

Miescher, DNS-Entdeckung 1948

Mikrochirurgie in der Hirnchirurgie 1496

Mikrofraktographie $\mathbf{1 8 3 7}$

Mikrobiologie 1628

-, medizinische $2184^{*}$

Mikroskopie, Entwicklung 1832

Milch- und Lysozymzusatz 1462

Milchdrüse und Oxytocin 2613

Milz und LDH 1882

- und Polyarthritis 2645

Mineralocorticoidsyndrom 1442

Mineralsalzbestimmung am

kindlichen Skelett 1983

Minkowski-Preis 19692130

Mitomycin, immunsuppressive Therapie $\mathbf{2 4 4 4}$

Mitralstenose und Anti-

koagulantientherapie 1424

Mondgestein, Untersuchung 2663

Mongolismus und Chromosomenuntersuchungen $\mathbf{2 6 7 6}$

Monika-Stiftung 1899

Monoamine und Parkinson 1942

Morbus Bechterew, Röntgentherapie 1505

- Conn 1442

- Cushing und Alkalose 1445

- Di Guglielmo des Erwachsenen 2421

- haemolyticus neonatorum, Prophylaxe 1437, 1654, 1655

- -, Fruchtwasser-Bilirubin 2602

Morbus Hodgkin, Lymphknotenbeteiligung 2199

- - und Lymphozytenreaktionen 1867

- Parkinson, Therapie 2678

- Waldenström, C'3-Gehalt 1980

- Wilson 1628:

Moronal bei Mykosen 1992

Morphium und Herzschock 1753

Morquiosche Krankheit, Mineralsalzbestimmung 1986

Mosaike bei Anstaltspatienten 2675

Moschcowitz-Syndrom 2326

Mucoviscidose, C'3-Gehalt 1980

-, Diagnose 1677

Mumpsinfektion und EndokardFibroelastose 2529

Mumpsmeningitis, Bedeutung 1599

-, Desinfektion 1585

-, Verhütung 2004

Mundhöhlenkarzinom, Therapie $1667^{*}$

Mundschleimhaut, Krankheiten $1775^{\circ}$

Muskeldurchblutung, Steigerung 2348

Muskelparesen bei Zoster 1861

Mutagenitätsprüfung $2362^{*}$

Mutationsproblem beim

Menschen $2183^{*}$

Mycetom der Lunge 2045

Myelitis und Enteroviren 2071

- bei Mumps 1600

Myelofibrose und Ösophagusvarizen 2163

Myelogramm bei Di-GuglielmoSyndrom 2416

Myelographie 2704:

Myelom und Cyclophosphamid 2587

-, neues Zytostatikum 2495
Myeloproliferation und Hämoglobinurie 1891

Myelosen und Pseudo-PelgerZellen 2284

- und Thrombozytenfunktion 1738

Mykosen, Allergie 2508

- der Lunge 1992

Myokard, Revaskularisation 1903

Myokardinfarkt 2646

- und Schock 1640

Myokardiopathien 1755

-, hypertrophierende obstruktive 2579

Myokarditis $\mathbf{1 7 5 5}$

- und Herzrhythmus-

störungen 2672

- und LDH 1983

Myopathien und LDH 1884

Myxödem und Novothyral 2482

Myxoviren und Impfstoffe $\mathbf{1 6 5 9}$

- und Tollwutvirus 1699

$\mathrm{N}$

Na-Desoxycholat und Tollwutvirus 1701

$\mathrm{Na}$,-EDTA u. Tollwutvirus $\mathbf{1 7 0 1}$

Nachtdienst in Kliniken 2298

Nägel, Erkrankungen $1508^{*}$

Nahrungsmittel u. Vitamin D 1997

Nalidixinsäure u. Nitrofurantoin 1428

- bei Pyelitis 1939

Narbenleber, postnekrotische 1637

Narcotin, Wirkung 1585

Narkose u. Octapressin bei portocavalem Shunt $\mathbf{1 6 7 9}$

Narrenschelle bei Mycetomen der Lunge 2048

Nasenheilkunde $2183^{*}$

Natrium bei Niereninsuffizienz 2114

- im Schweiß u. Mucoviscidose 1677

Natriumausscheidung $\mathrm{u}$.

Niereninsuffizienz 2265

Natriumbicarbonat bei renaler Acidose 1398

Natriumbilanz bei renaler Acidose $\mathbf{1 4 0 0}$

Natriumsekretion u. Diuretikatherapie 1572

Natriumfluorid zur OsteoporoseTherapie $\mathbf{1 7 3 0}$

Natriumhaushalt u. Aldosteronismus 2051 orscher Leopoldina, Ehrenmitglieder 2411

_ -, Mitgliedswahl 1428

Nebennieren u. LDH 1882

Nebennierenrinde u. Corticoide 2488

Nebenschilddrüsenadenom nach Nierentransplantation $\mathbf{2 1 4 0}$

Neomycin u. Nocardiose 2506

Nephrektomie u. Blutdruck 2083

-, erste 2247

Nephritis bei Herpangina 1964

u. Immunsuppressiva $\mathbf{2 4 4 7}$

Nephroblastome des Neugeborenen 2598

Nephrolithiasis u. Sitzberuf $\mathbf{1 6 6 6}$

Nephrologie im Kindesalter $2621^{*}$
Nephropathie durch CoxsackieViren 2302

- u. Goodpasture-Syndrom 2637

Nephrose u. Nierenbiopsie $\mathbf{1 6 7 3}$

Nepresol u. Glomerulumfiltrat 1688

Nervenkrankheiten und Schweigepflicht $1767 \mathrm{R}$

-, Therapie 1546:

Nervensystem, zentrales u. Amputation 2373

Nesidioblastose 1709

Netzhaut, Fluoreszenzangiographie 1587

- u. Ovulationshemmer $\mathbf{1 7 6 2}$

Neugeborene u. Bauchlage 2449

-, Hämoglobinresorption 1950

-, Immunglobulin-anti-D-Gabe 1438

-, Lymphangiektasien der Lunge 1987

- u. Nephroblastome 2598

-, neurologische Untersuchung 2128

-, perinatale Medizin 2252*

- u. Vibrio fetus $\mathbf{2 4 8 6}$

- u. Zytomegalie 1969

Neuraminidase u. Pneumokokken-Infektion $\mathbf{2 1 4 9}$

Neurochirurgie $2410^{*}$

-, Fortschritte $2361^{*}, 2622^{*}$

Neurohypophyse $1773^{*}$

Neurologie $1897^{*}$

-, pädiatrische $2128^{*}$

Neurolues, Therapie 1486

Neuropathie, paraneoplastische 2103

Neuroplegika als Symptholytika 1941

Neurose des Herzens $1773^{\text {* }}$

New-Peterson-Syndrom 1445

New York, Aussätzige 2130

Niere u. Acidose, Therapie 1398

- u. Bilivistan 2019

-, Enzephalopathie u. Elektrolyte 1743

- u. Goodpasture-Syndrom 1820

- u. Herzbeteiligung 1759

-, juxtaglomerulärer Apparat 2173

- u. LDH 1882

- u. Peritonealdialyse 2059

- u. Polyarthritis 2645

- u. Reflux 2054

- u. Vitamin D 1879

Nierenarterienstenose, Alkalose 1443

-, Reninaktivität $\mathbf{1 4 1 7}$

Nierenbiopsie u. Nephrose 1673

Nierenblutung bei Hämophilie A, Therapie 2543

Nierendurchblutung $\mathrm{u}$. Dihydralazin 1688

Nierenerkrankungen $u$. Kalium verlust $\mathbf{2 6 3 5}$

Niereninffizienz u. Bananengenuß 1765

-, Diätetik 2112

- u. Diuretikatherapie 1568

- bei Malarie 1520

- u. Plasma-Renin 2262

- u. Quinton-Shunt 1604

- bei Tetrachlorkohlenstoffvergiftung 1646

Nierenkranker u. Heimdialyse 1888

Nierenkrankheiten $1843^{*}$

-, Diätetik 2252*

- u. LDH 1884

Nierensteine u. Hitzeberuf 1666 - nach PAS-Calcium 1491

-, Therapie 2382

Nierentransplantation,

Hyperparathyreoidismus $\mathbf{2 1 4 0}$

- u. LDH 1884

- u. Lungeninfiltration 2202

-, Vorbehandlung 2082

Nierenversagen bei Hitzschlag 1966

- u. Hypercalcämie 2155

Nifurprazin u. Allergie $\mathbf{1 6 8 5}$

Nikotin 1951

Nitrofurantoin-Fieber 1922, 2523

- u. Nalidixinsäure 1428

- bei Pyelitis 1939

- u. Schwangerschaftsbakteriurie 1555

Nobelpreis für Medizin 2302, 2405

Nocardiose, Antibiotikatherapie 2505

Nomenklatur u. Andrologie 2530

Noradrenalin u. Parkinson 1942

- u. PhonokardiogrammDiagnostik 1751

Norethisteronönanthat,

Kontrazeptivum 2473

Notdienst, ärztlicher $2005 \mathrm{R}$

Notfalldienst, Mißbrauch 2061

Novocamidbehandlung, Lupus-erythematodes-Syndrom 2338

Novothyral, Wirksamkeit 2478

Nuclease der Staphylokokken 2352

Nutrizym, Aktivität 1871

Nystatin bei Mykosen 1992

\section{$\mathrm{O}$}

Oberbaucharteriographie 2395

Oberschenkelamputation $\mathrm{u}$.

Uberlastung 1626

Octapressin bei Osophagusvarizenblutung 2063

- u. Pfortaderdruck 1679

Odeme bei Antiepileptika? 1896

Oehlecker-Preis 1470

Osophagus, Bildband 2064*

- u. Herzstillstand 2191

Osophagusvarizen u. Myelofibrose $\mathbf{2 1 6 3}$

Osophagusvarizenblutung, akute 
Organkonservierung 2083

Organphosphat-Inhibitoren 2695

Organtransplantationen, Nachbehandlung $\mathbf{2 0 8 2}$

Oropharynxkarzinom, Therapie 1668 *

Orthopädie, Operationsverfahren $2705^{*}$

Orthostasereaktion, Belastungselektrokardiogramm 2182

Osmotherapie bei Intoxikationen 1896

Osteogenesis imperfecta u. Mineralsalzbestimmung $\mathbf{1 9 8 6}$

Osteopathie nach Heparinbehandlung $\mathbf{2 3 3 4}$

Osteoporose u. Mineralsalzbestimmung 1986

-, Therapie $\mathbf{1 7 3 0}$

Osteotomien, Redondrainage 2359

Oswald-Schmiedeberg-Plakette 1630

Otitis media, Schichtuntersuchung 2704*

Otosklerose, Operation 1773*

Ovarialinsuffizienz u.

Endometrium 2470

Ovulationshemmer 1897"

-, Augenwirkung 1761

- u. Endometriumkarzinome 2469, 2471

-, klinisch-chemische Normalwerte 2522

Ovulationshemmer und Konservenplasma 2706

Oxacillin 2608

Oxyprenolol 2560

Oxytocin u. Vasopressin 261

\section{P}

PAH-Clearance und ${ }^{131} \mathrm{~J}$-o-Jodhippursäure-Clearance $\mathbf{2 6 8 7}$

Pankreas u. LDH 1882

-, Pharmakoangiographie 1970

- u. Tetrachlorkohlenstoffvergiftung $\mathbf{1 6 5 0}$

Pankreasfermente, enzymatische Aktivität $\mathbf{1 8 7 0}$

Pankrcasinsuffizienz, Untersuchungen 2091

Pankreaskarzinom, Immunologie u. Diagnostik 2411

Pankreatan, Aktivitäten $\mathbf{1 8 7 1}$

Pankreozymin und Serumlipase 2086

Pankrodigest, Aktivität 1871

Panmyelophthise u. Pneumocystis carinii 2137

Panzytopenie nach Imurel $\mathbf{2 2 7 0}$

Papierelektrophorese u. MarfanSyndrom 2289

Papilla Vaterie, Endoskopie 2575

Papillenschwellung nach Ovulationshemmer $\mathbf{1 7 6 2}$

Papovaviren u. Impfstoffe $\mathbf{1 6 6 0}$

Paraleukoblasten, Zytochemie 2320

Paraleukoblastenleukämie, Zellmorphologie und Prognose 2550

Paralymphoblasten-Leukämie 2320

Paralyse, progressive u. Fieberu. Penicillintherapie $\mathbf{1 4 8 6}$

Paramyeloblasten-Leukämie $\mathbf{2 3 2 0}$
Parapromyelozyten-Leukämie 2320

Paraproteinämie, Cytostatikum 2495

-, Laboratoriumsbefunde 2558

- u. Lymphknoten 2199

Parkinsonsche Krankheit 1426 *

-, Amantadin 1776

- -, L-Dopa-Therapie 2670

- - durch Virusinfektion? 2525

Parkinsonismus durch Psychopharmaka 1941

PAS-Calcium u. Urolithiasis 1491, 1928

Pasteurella multocida bei Tierbissen 1816

Patch-Test, Wert 1677

Pathologie für Chirurgen 1708*

- des Herzens u. der Gefäße $2622^{*}$

-, klinische des Schwachsinn $1774 *$

-, Thrombose und Arteriosklerose 2662

Pathophysiologie des Diabetes mellitus 2301*

Paviane, Kreuzzirkulation beim Leberkoma 1776

$\mathrm{PB}{ }^{127}$ u u. Schilddrüse $\mathbf{2 3 8 6}$

Pelger-Huetsche Kernanomalie 2284

Penicillin bei Rheumatismus 2007

Penicilline, halbsynthetische $u$. Indikation 2607

Penicillintherapie der Paralyse 1486

Penisphantome 2368

Pentamidine bei Pneumocystis carinii 2139

Pepsaldra, Aktivitäten 1871

Peptidasen in Arzneien 1870

Periarthritis humeroscapularis, Therapie 1579

Perikarderguß, chronischer idiopathischer $\mathbf{2 1 6 0}$

Peritonealdialyse, Flüssigkeitsbilanz 1873, 2959

- bei Thyreotoxikose 2615

Perniciosa-Therapie 2356

Persantin u. Koronarien 2564

Persönlichkeitsbildung u. Fachkongreß $1704 \mathrm{R}$

Personallage der Krankenhäuser 1955

Perspiration insensibilis be Peritonealdialyse $\mathbf{1 8 7 3}$

Pertussis u. Ampicillin 2435

-, Klimakammer 1624

Pest, Gefahr 2530

Pfeiffersches Drüsenfieber u. Lymphadenitis $\mathbf{2 1 9 6}$

Pflegesatz u. Belegarzt 2618

Pfortaderdruck u. Octapressin 1679

pH-Endoradiosonde 2274

Phäochromozytom u. Alkalose 1444

-, Blutdrucklabilität 2299

- u. Rezeptorenblocker 2561

Phantome nach Amputation 2367

Pharmaka, gefäßwirksame bei Herzschock 1753

- zur PhonokardiographieDiagnostik 1750

Pharmakoangiographie des Pankreas 1970

Pharmakologie, Grundlagen 1952*

Pharmakotherapie, Einführung 2662

Phasenkontrastverfahren 1833
Phenethicillin 2608

Phenylephrin u. Phonokardiogramm-Diagnostik $\mathbf{1 7 5 1}$

Phlebologie, Gesellschaft 2187

Phonokardiographie, pharmakodynamische $\mathbf{1 7 5 0}$

Phosphatase u. $\gamma$-GlutamylTranspeptidase 1911

Phosphattherapie der Harnsteine 2385

Phospholipase u. Tollwutvirus 1701

Physiologie, medizinische 1548*

-, Nobelpreis 2405

Physik für Mediziner 1548*

Phytohämagglutinin u. HodgkinLymphozyten 1867

Picomaviren u. Impfstoffe $\mathbf{1 6 5 9}$

Pigmentzirrhose u. Magenresektion $\mathbf{2 1 6 9}$

Pilokarpin bei Asthmatikern 1651

- Iontophorese bei Mucoviscidose 1677

Pilze u. Lunge, Allergien 2460*

Pilzerkrankung der Lunge 2046

Pilzsporen, Allergie 2508

Pimaricin bei Lungenmykosen 1992

Piringersche Lymphadenitis $\mathbf{2 1 9 8}$

Plasmafluß, renaler 2685

Plasmapherese bei Hämophilie B 2145

Plasma-Renin u. Niereninsuffizienz 2262

Plasmazytom, C' ${ }_{3}$-Gehalt 1980

- u. Pneumocystis carinii 2137

- u. Thrombozytenfunktion $\mathbf{1 7 3 8}$

Plattenepithelkarzinom, Bleomycin 2250

Plethysmographie u. Lungenfunktion-Normalwerte $\mathbf{1 7 8 5}$

Pleurabiopsie, Bedeutung 1724

Pleuraverschwartung, Biopsie 1724

Plexusläsionen u. Phantome 2368

Pneumocystis carinii u. Nierentransplantation 2202

- -, Pneumonie 2135

Pneumokokkeninfektionen u. Neuraminidase $\mathbf{2 1 4 9}$

Pneumokoniose des Bergmanns 2375

Pneumoektomie u. Bronchialkarzinom 1561

Pneumonie durch Pneumocystis carinii $\mathbf{2 1 3 5}$

Pneumopathie, allergischinterstitielle 2629

Pocken, Frühimpfung? 2403

Pockenimpfung u. Luesserologie 2186

Pockenviren der Geflügel u. Impfstoffe $\mathbf{1 6 5 9}$

Podagra, Röntgenbefund 1909

Podaphyllin, immun-suppressive Therapie 2444

Polarisationsmikroskopie 1833

Poliklinik, Funktion u. Aufbau 1933

Polyäthylenglykol u. Allergie 1685

Polyarthritis, chronische $2065^{*}$

-, Goldtherapic u. Erythematodes 2040

- u. innere Organbeteiligung 2644

-, primärchronische u. Immunsuppressiva 2447

Polychondritis mit Aortenaneurysma 2033

Polymyalgia rheumatica 2232

Polyneuropathie, sensible bei

Thymuskarzinom 2102

Polypeptide u. Neurohypophyse $1774^{*}$

Polyradikuloneuritis durch Enteroviren 2071

Polyurie u. Hypercalcurie 2155

Polyzythämiker als Blutspender 2250

Pompesche Krankheit 1414, 1420

Ponndorf-Impfung 1772

Porphyria cutanea tarda, Diagnose 1577

Porphyrie u. Ovulatiomshemmer 2522

Porphyrine in Magen- $u$. Duodenalsaft 2392

Präparationsmethoden der Raster-Elektronenmikroskopie 1836

Präventivmedizin, Preis 2707

Prednisolon, Hemmwirkung 2491

- - bei Leukämie 2325

Prednison bei Erythroleukämie 2420

- bei Hepatitis 2700

- bei Leukämie 1993

- u. Lungenhämosiderose 1813

- u. Osteopathie 2336

Preisausschreibung 1428, 1470 , $1549,1669,1709,1777,2011$ 2067,2255, 2707

Preise für Tierquälerei 1777

Preisverleihungen 1469, 1509, $1588,16,30,1669,1845,1599$, 2011, 2130, 2254, 2362, 2411, 2463, 2530, 2575, 2707

Presinol u. Blutdruck 2083

Price-Jones-Kurve unter Imurel 2272

Primogonyl zu Diabetes? 1626

Privatpatienten, Gebühren 2526

Privatversicherer, Auskunftspflicht 2242 R

Probethorakotomie u. Strahlentherapie des Bronchialkarzinom 1546

Procainamid, Lupus erythematodes-Syndrom 2338

Profenazin, Wirkung 1943

Proktologie, Gesellschaft 2187 
Pseudohermaphroditismus femininus bei AGS-Syndrom 2229

Pseudomonas aeruginosa und Carbenicillin 2501

Pseudo-Pelger-Zellen bei Leukämie 2284

Pseudotuberkulose, Lymphadenitis 2197

Pseudotumor cerebri nach Ovulationshemmern 1762

- bei Hämophilie B 2145

Psoriasis des Kopfes 1466

- u. rosoceaartige Dermatitis 1395

Psychiatrie, Therapieform 1668*

Psychoanalyse bei Schizophrenen 2460"

Psychodiagnostik 2409*

Psychologie der Amputierten 2369

Psychopharmaka u. Analgetika 2528"

- u. Parkinsonismus 1941

- u. Psychotherapie 2184

Psychosen bei Bizz-Avitaminosen 1473

-, Lithiumtherapie 2514, 2654

- u. Lungenembolie 2181

Psychotherapie 2409*

- u. Psychopharmaka 2184.

Pulmocath, Einschwemmkatheter 2345

Pulmonalarteriendruck u. CSNReizung 1719

Purinethol bei Leukämie 1993 , 2325

Purpura, thrombotisch-thrombozytopenische 2326

Pyämie, Lungenbefunde $\mathbf{2 2 2 6}$

Pyelonephritis, Keimbestimmung 2223

- im Kindesalter 1939

- u. Plasmarenin 2262

- u. Refluxe 2056

- Therapie im Kindesalter 2356

Pyloroplastik u. Mobilität u.

Tonus 1513

Querschnittslähmung u. Phantome 2368

Quick-Test u. Thrombotest 2525

Quick-Wert u. Wunddehiszenzen 1405

Quinton-Shunt bei Hämodialyse 1604

$\mathbf{R}$

Rabiesviren u. Impfstoffe $\mathbf{1 6 6 0}$

Rachitis, Mineralsalzbestimmung 1985

- u. Vitamin D 1997

Radargerät, Stress 1845

Radiojoddiagnostik u. Hyperthyreose $\mathbf{2 6 3 1}$

Radiologie 2183*

-, medizinische 2704"

Radionephrographie 2686

Radiostrontium, Skeletszintigraphie $2704^{*}$

Raster-Elektronenmikroskopie 1832
Rauchen u. Gesundheit 1509

- u. Herzinfarkt 1667

- bei Medizinstudenten 1549

Rauwolff, L. u. Horoskop 2245

Recetan $\mathbf{2 5 6 0}$

Rechtsherzinsuffizienz, Digitalisresorption 2359

Redondrainage nach Osteotomien 2359

Reflux, vesikouretraler 2053

Regresspflicht des Amtsarztes $2656 \mathrm{R}$

Rektumblase $\mathbf{2 1 1 6}$

Renin u. Hyperaldosteronismus 2050

- u. Niereninsuffizienz 2262

-, Wirkung 2177

Reninaktivität im Blut, Bestimmung 1417

Reoviren u. Impfstoffe $\mathbf{1 6 6 0}$

Reparil bei Harnstein $\mathbf{2 3 8 2}$

Reserpin u. Parkinsonismus 1942

Residualvolumen, Normalwerte 1705

Resochin gegen Amyloid 2692

- bei Hepatitis 2299

Rest-N u. Diuretikatherapie 1573

- - u. Nierenversagen 2264

Retikulosen u. Lymphknoten 2201

Retraktion der Blutplättchen 1735

B-Rezeptorenblocker, Behandlung 2560

Rh-Inkompatibilität u. Fruchtwasser-Bilirubin 2602

Rhabdoviren u. Tollwutvirus 1697

Rhesus-Desensibilisierung 1654, 1655

Rhesusfaktor u. Prophylaxe $\mathbf{1 4 3 3}$

Rheumatismus 2007

-, Arthritis u. Operation 2009*

-, chronischer 2065*

-, Probleme 1468:

Rhinoviren 1843"

Rhodanin gegen Virusinfektione!n 1541

Rhythmusstörungen u. d-Propranolol 2669

- u. Rezeptorenblocker 2561

Ribonuclease, Aminosäuresequenz 2695

Ribonucleinsäure, Basensequenz 2254

- Kinetik u. Phytohämagglutinin 1867

Rifampicin bei Viruskrankheiten 1954

Rinderpestvirus 2661*

Ring-Stripper nach Vollmar 2317

Rippentumor u. Pleurabiopsie 1724

RNS u. Virusinfektion 1629

Röntgenaufnahmen, Verbesserung 1483

Röntgenbefunde bei Gicht 1909

Röntgenbestrahlung der Hypophysentumoren $\mathbf{2 4 4 3}$

Röntgenbild des Lungeninfarkts 2606

Röntgendiagnostik des Skelet 2183:

Röntgenkontrastmittel u. Hyperthyreose 2631

Röntgenkristallographie der Enzyme 2698

Röntgen-Lungenbefunde bei Sepsis 2226
Röntgenreihenuntersuchung $\mathrm{u}$. Lungenkrebs 2180

- in der Schwangerschaft 2117

Röntgenstrahlen, Katarakt 1585

- u. Leukämie 1425

Röntgentherapie des M. Bechterew 1505

Röntgen-Thoraxdiagnostik, programmiert 2021*

Röteln u. Lymphadenitis 2146

-, Schutzimpfung 1626

Rollenpumpe bei Peritonealdialyse $\mathbf{1 8 7 6}$

Rosacea u. Dermatitis perioralis 1393

Rubidomycin bei Leukämie 1993

Rückenlage beim Säugling $\mathbf{2 4 5 0}$

Rückrechnung bei Blutalkohol-

bestimmung 2259

\section{S}

Säuglinge u. Bauchlage 2449

- u. Borsäure 1841

- u. Candida albicans 2254

- u. Lysozym 1458

- u. Lysozym der Frauenmilch 1459

-, Mineralsalzbestimmung am Knochen 1985

- u. Wilms-Tumoren 2600

Säuglingsenteritis u. Lysozym 1460

Salmonellen u. Ampicillin 2610

Sanokrysin u. Auge 2389

Sarkoidose, Lymphadenitis $\mathbf{2 1 9 7}$

Sarkom und Heparin 2706

Sarkome der Arteria pulmonalis 1607

- lymphoretikuläre 2199

Sauerstoff u. Herzschock 1753

Sauerstoffdruck, arterieller bei Bergleuten 2376

Sauerstoffmangel u. Herzmuskelnekrosen $\mathbf{1 8 5 2}$

Sauerstofftransport in Blut u. Gewebe 1707"

Schädeltrauma u. Hirnblutung 1617

Schilddrüse u. Jod 2386

- u. Jodzufuhr 2632

- u. LDH 1882

Schilddrüsendiagnostik, u. JodZahnpasten 2408

Schilddrüsenhormone, Darreichung 2478

- u. Trijodthyronin-Test $\mathbf{1 4 5 5}$

Schimmelpilz u. Allergie $\mathbf{2 5 0 8}$

Schizophrene, Psychoanalyse $2460 *$

Schizophrenie u. Chromosomen 2676

- Therapie 2128

Schlafstörung u. Hydergin 2432

Schleswig-Holstein, Lymphknotenerkrankungen $\mathbf{2 1 9 4}$

Schlingenextraktion des Harnleitersteines $\mathbf{2 3 8 3}$

Schlüssel, genetischer 2066

Schock u. Hitzschlag 1696

-, kardiogener u. Therapie $\mathbf{1 7 5 2}$

- u. LDH 1883

- u. Myokardinfarkt 1640

- u. Thrombolyse 2293

Schoeller-Junkmann-Preis 1970 1549

Schrittmacher bei Achalasie mit Herzstillstand 2192

- u. Elektrochirurgie 1469

Schrumpfnieren u. Plasma-Renin 2264

Schule u. Circuit, Training 2126

Schulerfolg u. Geschwisterzahl 1629

Schwabe-Preise 1969 u. 1970 2011

Schwachsinn u. Chromosomen 2676

-, klinische Pathologie 1774:

-, Vererbung 2519

Schwangerschaft u. Anti- $\mathrm{Rh}_{0}(\mathrm{D})$ Prophylaxe 1433

- u. Bakteriurie 1553

-, Geschlechtsdiagnose 2663

-, Risikofälle $2253^{*}$

- u. Röntgenreihenuntersuchung 2127

Schweigepflicht, Bruch 1504 R $1768 \mathrm{R}$

- u. Krankheitsmeldung $1767 \mathrm{R}$

- u. Unterlagenherausgabe

- u. Vertrauensarzt-Angestellte $1423 \mathrm{R}$

Schweißtest u. Mucoviscidose 1677

Schwerhörigkeit, Merkblatt 2067

- u. Nephropathie 2642

Schwestern, Kinderheilkunde 2573*

Schwindel u. Hydergin 2431

Sedimentationsanalyse bei Paraproteinurien 2558

Sehnerv u. Ovulationshemmer 1762

Sekretin u. Pankreas-Angiographie 1970

u. Serumanalyse 2086

Sekretionsanalyse nach Kay u. Endoradiosonde 2274

Sekretolytika bei Bronchitis $\mathbf{2 2 3 4}$

Sembrinna u. Blutdruck 2083

Seminom u. Cyclophosphamid 2588

Sengstaken-Blakemore-Sonde bei Ósophagusvarizen 1477

Sepsis, Lungenbefunde $\mathbf{2 2 2 6}$

- u. Staphylokokken, Lysismuster u. Antibiogramm 1451

- durch Vibrio fetus $\mathbf{2 4 8 4}$

Septikämie, Vibrio fetus 2400

Serotonin u. Parkinson 1942

- zur PhonokardiogrammDiagnostik 1751

Serpasil u. Blutdruck 2083

Serumanalyse u. Pankreasstimulation 2086

Serumcholesterin, erniedrigtes 1546 
Shunt, atrioventrikulärer bei Lungengeschwülsten $\mathbf{1 4 1 9}$

- portocavaler u. Octapressin 1679

Shuntoperationen bei Osophagusvarizen 1479

Silikonausguß zur Dickdarmkrebs-Diagnose 2186

Silikose 2290

Simian-Viren $1843^{*}$

Simon, G. u. Nephrektomie 2247

Simon-Medaille 2362

Sinnesphysiologie bei Amputierten 2371

Sinustachykardie, Therapie $\mathbf{2 6 7 0}$

Skelet, kindliches u. Mineralsalzbestimmung 1983

- Röntgendiagnostik 2183*

Skeletfehlbildungen u. Blutverlust der Mutter 2342

Sekeletmuskel u. LDH $\mathbf{1 8 8 2}$

Skeletveränderungen u. Hyperparathyreoidismus 2596

Skeletszintigraphie 2704*

Soor u. Borsäure 1841

Sorbit bei Diabetes 2659

Spasmo-Analyse des Harnsteinleiden $\mathbf{2 3 8 1}$

Spiegelkurs in HNO $1897^{*}$

Spironolacton bei Hyperaldosteronismus 2052

Splenoportographie, Röntgenverbesserungen $\mathbf{1 4 8 5}$

Spondylitis ankylopoetica $1586^{*}$

Spontanpneumothorax 2408

Sport u. Circuit-Training 2126

Sprue u. Alkalose 1446

Stadtbevölkerung u. Arteriosklerose 2575

Staphylokinase 2352

Staphylokoagulase 2351

Staphylokokken, Metastasen der Lunge 2227

-, pathogene u. Aktivität 2349

- u. Penicilline 2608

Staub- u. Lunge $2460^{*}$

Staubeinwirkung auf Lungen 2439

Staublunge des Bergmannes 2378

Stauungsleber u. $\gamma$-GT-Aktivität 1911

Stauungszirrhose u. Magenresektion 2169

Steatorrhoe ú. Dünndarmdivertikulose 2101

- u. Ig A-Mangel 2027

Sterbefälle u. Eheschließungen 1845

- 1968, Zunahme 1777

Sterilisation 1427 *

Steriven zu Infusionen $\mathbf{1 7 9 4}$

Steroidakne 1395

Steroide bei Hitzschlag 1696

Steuerrecht fuir Auslandsreisen $1704 \mathrm{R}$

Stickoxidulmethode u. zerebrale Insuffizienz 2654

Still-Syndrom, Azathioprin u. Knochenmark 2268

Stoffwechsel der Blutkörperchen 2360"

Stouffer-Preis 19692463

Strafrecht des Arztes 1843*

Straftaten, Kinder als Opfer 1899

Strahlenbehandlung des

Bronchialkarzinoms 1593

Strahlenbiologie der Tumorbehandlung $2573^{*}$

Strahlenfibrose $\mathbf{2 2 9 0}$

Strahlengenetik $2183^{*}$
Strahlenpathologie, klinische $1427^{*}$

Strahlentherapie, Echinacintest zur Leukozytenprovokation 1406

Straßenverkehrsunfälle I/1969 2362

-, Zahlen in Europa 2529

Streptokinase bei Herzinfarkten 2296

- u. Thrombolyse 2293

Streptokokken, Metastasen der Lunge 2227

Striatum u. Parkinson 1942

Stromzeitvolumina, Registrierung 1621

Strophantin bei Herzschock $\mathbf{1 7 5 3}$

Struktur u. Funktion 2129*

Struma u. Testosteron 2527"

Strumen, blande u. Novothyral 2480

Studenten u. Psyche $2252^{*}$

Studienmöglichkeiten in Ulm 1469

Studierende, Zahl 2067

Stuhl, Lysozymausscheidung 1459

Subaortenstenose, muskuläre 2579

Succus liquiritiae u. Magenulcus 2567

Suchtgefahr bei Distraneurin 2299

Sudeck-Syndrom, Therapie 1505

Sulfomucopolysacharide, Stoff wechsel $\mathbf{1 8 7 8}$

Sulfonamide bei Pyelitis 1939

Supratentorielle Eingriffe $2410^{*}$

$\mathrm{SV}_{40}$-Virus u. Impfstoffe $\mathbf{1 6 6 3}$

Symmetrel bei Grippe 2511

Sympathikolytika, Neuroplegika 1941

Syndrom, bronchitisches $1467^{*}$

Syndrome, zervikale $2527^{*}$

Syphilis, Neurolues u. Therapie 1486

Szintigraphie der Leher $\mathbf{1 5 7 5}$, 2003

\section{$\mathrm{T}$}

Tabakrauchen, Pharmakologie 1951*

Tachykardie, paroxysmale u. Therapie $\mathbf{2 6 7 0}$

-, supraventrikuläre,

paroxysmale $2574 *$

Tannhauser-Preis 2254

Taractan, Wirkung 1943

Taschenbuchprogramm 2663

$99 \mathrm{mTCS}$-Kolloid bei Leberszintigraphie 1574

Technik der Infusionsbehandlung 1793

Telemetrie, biomedizinische Anwendung 2649

Terbolan, Wurzelreiz? 2408

Testosteron u. Struma $2527^{*}$

Teststreifen auf Glucose, Fehler 1566

Tetanusauffrischimpfung 1707

Tetanusimpfung u. Herzinfarkt 1895

Tetrachlorkohlenstoffvergiftung,

Klinik u. Therapie $\mathbf{1 6 4 6}$

Tetracyclin u. Nocardiose 2507

- bei Pyelitis 1939

Therapie, aktuelle 2008*

-, Manual 1468*

-, physikalische 2008
Thermographie $1586^{*}$

Thiamin u. megaloblastische Anämie $\mathbf{2 0 0 0}$

Thoraktomie u. Bronchialkarzinom 1561

Thoraxdiagnostik, röntgenologische $2621^{*}$

Thorium X bei $\mathrm{M}$. Bechterew 2459

Thrombin, Sequenz 2696

Thromboembolie, arterielle 2315

Thrombolysetherapie $\mathbf{2 2 9 2}$

Thrombopenie, Immunsuppressiva $\mathbf{2 4 4 7}$

Thrombose u. experimentelle Arteriosklerose 2662

Thrombosen am Auge u. Ovulationshemmer $\mathbf{1 7 6 4}$

- u. Thrombozyten 1953"

Thrombotest u. Quick-Test 2525

Thrombozyten u. Erythrozyten $2360 *$

-, Funktionsprüfungen 1735

- u. Hämostase 2527"

- u. Imurel 2270

Thrombozytenfunktion $1953^{*}$

Thrombozytopenie $u$. Retraktion $\mathbf{1 7 3 8}$

Thymektomie u. Allergie 2062

Thymuskarzinom, Polyneuropathie $\mathbf{2 1 0 2}$

Thyreotoxikose u. Peritonealdialyse 2615

Thyroxin u. Hyperthyreose $\mathbf{2 3 8 6}$

- u. Trijodthyronin $\mathbf{2 4 7 8}$

Tiefenpsychologie des Phantomschmerzes 2371

Tier u. Viren in Impfstoffen $\mathbf{1 6 5 8}$

Tierbisse u. Pasteurella multocida $\mathbf{1 8 1 6}$

Ticrexperimente, Unsitte 2453

Tierquälerei, Preise 1777

Tiffenautest u. Asthma 1803

Todesfeststellung 2405

Todeszeitbestimmung 2183*

Tolbutamid zu Leukose? 1625

Tollwutschutzimpfung von Hunden 2620

Tollwutvirus 1697

Tophus bei Gicht, Röntgendiagnostik 1910

Totalkapazität der Lunge $\mathbf{1 7 8 5}$

Toxine u. Herzbeteiligung $\mathbf{1 7 5 8}$

Toxogoninwirkung, esterolytische 1583

Toxoplasmose, Lymphadenitis 2197

Tracheobronchialaspirat $\mathrm{u}$. Immunglobulinkonzentrationen 2555

Transaminasen u. CholezystoCholangiographie 2020

Transferrin u. PneumokokkenMeningitis 2150

Transfusionsverweigerer $2122 \mathbf{R}$

- u. Arzt 2005 R

Transmissions-Elektronenmikroskopie $\mathbf{1 8 3 4}$

Transplant-Wirt-Reaktion 1845

Transplantate u. Immunsuppression 1422

Transplantation der Leber $\mathbf{2 2 3 9}$

Transplantionsimmunität $1708^{*}$

Transplantationslunge 2202

Trasicor 2560

- u. CSN-Stimulator $\mathbf{1 7 2 0}$

Traumerleben Amputierter 2369

Tretamin, immunsuppressive Therapie $\mathbf{2 4 4 4}$

Triamteren bei Hyperaldosteronismus 2052

Trichomonadeninfektion,

extragenitale 2524

Triglyceride u. Diät 2308

Trijodthyrosin-Test 1455

- u. Thyroxin 2478

2,4,6-Trimorpholinopyrimido(5, 4-d)-pyrimidin u. Plättchenaggregation 1739

Trizymal, Aktivität 1871

Tropenreisen, Ratgeber 2459"

Trunksucht als Krankheit $1624 \mathrm{R}$

Trypsin in Arzneien 1870

-, Sequenz 2696

- u. Tollwutvirus 1701

Tuberkulinprobe, Differentialdiagnostik 2568

Tuberkulose des Gehirns 2281

Tuberkulose $1952^{*}$

-, Lymphadenitis 2196

- u. Mycetome 2047

-, PAS u. Urolithiasis 1491

-, Urolithiasis u. PAS-Calcium 1929

Tuberkulosekranke, Zahl 2462

Tubulopathien, primäre $2704^{*}$

Tumoren u. L-Asparaginase 1669

-, autochthone als Testmodelle 2250

- u. Gerinnungshemmung 2706

- durch Immunsuppressiva 2568

- u. LDH 1884

-, lymphoretikuläre 2201

,-- u. Cyclophosphamid 2583

-, Metastasen in Lymphknoten 2201

Tumorbekämpfung, präventive 2015

Tumorbestrahlung, strahlenbiologische Aspekte 2573"

Tumorentzugshypoglykämie 1783

Tween 80 u. Tollwutvirus 1701

Typhus u. Ampicillin $\mathbf{2 6 1 0}$

- -Schutzimpfung 1425

$\mathrm{U}$

Ulcus u. Carbenoxolon-Natrium 2566

- duodeni u. Betazoltest 2278

- -, Vagotomic 1516

- ventriculi u. Betazoltest 2278

Ulkuschirurgie $\mathbf{1 5 1 3}$ 
Urolithiasis, Therapie 2382

Urologie u. Kryochirurgie 1497

Uterus u. Oxytocin 2613

Uteruskarzinom, Früherkennung 2016

UV-Licht u. Tollwutvirus 1702

V

Vaccination, Frühimpfung 2403

Vademecum psychopharmacologicum 2662

Vagotomie u. Darmmobilität 1513

Valium, Nebenwirkungen 2571

-, Sexualstörungen 1467

Varikose des Ósophagus, Therapie 1476

Vasopressin u. Oxytocin 2611

Vasopressoren u. Hitzschlag 1696

Vena anonyma-Punktion 1797

- cava inferior-Katheder 1796

- saphena magna zur Venae sectio $\mathbf{1 7 9 5}$

- subclavia-Katheder 2682

Venae sectio bei Infusionen $\mathbf{1 7 9 5}$

Venendruck u. Octapressin $\mathbf{1 6 7 9}$

- u. Quinton Shunt 1604

Venenerkrankungen 2009*

Venenthrombosen, Therapie 2292

Verdauungssystem, Chirurgie $2064^{*}$

Vergiftung u. Herzmuskelnekrosen 1852

- mit Tetrachlorkohlenstoff $\mathbf{1 6 4 8}$

Veritol u. PhonokardiogrammDiagnostik 1752

Verkehrsunfälle, Hilfe 1549

Vernon-Stouffer-Preis 2463

von Verschuer, Otmar Freiherr 2407

Versuche am Menschen 2528*

Vertrauensarzt u. Schweigepflicht $1423 \mathrm{R}$

Verwirrtheitszustände, Therapie 2571
Vibrio fetus, Erreger 2399

- -, Sepsis u. Meningitis 2484

Vigantol u. Mineralsalz bestimmung 1986

Vinca rosea, immunsuppressive Therapie $\mathbf{2 4 4 4}$

Vincristin bei Leukämie 1993

Vinebergsche Methode der Arterienimplantation 1904

Viren der Geflügel u. Impfstoffherstellung 1659

- u. Herzentzündung 1758

- in Impfstoffen 1657

- u. RNS-Verabreichung 1629

- u. Nervensystem 2071

Virofral bei Grippe 2511

Virologie 2661

Virus-RNS u. Basensequenz 2254

- der Tollwut 1697

Virushepatitis, LeberkomaTherapie 2235

- u. Australia-Antigen 2294

Virusinfektionen u. Lymphadenitis 2196

- u. Parkinson-Syndrom 2525

Viruskrankheiten u. Rifampicin 1954

Virusvermehrung, Hemmung 1540

Vitalkapazität, Normalwerte 1785

Vitamin B ı bei Anämie 2002

- $B_{12}$ bei Leberleiden 1770

- $\mathrm{B}_{12}$-Malabsorption u. Dünndarmdivertikulose 2101

- B12 u. Methylmalonacidurie 1469

- D u. Fluor bei Osteoporose 1733

- D, Halbwertzeiten 1955

- Dз u. Mineralsalzgehalt 1985

- D in Nahrungsmitteln 1997

- D, Stoßbehandlung 1877

- D, Wirkungsweise 1996, 2302

- K u. Harnsäure 2653

Vogelzüchterlunge $\mathbf{2 6 2 9}$

Vorhofflattern, Therapie 2670

Vorhofflimmern, Therapie 2670

Vorsorgeuntersuchung bei Krebs 1582

\section{W}

Wadenschmerzen 2525

Wasserhaushalt u. Leber $1627^{*}$

Wachstumshormon u. Glucagon 1509

-, postoperativer 1898*

Weber-Preis 1845

Wehen u. Oxytocin 2613

Weißrußland, Fieber 2462

Wieland-Preis 2362

Wilms-Tumor im ersten

Lebensjahr 2598

Wilsonsche Krankheit 1628*

Wirbelsäule u. Beinamputation 1706

-, Fehlbildungen u. Blutersatz 2342

Wiskott-Aldrich-Syndrom 1845

Wörterbuch, englisch-deutsches 2661:

Wolter, H. H. 1464

Würzburg, Augenbank 1588

Wunddehiszenzen, aseptische 140

\section{$\mathrm{X}$}

X-Chromosom u. Anstaltspatienten 2675

Xanthin-Harnsteine nach Allopurinol 1549

Xga-Blutgruppensystem u. Geschlechtsverhältnisse 2066

Xyloseresorption bei Alkoholismus 1532, 1893, 2297

\section{Y}

Yabavirus u. Impfstoffe $\mathbf{1 6 6 3}$

\section{Z}

Zahnextraktion bei Hämophilie 1886
Zahngranulome, CortisonDauertherapie 1467

Zahnpasten, jodhaltige u. Schilddrüse 2408

Zelle u. Gefrieren 1498

Zellschädigung 1548

Zentralvenenthrombose u. Ovulationshemmer $\mathbf{1 7 6 2}$

Zerebralsklerose, Therapie von Verwirrtheitszuständen 2571

Zervixkarzinom, Früherkennung 2016, 2453

Zigarettenraucher u. Myokardinfarkt 2646

Zirrhose u. $\alpha_{1}$-AntitrypsinMangel 1955

-, Faktoren 1828

- u. $\gamma$-GT-Aktivität 1911

- u. Laporoskopie 1633

-, Laparoskopie u. Histologie 2121

- u. Magenresektion 2169

- u. Narbenleber 1637

Zoster, Muskelparesen $\mathbf{1 8 6 1}$

Zoxazolamin u. Harnsäureausscheidung 2653

Zuckeraustauschstoffe bei Diabetes 2659

Zukunftsplanung 2615

Zungentumoren 1668*

Zwerchfellhernien $1844^{*}$

Zwölffingerdarm, Tonus u. Mobilität nach Vagotomie u. Pyloroplastik 1513

Zwölffingerdarmgeschwür u. Betazoltest $\mathbf{2 2 7 8}$

Zyklothymieprophylaxe, Lithium 2513

Zystitis, interstitielle 2620

Zystogramm-Reflux 2056

Zytochemie bei Leukämien 2320

Zytogenetik, Wörterbuch 2129 "

Zytologic u. Histologie 2300"

Zytomegalie, Antikörperuntersuchungen 1966

- bei Nierentransplantation 2202

Zytomegalieviren $2661^{*}$

Zytostatikum, Neues bei Myelom 2498 
A

Adam, W., Ulm 1426

Adolphs, H.-D., Düsseldorf 2634

Affoltcr, H., Basel 1640, 2460*

Ahrens, G. $1775^{*}$

Allert, M.-L. 2460*

Allgöwer, M. 1468"

Althoff, H., Hamburg 2415

Amor, H., Innsbruck 2669

Amrein, R., Basel 1705

Anderson, W. 1708*

Andrassy, K., Heidelberg 1765

Andrews, J. R. 2573*

Anger, G., Erfurt 2495

Angst, J. 2128*

Anschütz, Darmstadt 2704*

Anschïtz, F., Darmstadt 1951

Arnold, Essen $2705^{*}$

Arnold, M. 2253*

Aronow, L. 1952*

Artz, C. P. $2128^{*}$

Asamer, H., Innsbruck 2338

Asante, F., Tübingen 1987

Ask-Upmark, E. 2705:

Atkinson, R. S. $1468^{*}$

Aust, W., Marburg 1688

\section{B}

Bachmann, K., Erlangen 1903, 2191

Bachmann, K. D., Köln-Lindenthal 2598

Baicr, M., Hamburg 2644

Bandmann, H.-J., München 1841 Bartelheimer, Hamburg $1774^{*}$ Barth, Giessen 1427*

Bauditz, W., Hamburg 1604 Bauer, J., Los Angeles,
Calif./USA 1893

Bauer, K. H. 1548*

Baufeld, H., Erfurt 2495

Baum, P., Mainz 2118

Baumann, G., München 2296

Baumgartl, F. 2064*

Beck, K. 2460*

Becker, Bonn 1586*,2461*

Becker, Göttingen 2301*

Becker, H. D., Köln-Lindenthal 2135, 2202

Becker, J., Plettenberg 1543

Becker, P. E. 1951*

Becker, W. 2064*

Beckmann-Wilkes, C. 2307

Beckmann, D. 1773 *

Beckmann, H., Hamburg 2320

Begemann, H. 2661*

Begenat, H., Düsseldorf 1724

Behrend, Trude, Bad Nenndorf 2389

Beickert, A. 1427*

Beintema, D. J. 2128*

Beisenherz, W., Hannover 2616

Benini, A., Zürich 1617

Berde, B. 1774*

Berensmann, R., Stuttgart-

Degerloch 2526

Bergentz, S. E. 1468*
Bergsma, D. 1628*

Beringer, A., Wien 2028

Berndt, H., Berlin-Buch 1559,

1893, 2003, 2181, 2452

Berndt, W., Hamburg 1870

Bernhard, S. A. $2706^{*}$

Beske, F. $1708^{*}$

Beyer, H. 1587"

Bieger, D., Kiel 1506

Bierstedt, P., Hamburg 2241

Biesalski, Mainz 2252*

Binder, H., Homburg/Saar 1972

Biniek, Tübingen $2185^{*}$

Birnmeyer, G. 1897*

Bläker, F., Hamburg 1978

Blankart, R., Luzern 1640

Blasius, Ch., Tübingen 2501

Blunck, Hamburg 2154"

Bock, Tübingen 2128*

Bockelmann, P. 1843*

Bode, Ch., Marburg 2086

Bohle, A., Tübingen 1673

Bollinger, A., Zürich 2347

Bonin, Frankfurt 2662*

Bontemps, M., Kiel 2435

Borm, D., Kiel 1401

Bottke, H. 1468*

Boulle, S. 1508*

Brandt, H., Berlin-Dahlem $1946 \mathrm{R}$

Brauer, W. 2704:

Braun, O. H., Pforzheim 1458

Braun, W., Heidelberg $\mathbf{1 6 8 5}$

Braun-Falco, O., München 1466

Breddin, K. 1953*

Brehm, G., Erlangen 1677

Brehm, H. 1952*

Bressel, M. 2460*

Broicher, H., Grevenbroich 2429

Brothwell, D. 2185*

Bruchhausen, D., Bonn 1651

Brügmann, E., Minden/Westf. 1944

Brühl, W. 2459*

Brüster, H., Düsseldorf 1799,

2539

Brunner, E., Wien 2107

Buckingham, R. A. 2064*

Buckup, H., Bochum 2375

Bücherl, E. S. 1898"

Bühlmann, Zürich 1468*

Bünning, Tübingen 1953*

Bütikofer, E., Bern 2627

Biittner, Kiel 1668*, 1775*

Bundschu, H. D., Tübingen 1888

Bunjes, W. E. 2661"

Burck, H.-Chr., Tübingen 1503

Burger, H., Zürich 1640

Burian, K., Wien 2028

Busch, H., Mainz 2118

\section{C}

Cain, H., Stuttgart 1607, 2173, $2704^{*}$

Calne, R. Y. $1468^{*}$

Campbell, E. J. M. 2410*

Cantarow, A. 2361*

Canzler, H., Hannover 2307
Casarett, G. W. 1427*

Cen, M., Aachen 1970

Choné, B., Heidelberg 1406

Ciompi, L. 2185*

Clauditz, S., Giessen 1615

Coper, H., Berlin 1467

Cotta, H., Heidelberg 1505

Crcutzfeldt, Göttingen 1625, $1668^{*}$

Czembirek, H., Wien, 1574

\section{D}

Dahlmann, W., Göttingen 2682

Dahm, K., Hamburg 1513

Daniels, J. 2301*

Dardenne, M. U., Bonn-Venusberg 1651

David, Erlangen 2065*

Daweke, H., Düsseldorf 2535

Debrunner, F., Basel 1640

Decker, R., Gelsenkirchen-Buer 1553

Delius, L., Bad Oeynhausen 2009*, 2172

Demling, L., Erlangen 1466, 1666, 1771

Denck, H., Wien 2107

Deutsch, E. 2360*

Deyhle, P., Erlangen $\mathbf{2 2 7 4}$

Dicnstl, F., Innsbruck 2669

Dicthelm, L. 2184*

Diethelm, L., Mainz 2593

Dihlmann, W. 1586*

Dihlmann, W., Aachen 1506, 1909

Dischler, W. 2460*

Dittrich, H., Erlangen 1903

Dittrich, P., Innsbruck 2338

Dobrowolski, L., Warszawa 2060

Dönges, K. G., Essen 1825

Döring, München 1897*, 1952*

Doll, E., Freiburg i. Br. 2563

Dombrowski, H., Marburg/Lahn 2155

Doss, M., Marburg/Lahn 2392

Drescher, J., Kiel 2415

Driesen, W., Tübingen 1418

Drischel, H. 1953"

Duck, H.-J., Leipzig 2060

Dudeck, J., Mainz 2473

Dürr, F., Tübingen 1441, 1888

Düx, Bonn 2008*

Dume, Th., Düsseldorf 1646, 2262

Dunker, S., Frankfurt a. M. 2226

\section{E}

Eastham, R. D. 1775*

Eckart, J. P., Berlin 1679

Eckermeier, L., Hamburg 1730

Edel, H. H., München 2082

Eder, M., Köln-Lindenthal 2326

Effert, Aachen 2527*, 2574*

Eggers, Giessen $1843^{*}$

Eggers, H. J., Giessen 1540

v. Egidy, H., Mainz 1917

Egli, F., Basel 2334
Ehrengut, W., Hamburg 2403

Ehringer, H., Wien 2107

Eickenbusch, W., Kiel 2478

Eigler, F. W., Köln-Lindenthal 2202

Eisenburg, J., München $\mathbf{1 8 8 2}$

Elbel, H., Bonn 2259

Engelmeier, Essen 2252*

Engels, A.-M., Essen 1825

Engelsing, B., Wiesbaden 2059

Enlow, D. H. 1898*

Erdmann, W. D. 2455

Esser, G., Bonn 1476, 1713

Esser, I., Saarbruicken 1959

Euchenhofer, M., Stuttgart 1441

Ewerbeck, H. 2253*

Eycr, H., München 2524, 2251

\section{F}

Feige, G., Leipzig $\mathbf{2 1 6 7}$

Felgenhauer, K., Köln-Lindenthal 1583

Felson, B. $2622 \%$

Fernholz, H. J., Aachen 1909

Filippini, L., Luzern 1577, 1619, 2392

Finke, J., Tübingen 2033

Fischer, K., Hamburg 1978

Fischer, K., Tübingen 2076

Fischer, M., Frankfurt 2583

Fischer, M., Wien $\mathbf{2 1 4 5}$

Fischer, R. 2135

Fischer, R., Köln-Lindenthal 2284

Fischer, W., Zürich 2685

Fleischer, Hamburg 1897*

Fliedner, T. M., Ulm 1426

Fourmann, P. 1774 *

Franke, Würzburg 1844*

Franken, F. H., Düsseldorf 1839 , 2007, 2299, 2397

Franz, Ulm 1548*

Frey, E. K. 1668:

Fried, K. H., Berlin 2240

Friedberg, K1. D. 2455

Friedberg, V. 1627*

Friedmann, G., Köln-Lindenthal 2547

Frik, Aachen 2622*, 2704"

Frik, W., Aachen 1970

Frischkorn, Göttingen 1587*

Fritzsche, W., Frankfurt a. M. 1911

Fuchsig, P., Wien 2145

Fuhrmann, Giessen 1951*, 2129", $2183^{*}$

\section{G}

Gabka, J. 2361*

Gärtner, Kiel 2184*

Gardner, L. I. 2184*

Gartmann, J., Arosa 2234

Gattiker, H., Zürich 2579

Gedigk, P., Bonn 1894

Gehrmann, G., Wuppertal-

Barmen 2063, 2182, 2661*

Gellis, S. S. $1507^{*}$ 
Gerb, A., Ulm 2488

Gerlach, E. 2360*

Gerok, W. 2704*

Gerok, W., Freiburg 2593

Gerth, H.-J., Tübingen, 2076, 2510

Gilfrich, H. J., Mainz 2473

Gillich, K. K., Hannover 2616

Gillmann, H., Ludwigshafen $2009^{*}, 2359$

Glaser, E. M. 2065"

Glassner, K., Düsseldorf 1799

Glauner, R. 2704*

Gleiss, J., Oberhausen-Sterkrado 2449

Gleissner, P., Tübingen 1987

Göbel, U., Düsseldorf 2539

Goebell, H., Marburg 2086

Goerz, G., Düsseldorf 2040

Goetz, O., München 1966

Goldstein, A. 1952*

Goschenhofer, D., Hamburg 1410

Gossmann, H. H., Siegen 2155

Gottstein, U., Kiel 2182

Grabensee, B., Düsseldorf 2262

Graciansky, de P. $1588^{*}$

Granz, Leipzig 2460*

Graucob, E., Lübeck 2415

Greef, K., Düsseldorf 2062

Green, M. M. 2129*

Greither, A. $1775^{*}$

Gressner, P., Göttingen 2682

Gries, F. A., Düsseldorf 2307

Grill, P., Grevenbroich 2429

Gröbner, W., München 2652

Grömig, U., Lübeck 1545 R

Groh, H., Köln-Müngersdorf 1626

Gropp, Freiburg 1508*

Gross, D. 1952", 2185*

Gross, R., Köln-Lindenthal $\mathbf{1 7 3 5 ,}$ 2326

Grossmann, H. D., Heidelberg 2033

Grote, W., Düsseldorf 2342

Gruber, U. F. $1468^{*}$

Grün, Düsseldorf $1427^{*}$

Grumbach, A. 25 7 *

Gruss, J. D., Heidelberg 2315

Gsell, O. 1774*

Gütgemann, A., Bonn-Venusberg 1476, 1713

Günther, Frankfurt 1508*

Günther, D., Düsseldorf 1593

Günther, O., Frankfurt a. M. 1421, 2355

Günther, R., Innsbruck 2338

Günther, W. 1468*

Gürich, St. Andreasberg 1952"

Gütgemann, Bonn 1844:

Gutschmidt, A., Zürich 1640

\section{H}

Haase, G. $1548^{*}$

Habib, R. 2621*

Habighorst, L. V., Mainz 1917

Haemmerli, U. P., Zürich 2063

Hänze, S., Manz 2360

Haering, M., Düsseldorf 1433

Häring, R., Berlin 1679

Hafter, E., Zürich 1495

Hagen, E. $2527^{*}$

Hagen, W. 2301*

Hager, W., Essen 2160

Haike, H. J. 2459

Hall, München 1665

von Haller, E. $2460^{*}$
Haller, J. 1897*

Hammersen, F. D. $1952^{*}$

Hansen, H. G., Lübeck 2415

Hanshaw, J. B. 2662*

Harbauer, Frankfurt 1775

Harbers, E., Lübeck 1948, 2129", $2410^{*}, 2706^{*}$

Hardy, J. D. $2128^{*}$

Harper, H. A. 1586"

Harrwich, G., Erlangen 1703

Hartmann, K. 1547

Hartwich, G., Erlangen 1506

Haschem, J., Bonn 1651

Hasselblatt, A. 2455

Haupt, E., Kiel 2478

Hauschild, G., Leipzig 1491, 1928

Havemann, K., Marburg/Lahn 1867

Haynal, I. $2574^{*}$

Heberer, Köln 1952*

Heerklotz, B., Erlangen 1473

Hegemann, G., Erlangen 1903

Hegglin, R. 2661*

Hehrlein, F. W., Gießen 1522

Heidland, A., Würzburg 1568

Heilmann, K., München 2289

Heinecker, R., Kassel 1626, 1841

Heinrich, K., Mainz 2528*

Heintz, Aachen 2252"

Heintz, R. 1843*

Heister, R. 1468*

Heitmann, R., Köln-Lindenthal 1583

Helm, E., Frankfurt 1816

Helm, E. B., Frankfurt a. M. 2484

Helms, M. 2460*

Henneberg, U., Berlin 1679

Hennekeuser, H.-H.,

Freiburg i. Br. 2284, 2593

Hennemann, H., Würzburg 1568

Henning, H. V., Göttingen 1873

Henschler, D., Würzburg 2126

Herms, W. 2262

Herms, W., Düsseldorf 1646

Herrmann, A. 1586"

Hermann, J., Hannover 2616

Herrlinger, R. $2253^{*}$

Herrmann, J., Hannover 2386

Herter, Bonn 1842

Hertl, Mönchengladbach $1708^{*}$, $1898^{*}, 2461^{*}$

Hertl, M. 2573*

Hertz, C. W. $1843^{\text {* }}$

Herzberg, Bremen 1508*

Herzog, H., Basel 1785

Hess, W., Schömberg 2569

Hesse, P., Erfurt 2495

Hessing, J., Düsseldorf 2675

Hilbert, L., Frankfurt a. M. 2583

Hilgenberg, F., Münster 1877

Hilscher, W., Düsseldorf 2637

Himmelbach, E., Düsseldorf 1799

Hirsch, H. A., Frankfurt a. M. 1553

Hobby, G. L. 2065*

Hochheuser, W., Augsburg 2488

Hodler, J., Bern 1417

Hohenfellner, R., Mainz 1626 , $2460 *$

Holinger, P. H. 2064:

Holle, F. 1507"

Hollingsworth, J. W. 2065*

Hollmann, W., Köln-Müngersdorf 2127

Hollwich, F., Münster 1761

Holzmann, M., Zürich 2182

Horànyi, I. 2185:

Hornbostel, H. 2008*

Hornstein, O. $1775^{*}$

Horst, W., Zürich 2685

Horster, F. A., Düsseldorf 2409
Hoyle, L. 2662*

Hraba, T. 1508

Huber, F. B., Zürich 1937

Hübner, G., Köln-Lindenthal 2202

Hüls, W., Zürich 2685

Hüter, K. A. 2253"

Hüther, W., Münster 1877

Huffmann, G., Köln-Lindenthal 2299

Hull, R. N. 1843*

I

Immich, H. 1844*

Ippen, H., Düsseldorf $1427^{*}$, 1841, 2009*

Iravani, J., Bochum 1586

Irmscher, K., Düsseldorf 2634

Irnich, W., Aachen 2649

Irsigler, K., Wien 1532, 2297

Jacobi, H., Freiburg i. Br. 2550

Jahnke, K., Wuppertal-Elberfeld 2307, 2335

Jakob, Zürich $2301^{\prime \prime}$

Jancar, J. 1775*

Janz, Ilten/Hannover 1951*

Janz, D. 2527"

Janzen, R., Hamburg 2408 .

Jeljaszewicz, J., Warschau 2349

Ientschura, Mannheim 2361"

Jester, H. G., Hannover 2616

Jörgensen, Göttingen 2410*

Joist, J. H., Köln-Lindenthal 2135

Joos, H., Basel 1785

Jucker, E. 1427"

Judmaier, G., Innsburck 2669

Jülich, Stuttgart 2461"

Jung, K., Ludwigshafen 1654 , 1655

Junge-Hülsing, G., Münster 1877

Jurna, I., Homburg/Saar 1941

\section{$\mathrm{K}$}

Kabarity, A., Düsseldorf 2675

Kähler, H. J. 2528*

Käser, O. 1627"

Käufer, C. $2183^{*}$

Käufer, C., Bonn-Venusberg 1713

Kagan, B. M. 1507

Kahlke, W., Heidelberg 1547

Kaiser, Augsburg 1427" 2008*

Kaiser, H., Augsburg 1467, 1772, 2232

Kaiser, N., Kiel 1401

Kaiser, R., München 2467

Kalff, G., Aachen 1970

Kalman, S. M. 1952*

Kaltwasser, P., Frankfurt a. M. 1518

Kanter, H. 2461*

Kaplan, A. S. $2662^{*}$

Karlson, Marburg 1586", 2361*

Katz, G. P. F., Great Neck, N. Y. 2701

Kaufffmann, E. 1628

Kaufmann, W. 2008"

Kaufmann, W., Tübingen 1441

Kazner, E. 2573*

Keller, J., Dresden 2247

Keller, R., Basel 1785

Kempe, L. G. 2410"

Kepp, R., Gießen 2408

Kerkhoven, P., Bern 1695

Kern, Lörrach $1548^{*}, 1468^{*}$

Keul, J., Freiburg i. Br. 2563

Keuth, U., Neunkirchen-Kohlhof/ Saar 1959

Kiani, B. 2460"

Kirchhoff, Göttingen $1627^{*}$

Kistler, H. J., Zürich 2140

Klaiber, R., Zürich 2678

Klapetek, J., Olmütz/CSSR 2123

Klein, E. 2528*

Klein, H. O., Köln-Lindenthal 2326

Klcinsorge, H. 2009"

Klemm, D., Karlsruhe 2558

Klempt, H.-W., Bad Salzuflen 2345

Klepzig, H. 1628*, 2704*

Klingler, Basel $2573^{*}$

Kliitsch, K., Würzburg 1568

Kluge, R., Hannover 2616

Kluthe, R. 2252*

Kluthe, R., Freiburg i. Br. 1673

Knight, J. 1548*

Knolle, J., Mainz 2118

Knoth, Stuttgart $1773^{*}$

Knüpling, H., Bonn 2259

Koch, F., Heidelberg-Schlierbach 1707

Koch, W., Hamburg 2278

Köhle, Ulm $1773^{*}$

Kölle, G., Garmisch-Parten-

kirchen 2268, 2568

Kohlhaas, M., Karlsruhe-Durlach

$1423 \mathrm{R}, 1464 \mathrm{R}, 1504 \mathrm{R}$,

$1625 R, 1767 R, 1768 R, 1643^{*}$,

$1839 \mathrm{R}, 2005 \mathrm{R}, 2061 \mathrm{R}$,

$2122 \mathrm{R}, 2242 \mathrm{R}, 2243 \mathrm{R}$

$2298 \mathrm{R}, 2350 \mathrm{R}, 2523 \mathrm{R}$, $2570 \mathrm{R}, 2656 \mathrm{R}$

Kolb, H. P., Heidelberg, 1856

Kolb, P. J., Heidelberg, 1856

Kommerell, B., Heidelberg 2235

Konzett, Innsbruck 1774*

Korting, G. W. 2064*

Krämer, D., Homburg/Saar 1972

Krämer-Günther, H., Frankenthal $2617 R$

Kramer, H., Heidelberg 1483

Kramer, K., Erlangen 1983

Kramer, P., Göttingen 1873

Kraus, B., Stuttgart 2173

Krauss, J., Stuttgart 1820

Kraut, H. 1668*

Krayenbuehl, H. 2361*, 2622*

Kremer, Essen 1953"

Kremer, K. 2064*

Krempien, B., Heidelberg 1765 
Kuhlencordt, F., Hamburg 1730

Kukuth, W. 2572

Kunst, H., Köln-Lindenthal 2326

Kuschinsky, G., Mainz 2007, 2571

Kuwert, E., Essen 1697

L

Lahrtz, Hg., Kiel 2478

Landbeck, G., Hamburg 2320

Lang, H. D., Tübingen 2033

Lang, K., Mainz 2118

Lange, Gummersbach $1628^{*}$

Lange, H., Marburg 2155

Lange, M. 2705*

Langen, D. 1708*, 2409*

Langen, P. 2065*

Largiadèr, Zürich 1708*

Laubach, K., Heidelberg 2315

Laubenthal, F. 2523

Lauter, H., Göttingen 2512, 2659

Lavender, R., Gines, Ill./USA 2082

Lechler, E., Köln-Lindenthal 2326

Lechner, K., Wien 2145

Lederer, F. L. 2064:

I.ee, J. A. 1468"

Lee, T. S., Bonn-Venusberg 1713

Lehmkuhl, H. 2301*

Leinweber, B., Giessen 1615

L.eischik, W., Köln-Lindenthal 2505

Lejeune, F. 2661*

Lemberg, G., Marburg 2086

van der Lende, R. 2129*

Lennert, K., Kiel 2194

Leonhardt, H. 2300

Leppla, W. 1898

Leybold, K., Kiel 2478

Leydhecker, W. 1898*

Lichtlen, P., Zürich 1640

Linnewch, Marburg 2621*

Loose, K. E. 2008

Lozano-Tonkin, C., Hamburg 1730

Lübbers, D.-W. 1707"

Lübbers, P., Lübeck-Trave-

münde 1922

Liiders, D. 2461"

Lüllmann, Kiel 1952*

Luft, U. C. $1707^{\circ}$

Lutz, Mannheim 2410"

Lutzeyer, W., Aachen 1666

Lydtin, H., München 2560

\section{M}

Maas, B., Hamburg 1410, 2320

Mainzer, S., Mainz 2284

Majer, M., Bern 1657

Mancke, Hannover 2301*

Manidakis, G., Heidelberg 1406

Mann, Marburg 2253*

Markoff, N., Chur 1829

Martin, H., Frankfurt a. M 1584,1891

Martini, G. A., Marburg/Lahn 2206

Marx, Stuttgart 1843*

Marx, H. H., Stuttgart 2408

Maspes, P. E. 2361", 2622"

Massermann, J. H. $1668^{*}$

Maspes, P. E. 2361*

Mast, H., Münster 2602

Mathieu, H. 2621"

Matsch, J. 2574*

Matussek, München 2460*

Matz, K., Hamburg 1448
Mau, H., Tübingen 1950

Maurer, München 2361*

May, P., Homburg/Saar 2115

Mayer, Mainz 2300*

Mayor, G., Zürich $\mathbf{2 1 4 0}$

McEwen, C. $2009^{*}$

McKusick, V. A. 2410*

McNicol, M. W. 2410*

Meade, R. H. 2662*

Meesmann, W., Assen 2359

Mehnert, H., München 1706, 1950, 2659

Meier-Ruge, W., Basel 2334

Meisner, H., München 1621

Mendel, D. 2527*

Menden, E., Gießen 2620

Menz, H.-P., Tübingen 2501

Mertz, P., Freiburg 1896

Messmer, K., München 1621

Meurer, K. A., Tübingen 1441

Meyer, Göttingen 1668*

Meyer, H. A., Zürich 1640

Meyer, J., Aachen $\mathbf{1 7 5 5}$

Meyer-Beeck, D., Braunschweig 1993

Meyer-Burg, J., Berlin 2018, 2421

Meyer, J.-E., Göttingen $2118^{*}$

Meyer zu Schwabedissen, O.

Achern/Baden 1544

Michaelis, A. 2129:

Miederer, S. E., Erlangen 2274

Mielke, F., Berlin 2102

Miese, A., Haam, Rheinl. 2637

Mills, J. 1668*

Miss, H. D., Wuppertal-Barmen 2307

Missmahl, H. P., Hamburg 2691

Mittelmeier, Homburg 2009*

Möbius, W., Köln-Lindenthal 2281

Möller, U., Marburg 1688

Mörl, H., Halle/Saale 2167

v. Monakow $1547^{*}$

Montz, R., Hamburg 1503

Moormann, A., Würzburg 1568

Moormann, J. G., Homburg/

Saar 2053

Moos, C. 2129*

Morawetz, F., Wien 2605

Moser, K. 2360

Mountcastle, V. B. 1548"

Mühe, E., Erlangen $1 \mathbf{7 8 1}$

Müller Ch. $2185^{*}$

Müller, D., Tübingen 2033

Müller, H. E., Bonn-Venusberg 2149

Müller, N., Karlsruhe 2526

Müller, W. A., Bern 1695

Müller-Bardoff, M., Ulm 2488

Müller-Duysing, W., Zürich 2685

Müller-Plathe, Hamburg 1898"

Müller-Wieland, K., Hamburg 1870

Müntefering, H., Düsseldorf 2637

Mürtz, R., Düsseldorf 1724

Mumenthaler, M., Bern 1585

Mund, J., Freiburg i. Br. 2550

Muntean, E. 2704*

$\mathbf{N}$

Nager, F., Zürich 1640

Naumann, Düsseldorf $1774^{*}$, 2065

Naumann, H. H., Berlin $2183^{*}$

Neth, R., Hamburg 1410, 2320

Neu, O., Frankfurt/Main $\mathbf{1 8 1 3}$

Neubauer, F. W., Berlin 2421
Neuhäuser, G., Erlangen 2519

Neumann, G. 1772

Neumann, G., Stuttgart 1581, 2127

Niederhoff, A., Freiburg i. Br. 1437

Niederhoff, H., Freiburg i. Br. 2000

Niemczyk, H., Mainz 2118

Niemeyer, G., Stuttgart 1735

Niemeyer, W. $2252^{*}$

Nieschlag, E., Mainz 2473

Nissen, Basel 2064", 2128"

Nissen, P., Hannover 2616

Nissen, R. 1844"

Noder, W., Bad Salzuflen 2345

$\mathrm{O}$

Ober, K. G. 1627"

Obes, A. R., Freiburg 2453

Obrecht, P., Freiburg 2571

Ochs, U., Tübingen 2076

Oehme, J., Braunschweig 1939, 1993

Oeser, Berlin 2573*

Oeser, H., Berlin 2015, 2181, 2453

Olbing, H., Essen 1566, 1825

Olsson, O. 2184

Opelt, B., Homburg/Saar 1497, 2053

Ortmans, H., Kassel 2215

Oshima, H., Berlin 2440

Ott, Bad Nauheim-Gießen 1586"

Otte, H.-J. 2184*

Ottenjann, R., Erlangen 2191, 2566

Overzier, C., Mainz 2473

$\mathbf{P}$

Pabst, K., Freiburg i. Br. 1752, 2593

Pakzad, M., Berlin-Neukölln 1563

Pappworth, M. H. 2528*

Paquet, K. J., Bonn-Venusberg 1713

Paschke, R., Emskirchen $2062 R$

Peller, P., München 1966

Pelzl, H., Larburg/Lahn 2690

Penin, H. 2183"

Peters, München 1548*

Petrilowitsch, N. 1953*

Pepys, J. 2460*

Perret, E., Zürich 2678

Pfab, R., Marburg/Lahn 2155

Pfeiffer, E. 2301"

Pfeiffer, K. M. 1844*

Pfister, Karlsruhe 1508*

Pfitzner, W., Düsseldorf 2539

Pia, Gießen $2410^{*}$

Pia, H. W. 1773*

Pichlmaier, H., München 2082

Pilgerstorfer, H. W., Wien 2145

Platt, D., Gießen 1414, 1420

Platt, M., Gießen 1414

Poch'e, R., Bielefeld 1851

Plowright, W. 2662:

Poeck, K., Aachen 2367, 2703

Pöldinger, Basel 2185

Pointner, H., Wien 1532, 2297

Prechtl, H. F. R. $2128^{*}$

Preiss, H., Düsseldorf 2307

Preston, R. L. 2009*

Preter, B., Zürich 2395, 2579

Pribilla, W. 1473, 2356

Prill, A., Göttingen 1743, 2682

Pürckhauer, F. 2301*

Püschel, E., Bochum 2245

Pulverer, G., Köln-Lindenthal 2349, 2572*

Q

Quandt, J., Bernburg/Saale-DDR 1486

Quellhorst, E., Göttingen 1398, 1873

Quirin, H., Freiburg i. Br. 2112

$\mathbf{R}$

Raab, W. 2009*

Rach, K., Berlin 2015, 2453

Radl, H., Wien 1599

Raithel, D., Erlangen 1781

Ramseier, H. 1708*

Rauch, Renate, Kiel 2611

Rautenburg, H. W., Gießen 1750

Rave, O., Münster 1877

Reber, H., Basel 2607

Redenbacher, M., Stuttgart 1820

Rehner, M., Hamburg 2121, 2278

Reichel, G., Bochum 2375, 2703

Reichmuth, J., Luzern 1640

Reifferscheid, Aachen 1507*, $1708^{*}$

Reil, B., Münster 2602

Reinhardt, K., Völklingen 2045

Reiss, K. H., Erlangen 1983

Reploh, H. 2184*

Reploh, H., Münster 1667

Reubi, Bern 1843*

de Reuck, A. V.S. $1548^{*}$

Reumuth, H., Karlsruhe-Waldstadt $\mathbf{1 8 3 2}$

Reuter, Köln 1735, 1953*, 2360*, 2527

Reutter, F., St. Gallen 1640

Rhomberg, F., Zürich 1457

Richter, E., Gießen 1615

Richter, H. E. $1773^{*}$

Riech, P.-Chr., Düsseldorf 1799, 2539

Riecken, E. O., Marburg/Lahn 2206

Riederer, J., Trier 2181

Rieger, H.-J., Gießen 1840 R,

$1947 \mathrm{R}, 2657 \mathrm{R}$

Rieger, R., 2129*

Ringler, W., Aachen 2539

Ringler, W., Düsseldorf 1799 , 1802

Ringwald, E., Bern 1945

Ritz, E., Heidelberg 1765

Ritz, R., Basel 1640

Riva, Bern 1898*

Rodenhäuser, J. H., Marburg/ Lahn 2389

Röhrborn, G. 2183*

Rösch, W., Erlangen 2191

Rösli, R., Zürich 1640

Rössler, H. 1468*

Rosenbusch, G., Aachen 1970 
Sabuncu, N., Göttingen 2682

Sachsse, B., Hösel 2535

Sachsse, R., Hösel 2535

Sachtleben, P., Homburg/Saar 1972, 2573*

Salfeld, Minden 1775*

Saling, E., Berlin 1563

Salzer, Mechthild, Wien 2145

Samman, P. D. 1508*

Sander, J., Tübingen 2501

Sandison, A. T. 2185

Sandritter, Freiburg 2253*

Sapounov, S., Hamburg 1513

Sarre, H., Freiburg i. Br. 1673

Sauer, H., Bad Oeynhausen 1771

Sauer, R. 2307

Schaal, K. P., Köln-Lindenthal 2505

Schade, H., Düsseldorf 2407, 2675

Schadewaldt, H., Düsseldorf $\mathbf{1 7 6 9}$

Schaede, A., Bonn-Venusberg 1717, 1895, 2571

Schäfer, K. H., Hamburg 1410

Schaltenbrand, G. 1897*

Schassan, H.-H., Hamburg 1448

Scheid, Göttingen 1548*

Scheid, Köln 1547*, 1897

Scheiffarth, F., Erlangen $1427^{*}$,

Scheitlin, W., Zürich 2140

Scheler, F. 1898*

Scheler, F., Göttingen 1393, 1873

Schellorg, G., Münster 2602

Schepartz, B. 2361"

Schettler, G. 2128*

Schettler, G., Heidelberg 1464

Schewior, Roland, R., Kiel 2435

Schick, Aachen 1628*

Schiefer, W. 2573"

Schievelbein, H. 1951*

Schimpf, Kl., Heidelberg 2292

Schleiermacher, E. 2183"

Schlegel, K. F., Essen 2359

Schleyer, F. 1548*

Schliack, H., Berlin 1861

Schlierf, G., Heidelberg 1667

Schliesser, Th., München 2621

Schmähl, D., Heidelberg 2249

Schmidt, F. W., Hannover 2251

Schmidt, G. 2457

Schmidt, O. P. $1468^{*}$

Schmidtke, Helga, Hamburg 2320

Schmutzler, R., Giessen 1424

Schneeweiss, U. 1628"

Schneider, C., Hamburg 2631

Schneider, H., Berlin 1861, 2404

Schneider, J., Freiburg i. Br. 1437

Schnyder, U. W., Heidelberg 1990

Schober, H., München 1545

Schölmerich, Mainz 2661*

Schönbeck, M., Zürich 2685

Schöne, D., Leipzig 1491, 1928

Schoenmackers, Aachen $2622 *$

Schoop, Engelskirchen 2009*

Schoop, H. D., Tübingen 2076

Schramm, G. ,Zollikerberg/

Schweiz 1945

Schreiber, H. W. 2064*

Schreyer, H. $2704^{*}$

Schricker, K. T., Erlangen $\mathbf{1 7 8 1}$

Schriefers, K.-H., Bonn-Venusberg 1713

Schröder, E. 2301

Schröder, E., Düsseldorf 1646 , 2262

Schröder, J. 1844*

Schroeder, T. 2183*

Schubert, G. E., Tübingen 1967
Schubert, Helga, Frankfurt a. M. 1813

Schubert, J. C. F., Frankfurt a. M. Stempel, G., Bochum 2375 1813,2583

Schuchardt, K. 1668*

Schuermann, H. $+1775^{*}$

Schuh, F., Kiel 1897, 2008

Schuhmann, R., Ulm/Donau 2229

Schulte, H. D., Düsseldorf 1793

Schulte, Tübingen $1708^{*}$

Schulte, W., Tübingen 2409*

Schultze, Bremerhaven 2621

Schumacher, K., Köln-Lindenthal 2326

Schuster, H.-P., Mainz 2118

Schuster, J., Viernheim 2334

Schuster, W., Erlangen 1983

Schwartz, W., Homburg/Saar 1972

Schweizer, W., Basel 2646

Schwerd, Würzburg 1548*

Schwingshackl, H., Innsbruck 2669

Scriba, Marianne, Wien 2294

Scriba, P. C., München 1455

Seeliger, Würzburg 2461"

Seifert, Hamburg 2185"

Selbach, Berlin $2527^{*}$

Selmair, H., Kassel 2215, 2220

Senning, A., Zürich 2579

Seybold, D., Marburg/Lahn 2155

Shackelford, R. T. 1953*

Shikano, S. 1587*

Shimizu, K. 1587*

Shulmann, S. 2129*

Sickinger, K. 246 $3^{*}$

Sickinger, K., Waldshut 1546

Siebert, H. G., Köln-Lindenthal 2202

Siegenthaler, W. 2008*

Siegenthaler, W., Bonn 2049 2051, 2685

Siegfried, J. 1426*

Sicgfricd, J., Zürich $\mathbf{2 6 7 8}$

Sigg, K. 2009*

Sill, V., Hamburg 1604

Simon, C., Kiel $\mathbf{2 4 3 5}$

Sitzmann, F. C., ErlangenNürnberg 2071

Snell, F. M. 2129*

Sökeland, J. 2460*

Sökeland, J., Homburg/Saar $1497,2053,2115,2620$

del Solar, E., Göttingen 1996

Solbach, H. G., Düsseldorf 2634

Soltermann, W., Thun, Schweiz 2121

Sommer, H., Berburg/Saale-DDR 1486

Sorkin, E. 2662

Spann, Freiburg i. Br. 2183*

Spencer, R. P. 2129*

Spielmann, W., Frankfurt a. M. 2250

Spiess, H. 1585

Spiess, H., München 1707

Spiessl, Basel, 1668

Spitz, H. B. 2622

Sponheimer, K., Fürth/Bayern 2006

Spotnitz, H. 2460*

Stachow, P., Freiburg i. Br. 1437

Stadelmann, O., Erlangen 2274

Stammler, A., Köln-Lindenthal 1529

Staemmler, H.-I. 2300*, 2621*

Starlinger, P., Köln 2406

Steigleder, G. K., Köln-Lindenthal 1393

Steinbereithner, K., Wien 2145

Steinbrunn, W., Zürich $\mathbf{1 6 4 0}$
Steiner, B., Tübingen 1441

Stephan, G., Hamburg 2631

Stephan, U., Erlangen 1677

Stickl, H., München 1425

Stille, W., Frankfurt 1518, 1816, 2226, 2484

Stoeckenius, Hamburg 2622*

Stoll, Mannheim 2253*

Stoll, L., Frankfurt 1816

Stralau, J. 2301*

Straub, Marburg 1898*

Straub, P. W., Zürich 1885, 2212

Streicher, E., Stuttgart 1441, 1820

Strnad, F. 2184*

Strohmeyer, G., Marburg 1577, 1842

Studer, Bern 2528*

Stutz, Freiburg 2184*

Stutz, L. 1427"

Südhof, H., Gehrden/Hannover 1468*, 2007, 2065*

Suschke, J., München 2289

Suwelack, M., Hamburg 2631

Sweet, W. H. 2361*, 2622*

Sykes, M. K. 2410*

Szasz, G., Gießen 1911

T

Talke, H., Freiburg i. Br. 2284

Talke, H., Mainz 1917

Tauber, Frankfurt a. M. 2300*

Tenner, R. 2460

Thaler, H., Wien 1544, 1842

Thaler, Wien $1627^{*}$

Thews, G. 1707*

Thiele, H., München 2488

Thomas, P. 1587

Thomsen, K. $1627^{\circ}$

Thurn, P. 2704:

Tiedt, N. 1953*

Tilsner, V. $1427^{*}$

Tilsner, V., Hamburg 1604

Tismer, R., Köln-Lindenthal 2135,2547

Todorow, S., Tübingen 1418

Tornow, P., Oldenburg 1503

Trüb, C. L. 2301:

Truninger, B., Bern 1695

Tschöpe, E., Kiel 1426

Türk, E., Teterow 2554

Tyrrell, D. A. J. 1843*

\section{$\mathrm{U}$}

Uehlinger, A., Schaffhausen 1640 Uehlinger, E., Zürich $\mathbf{2 1 4 0}$ Uldall, Birgit, Flensburg 1453 Ullman, U., Tübingen 2399

Ulmer, Bochum 2129*

Ulmer, W. T., Bochum 2375

Umbach, Berlin 2361*, $2622^{*}$

Umbach, Siegen $1426^{*}$

Umbach, W., Berlin 2525

Umek, H., Wien 1574, 2004

Urthaler, F., Zürich 2290

Frhr. v. Ungern-Sternberg, A.,

Mainz 2118

V

Vahlensieck, W., Bonn-Venusberg 2381

Valentine, G. H. $2622^{*}$
Stern, C. 2301*

Verbeck, B, Münster $\mathbf{1 7 6 1}$

Viamonte, M. 2704:

Vido, I., Göttingen 1633, 1637

Vido, I., Göttingen 2215, 2220

Vieten, H. 2184*

Vieten, H., Düsseldorf 1593

Virchow, Chr., Davos-Wolfgang 2508

Vivell, O., Karlsruhe 1626, 1771

Vogel, F. 2183*

Vogler, E. 2704:

Vogt, O., Marburg/Lahn 2155

Volles, E., Göttingen 2682

Vollmar, J., Heidelberg 2315

Vorlander, K.-O., Aachen 2063

van Voss $1587^{*}$

Voss, D., Marburg/Lahn 2525

W

v. Waechter, R., Berlin 2421

Wässer, St., Leipzig 1491, 1928

Wagenhäuser, F. J., Zürich 1579

Wagner, A., Nürnberg $\mathbf{1 8 5 6}$, 2033, 2097

Wager, H., Münster 1877

Wagner, J., Bonn 1717

Waldenström, J. G. 1898*

Waldhäusl, W., Wien 2028

Wannagat, L. 1627*

Wannagat, L., Bad Mergentheim 2120

Warnatz, H., Erlingen 2444

Watson, J. D. 2410*

Weber, A., Marburg/Lahn 2206

Weber, G., Zürich 2442

Wechsler, W. 2527*

de Weck, A. L., Bern 2627

Wedell, J., Aachen 2539

Wegmann, T., St. Gallen/Schweiz. 1992

Wehner, H., Tübingen 1673

Weidmann, P., Bonn 2685

Weinstein, A. S. 2622"

Weisbecker, L., Kiel 2657

Weiss, K. E. 2662"

W'eitbrecht, Bonn 1953:

Weller, S., Freiburg i. Br. 2705

Wellmann, New York 2185* $2528 * 2651^{*}$

Wellman, P., Essen 1566

Welsch, A. 2360"

Welsch, A., Isny!Allgäu 2438

Wenger, Wien 2360"

Wenger, R., Wien 1537, 1539

Wenz, W., Heidelberg 1483

Werle, E. 1668*

Werner, M., Pinneberg 1802

Werner, U., Bochum 2375 
Wilhelmi, J., Neunkirchen-Kohlhof/Saar 1959

Wille, L., Heidelberg 2223

Wilmanns, Tübingen 2065*

Windorfer, A., Erlangen-Nürnberg 2071

Winckelmann, G., Freiburg 1752

Wink, K., Essen 2160

Winkle, Hamburg 1628*

Winkler, K. 2461"

Winter, J., Heidelberg 2223

Wiontzek, H., Geesthacht 2356, 2568

Wirth, W., Zürich 2395

Wirz, P., Zürich 2579
Witte, P., Hamburg 1978

Witzleb, E. $1707^{*}$

Wohlenberg, H., Heidelberg 2163, 2240

Wolf, H., Göttingen 1996

Wolter $\mp$, Heidelberg $1628^{*}$

Wolter, M., Berlin 2102

Wormser, P., Zürich 2618

Würdinger, H., Esslingen 1688

Würz, H., Stuttgart 1441

Wuketich, St., Wien 2107

Wullstein, H. L. 1773*

Wullstein, Würzburg 2064*

Wuth, K., Berlin 1704 R, 1705 R, 2244 R, 2454*
$\mathrm{Y}$

Yasargil, M. G., Zürich 1496

$\mathrm{Z}$

Zander, J. 1627*

Zdansky, E. 1952:

Zechner, G. 2704*

Zeitler, E. 2704*

Zeman, N., Homburg/Saar 1497

Zemplényi, T. 2129*

Zerbin-Rüdin, E., München 2519
Ziegler, M., Heidelberg 1765

Ziegler, M., Heidelberg 1765

Ziegler, W. H., Zürich $\mathbf{2 6 7 8}$

Zilliken, F. 2527"

Zimmermann, U., Wittenberge/ DDR 1422

Ziolko, H.-U. 2252*

Zöllner, Freiburg i. Br. 1773*

Zöllner, F. 2183*

Zöllner, N., München 1933, 2652

Zorn, H., Tübingen 1692

Zülch, K. J. $2573^{*}$

Zukschwerdt, Hamburg 2662*

Zuppinger, A. 2184*

Zutz, H.-U., Bad Nauheim 2569

\section{Personalnachrichten}

\section{Berufungen, Lehraufträge, Ernennungen}

Ahrens, G.,Hamburg 2187

L'Allemand, H., Gießen 2068

Andres, K. H., Heidelberg 2255

Antoni, H. T., Frankfurt 1589

Apitz, J., Tübingen 2576

Appels, A., Göttingen 1846

Ascher, F., München 1778

Bässler, R., Mainz 1550

Bättig, K., Zürich 1710

Battegay, R., Basel 1670

Baumgarth, F., München 2624

Bay, V., Hamburg 2624

Bender, F., Münster 1510

Benthe, H. P., Lamburg 1630

Berchtold, R., Basel 1589

Berg, G., Erlangen-Nürnberg 1509

Berg, St., Göttingen 1550

Berger, J., Basel 1670

Best, W., Tübingen 1550

Biesalski, P., Mainz 1630

Bingas, B., Berlin 2575

Bister, W., Gießen 2412

Bönicke, R., Kiel 2187

Borchers, H. G., München 2011

Brecht, K., Ulm 2576

Brehm, G., Mainz 2131

Brendel, W., München 1589

Breuninger, H., Tübingen 1550

Briesalski, P., Mainz 1630

Brossmer, R., Heidelberg 1589

Büchs, H., Bonn 2530

Bushe, K.-A., Göttingen 1550

Campana, L., Bern 1670

Carlson, S., Mainz 2131

Clotten, R., Freiburg 2187

Conze, W., Heidelberg 1900

Cramer, F., Göttingen 1470
Decher, H., Bonn 2575

Delbrück, A., Hannover 1550

Dettli, L., Basel 1589

Dhom, G., Saarbrücken 1510

Ditschuneit, H., Ulm 1589

Döll, N., Würzburg 2624

Dölle, W., Marburg 2255

Dohrmann, R. E., Bonn 2363

Doll, E., Freiburg 2707

Drexel, H., München 2011

Dubach, U. C., Basel 1589

Düx, A., Bonn 1846

Dziallas, P., München 2363

Ebert, G., Göttingen 1846

Eckel, P., Göttingen 1589

Einbrodt, H.-J., Aachen 1710 , 2011

Falke, D., Mainz 2131

Fasold, H., Frankfurt 1589

Fiebiger, N., Erlangen-Nürnberg 1509

Fischer, J., Mainz 2131

Fleckenstein, A., Freiburg 1470, 2707

Fleisch, H., Bern 2131

Fleischer, K., Hamburg 1630

Flemming, K., Freiburg 2530

Franke, D., Heidelberg 2255

Fratz, Rosemarie, Düsseldorf 2411

Freyschmidt, P., Berlin 2464

Fries, G., Saarbrücken 1047

Frik, W., Aachen 1470

Fritz, K. W., Bonn 2623

Frowein, R., Köln 2624

Georg, H., Heidelberg 1589

Gieseking, Rotraud, Münster 2363

Fritz, K. W., Bonn 2623

Glaninger, J., Wien 1470

Gloor, F., Basel 2363

Gögler, E., Heidelberg 2255
Goerke, H., München 2303

Gött, U., Bonn 1955

Gottheiner, V., Berlin 2664

Grabner, G., Wien 1470

Gramlich, F., Mainz 2131

Grüsser, O.-J., Berlin 1470

Gupta, D., Tübingen 2576

Haberich, F.- I., Marburg 2530

Habermehl, K.-O., Berlin 2664

Haider, M., Wien 1550

Hamelmann, H., München 1510

Harbauer, H., Frankfurt 1630

Harlfinger, $H$., Tübingen 1670

Hartung, W., Münster 1710

Hauke, H., Bonn 2411

Haunfelder, D., Münster 1710

Hauser, W., Bonn 1955

Hecker, W. Ch., München 1589

Heckmann, K. D., Saarbrücken 1847

Heckmann, U., Saarbrücken 1429

Heimpel, H., Ulm 1589

Heipertz, W., Frankfurt 2464

Herfert, O., Mainz 2131

Hess, F., Marburg 2011, 2303

Hienz, H.-A., Essen 1778

Hilber, H., München 2707

Hild, R., Heidelberg 1589

Hilger, H. H., Bonn 2623

Hille, H., Bern 2131

Hinrichsen, K., Tübingen 1589

Hipp, E., München 2707

Hippius, H., Berlin 2663

Hirche, H., Düsseldorf 1846

Hirsch, W., München 2624

Hochrein, H., Würzburg 1550 , 2530

Höhling, H.-J., Münster 1510

Hofmann, M., Tübingen 1550

Hohmann, D., Erlangen-Nürn-

berg 1509
Hohorst, H.-J., Marburg 2624

Holstein, F., Hamburg 1900

Holsten, D. R., Hannover 2464

Holtmeier, H.-J., Freiburg 1900

Huerkamp, B., Göttingen 1846

Hürlimann, A., Bern 1710

Huhnstock, K.-H., Heidelberg 1589

Ippen, H., Düsseldorf 1846

Jagdschian, V., Berlin 1470

Jahrmärker, H., München 2363

Jaisle, F., Würzburg 2363

Jörgensen, G., Göttingen 1846

Juchems, R., Würzburg 2624

Kärcher, K.-H., Wien 1470

Kanz, E., München 1630

Karcher, G., Bonn 2464

Keller, H.-L., Erlangen-Nürnberg 1509

Kemper, F., Münster 2363

Kerstein, G., Frankfurt 2363

Kersting, G., Bonn 1589

Kessel, M., Berlin 2664

Kesseler, K., Bonn 1955

Kindler, H., Berlin 2664

Kirchner, E., Hannover 1710

Klaus, W., Mainz 2131

Kleibel, F., Heidelberg 2255

Kleihauer, E., Ulm 1590, 2576

Kleinsasser, O., Köln 2624

Klosterkötter, W., Essen 1778

Klütsch, K., Würzburg 2664

Koch, D., Düsseldorf 1846

Köle, H., Wien 1470

Koepchen, H.-P., Berlin 2664

Koeppen, S., Braunschweig 2464

Kollwitz, A. A., Berlin 2664

Kovac, W., Wien 1470

Kraft, E., München 1900

Kremer, K., Essen 1778

Kröger, H., Freiburg 2707

Krüger, E., Bonn 1955 
Kubicki, St. K., Berlin 2011

Kucher, R., Wien 1470

Küster, F., Essen 1778

Kugler, J., München 2011

Kurz, H., München 1778, 2576

Kutz, E., Gießen 2412

Lange, F. Ch., Würzburg 2624

Langendörfer, G., Bonn 2464

Langendorf, H., Mainz 2131

Lautenbach, L., Erlangen-Nürnberg 1510

Lehnhardt, E., Hamburg 1955

Lévy, A., Basel 2363

Lierse, W., Hamburg 2068

Lindner, E., Regensburg 1630

Lissner, J., München 1778

Loch, W., Tübingen 1550

Löhr, E., Essen 2664

Lüscher, E. F., Bern 2131

Mallach, H. J., Tübingen 1670

Marghescu, S., München 2530

Marquardt, E., Heidelberg 2776

Marti, H. R., Basel 1589

Martin, H., Frankfurt 2363

Marxkors, R., Münster 1710

Matussek, P., München 1429

Matzker, J., Mainz 1470

Maurath, J., Freiburg 2707

Mayer, K., Tübingen 1670

Messmer, E., Heidelberg 2255

Mössdorf, K., Bonn 1955

Mohing, W., Erlangen-Nürnberg 1509

Mohr, W., Hamburg 1630

Mosler, K. H., Würzburg 2530

Müller, E., Hamburg 1630

Miiller, G., Göttingen 1846

Müller, G. H., Bonn 2464

Mürtz, R., Düsseldorf 1846

Müsebeck, K., Saarbrücken 1510

Nasemann, Th., Frankfurt 2255

Naumann, H., Berlin 1470

Nusser, E., Heidelberg 1900

Ober, K. G., Erlangen-Nürnberg 2707

Oberdorf, O., Düsseldorf 2363

Oberhoffer, G., Bonn 2464

Otto, H., Erlangen-Nürnberg 1509

Otto, H., Münster 2068

Otto, K., Bonn 1955

Pabst, W., München 1778

Palm, D., Frankfurt 2363

Passow, H., Saarbrücken 1510 , 1846

Pels-Leusden, F., Kiel 2664

Petschke, H., Wien 1470

Pfeiffer, K., Münster 2068

Pfeiffer, R.-A., Münster 2707

Piper, J., Göttingen 1846

Rasch, W., Köln 2624

Reiffenstuhl, G., Münster 2363

Reiffenstuhl, P., Graz 1778

Reimer, F., Kiel 1670

Reisert, P. M., Göttingen 2255

Remmele, W., Mainz 1900

Remmer, H., Tübingen 2576

Riegel, K., München 2576

Rind, J., Gießen 2587

Ritze, H., Hamburg 2303

Röckl, H., Würzburg 1550

Rohr, H., Bonn 2530

Rollhäuser, H., Münster 1900

Rossberg, G., Frankfurt 1470

Rost, A., Tübingen 2576

Rutishauser, G., Basel 1670

Sachse, H. E., München 2011

Sachsenmaier, W., Heidelberg 1589

Scheidegger, S., Basel 1670
Scheppokat, K.-D., Hamburg 2664

Scheunemann, H., Düsseldorf 1846

Schipperges, H., Heidelberg 2575

Schlegel, H.-J., Saarbrücken 2412

Schmidt, H., Erlangen-Nürnberg 1509

Schmidt-Matthiesen, $\mathrm{H}$. Göttingen 1846, 2255

Schmitz, W., Heidelberg 2576

Schneweiss, K.-E., Bonn 2530

Schrader, K., Gießen 2623

Schrader, K.-E., Frankfurt 1589

Schrappe, O., Marburg 2530

Schriefers, H., Ulm 2363

Schriefers, K. H., Bonn 2464

Schubart, A., Mainz 2131

Schüssler, R., Saarbrüicken 1510

Schwarz, H., Wien 1710

Schweitzer, H., Düsseldorf 1846

Seitz, D., Göttingen 1470

Sickinger, K., Göttingen 1846

Sigel, A., Erlangen-Nürnberg 2623

Simon, C., Kiel 1589

Sökeland, J., Saarbrücken 2412

Sonnabend, E., München 1589

Spann, W., München 2576

Spuler, H., Würzburg 2624

Staehelin, M., Bern 1710

Stark, G., Erlangen-Nürnberg 1510

Steigleder, G. K., Köln 2624

Steinbereithner, K., Wien 1470

Stoboy, H., Berlin 2664

Stochdorph, O., München 1589

Stöber, W., Münster 2068

Storck, H., Zürich 2068

Stralau, J., Bonn 2363

Strassburg, M., Düsseldorf 1846

Strauss, G., Mainz 2131

Strotzka, H., Wien 1710

Strupler, W., Bern 2131

Stutz, E., Freiburg 1470

Taupitz, A., Saarbrücken 2363

Tellenbach, H., Heidelberg 1900

Thoenes, W., Würzburg 2624

Thurau, K., München 1589

Trautwein, W., Heidelberg 1900

Trendelenburg, F., Saarbrüicken 2664

Tritsch, H., Köln 1670

Uehleke, H., Tübingen 1550

Vahlensieck, W., Bonn 1630

Valentin, H., Erlangen-Nürnberg 1900

Vido, I., Göttingen 1846

Waller, H. D., Tübingen 2576

Walter, P., München 1589

Wartenberg, H. A., Basel 2363

Waubke, T., Essen 1778

Weber, W., Frankfurt 2363

Weinschenk, C., Marburg 2412

Weis, K. H., Würzburg 1550

Weise, W., Düsseldorf 1846

Weiser, P., Münster 1670

Weishaar, J., Erlangen-Nürnberg 1509

Weitbrecht, H. J., Bonn 1470

Wellauer, J., Zürich 2068

Weller, S., Freiburg 2011

Wewalka, F., Wien 1470

Wiemers, K., Freiburg 1470

Wigand, R., Saarbrücken 1847

Wildhirt, E., Göttingen 2255

Wolf, D., Heidelberg 2576

Wüthrich, R. J., Basel 1670

Wulf, K.-H., Hannover 2412

Wyss, D., Würzburg 2530
Zander, J., Heidelberg 1589

Zander, J., Marburg 2255

Zenker, W., Wien 1670

Zimmer, F., München 2363

Habilitationen,

Umhabilitationen,

Dozenturen

Albers, H., Mainz 2131

Allert, M. L., Saarbrücken 1847

Andrée, G., Saarbrücken 1847

Appels, A., Göttingen 1470

Arnold, G., Düsseldorf 1846

Aziz, O., Berlin 2664

Bartels, H., Lübeck 2187

Bassenge, E., München 2011

Baumann, F., Saarbrücken 2412

Baumgartl, F., München 1900

Baurmann, H., Bonn 2363

Bayindir, S., Gießen 2412

Benzer, H., Wien 1710

Berg, D., Frankfurt 1589

von Berg, W., Göttingen 1846

Berle, P... Hamburg 1955

Bernett, P., München 1900

Böhmer, D., Frankfurt 2363

Bollinger, A., Zürich 1590

Born, W., Freiburg 2131

Breckwoldt, M., Hamburg 1955

Brune, G., Würzburg 1550

Buchwald, W., Mainz 2131

Büchner, M., Düsseldorf 1846

Buse, H., Göttingen 1846

Ciompi, L., Lausanne 2131

Cramer, H., Freiburg 2131

Denk, R., Mainz 2131

Dieckmann, G., Saarbrücken 1847

Diederich, K.-W., Lübeck 2624

Distler, G.-A., Mainz 2131

Dransfeld, H., Düsseldorf 1846

Dudeck, J., Mainz 2131

Ebner, H., Wien 1710

Ewe, K., Mainz 2131

Eyrich, K., Freiburg 2131

Fenner, A., Lübeck 2187

Forssmann, W. G., Genf 1710

Gerstenberg, E., Berlin 1470

Gillich, K. H., Hannover 1470

Graeff, H., Heidelberg 1589

Grimmer, H., Mainz 2131

Grossmann, D., Frankfurt 2363

Grüter, H., Münster 1710

Grützner, A., Mainz 2131

Gschwend, N., Zürich 1510

Güirich, H.-G., Lübeck 2624

Haage, H., Kiel 2187

Hadorn, H.-B., Bern 2068

Haelpap, B., Marburg 1710

Harbauer, G., Saarbrücken 1847

Hartenbach, W., Mainz 2131

Heinz, F., Hannover 1470

Hellner, K., Hamburg 1955

Henn, R., Freibùrg 2131

Henselmann, L., München 1900

Hermanek, P., Erlangen-Nürnberg 1510

Herrmann, H.-D., Saarbrücken 2363

Herschlein, H.-J., Tübingen 1550

Hiersche, H.-D., Mainz 2131

Hirsch, W., München 1900

Hoffmann-Daimler, S., Tübingen 1550

Hollmann, K. H., Saarbrücken 1847

Hoppe, I., Berlin 2664

Ivankowic, St., Freiburg 2131

Jacobi, K., Köln 1630

Jantzen, K., Göttingen 1470

Jovanovic, U., Würzburg 2624

Kaffarnik, H., Marburg 1710

Kalbhen, D.-A., Bonn 2255

Kessler, W., Mainz 2131

Ketz, E., Zürich 1670

Kirchhof, J., Bonn 1955

Kladetzky, J., Düsseldorf 1846

Klinke, R., Berlin 2664

Klotter, H.-E., Mainz 2131

Kruppen, R., Bonn 2255

Kössling, F. K., Mainz 2131

Kohler, E., Zürich 2363

Kornhuber, B., Frankfurt 1589

Kotscher, E., Wien 1710

Kowalewski, S., Bonn 1710

Kraus, H., Münster 2707

Kreysel, H.-W., Hamburg 1955

Kriehuber, E., Wien 1670

Krone, H.-A., Erlangen-Nürnberg 2011

Kuhn, D., Heidelberg 1589

Lange, F. Ch., Würzburg 1630

Lemmingson, W., Tübingen 2576

Liebhardt, E., Freiburg 2131

Linnen, H. J., Frankfurt 2363

Lösch, G., Lübeck 2624

Lorbacher, P., Bonn 2575, 2623

Lorentz, K., Lübeck 2624

Lorenz, W., München 2011

Loskant, G., Saarbrücken 1847

Ludwig, H.-J., Lübeck 2624

Luhr, H.-G., Hamburg 1955

Lungershausen, E., Bonn 2363

Luthe, R., Saarbrücken 1847

Mach, K., Wien 1470

Maurermayer, W., München 1630

Meier, G., Lübeck 2624

Meiser, J., Saarbrücken 1847

Meyer, D., Tübingen 1670

Mildenberger, $H$., Tübingen 1550

Moeschlin, S., Zürich 1710

Moser, K., Wien 2011

Müller, H., Frankfurt 2363

Müller-Oerlig, B., Göttingen 1846

Müller, W., Würzburg 2363

Niedobitek, F., Lübeck 2624

Nitzke, H.-R., Frankfurt 2363 
Schewe, E. G., Frankfurt 2363 Schimrigk, K., Hamburg 1955 Schmidt, H., Hamburg 1955 Schobinger, R., Bern 2068 Schoner, W., Göttingen 1846 Schröder, E., Düsseldorf 1846 Schubert, R. H., Frankfurt 2363 Schütz, R.-M., Lübeck 2624 Schuster, W., Erlangen-Nürnberg 1510

Specht, W., Saarbrücken 1847

Stefenelli, N., Wien 2011

Steuer, W., Tübingen 2576

Stewart, Ute, Würzburg 2624

Stiebitz, R., Wien 2363

Svejear, J., Frankfurt 2363

Teubner, E., Lübeck 2624

Theissing, J., Mainz 2131

Ueberberg, U., Saarbrücken 2255

Ucberg, H., Saarbrücken 1847

Uhlmann, K., Kiel 2187

Vahl, J., Münster 1710

Vittali, H., Köln 1630

Wahl, P., Heidelberg 1589

Wand, H., Kiel 2187

Warecka, K., Lübeck 2624

Wepler, W., Göttingen 1846

Westhues, M., München 2011

Winkelmann, O., Frankfurt 2363

Wollert, U., Mainz 2131

Wulff, E., Gießen 2412

Zysno, E. A., Gießen 2412

\section{Korrespondierendes Mitglied, Gesellschaften, Auszeichnungen}

Altmann, H.-W., Würzburg 2363 Arnold, M., Tübingen 2576

Ascher, F., München 1589

Battegay, R., Basel 2363

Bay, E., Düsseldorf 1846

Beck, Ch., Freiburg 2187

Becker, V., Berlin 2663

Bernhard, W., Basel 2707

Bockelmann, P., München 1710

Böhlau, V., Frankfurt 2363

Braun, H., Würzburg 1550

Braun-Falco, O., München 2011, 2576

Brecke, F., St. Blasien 1429

Brittinger, G., Bochum 1470

Budelmann, G., Hamburg 1900

Butenandt, A., München 2363

Choné, B., Heidelberg 1589

Derra, E., Düsseldorf 1630, 1846

Doenecke, F., Saarbrücken 2412

Dukor, B., Basel 2707

Franke, K., Bad Lauterberg/Harz 1510

Friederich, H. C., Tübingen 2576

Fröhlich, E., Tübingen 2576

Frohmüller, H., Würzburg 2363

Gasser, F., Basel 2363

Gerson, W., Göttingen 2068

Giese, W., Münster 2707

Goecke, H., Münster 2707
Götz, H., Essen 1710, 2255

Greither, A., Düsseldorf 1846

Gruber, U. F., Basel 2363

Haas, R., Freiburg 2707

Habs, H., Bonn 2707

Hadorn, E., Zürich 2011

Hammerstein, J., Berlin 2664

Hadorn, W., Bern 1509

Hamperl, H., Bonn 2363, 2464

Haselmann, H., Tübingen 2576

Holtmeier, H.-J., Freiburg 1470 , 2130,2707

Jadassohn, W.-E., Genf 2011

Janz, D., Heidelberg 2575

Jung, R., Freiburg 2530

Karlson, P., Marburg 2624

Keidel, W.-D., Erlangen-Nürnberg 1509

Kellerer, A. M., München 2011

Kirchhoff, H., Göttingen 1846

Kleine-Natorp, H.-E., Dresden 2464

Knüttgen, H. J., Tübingen 2576

Kretschmer, W., Tübingen 1550

Künzer, W., Freiburg 2707

Laves, W. München 1589

Lax, H., Berlin 2663

Lemperle, G., Freiburg 2707

Levine, R., Ulm 1589

Limburg, H., Saarbrücken 1846

Lipmann, F., Hannover 1550

Loeschke, A., Berlin 2663

L.orenz, E., Graz 2623

Maassen, W., Essen 1710

Mahlo, A., Hamburg 1710

Mall, G., Tübingen 2576

Markoff, N., Zürich 2530

Martini, G. A., Marburg 2664

Mayer, J. B., Saarbrücken 1846

Meessen, H., Düsseldorf 1846

Mehnert, H., München 2624

Merten, R., Düsseldorf 1846

Mikat, B., Berlin 1589

Mittelmeier, H., Saarbrücken 1846

Mueller, B., Heidelberg 1589

Muth, H., Saarbrücken 1510

Nagel, R., Berlin 2663

Oehme, J., Marburg 2624

Freiherr v. Oldershausen, H.-F., Tübingen 1630

Richards, R. K., Hamburg 1630

Schadewaldt, H., Düsseldorf 1846

Schirren, C., Hamburg 1846

Schlegel, H.-G., Göttingen 1630

Schmidt-Matthiesen, $\mathrm{H}$. Göttingen 1470

Schneider, W., Tübingen 2576

Schnyder, U. W., Heidelberg 2624

Schubert, R., Erlangen-Nürnberg 2411

Schulte, W., Tübingen 2707

Sewering, H. J., München 1470

Siede, W., Frankfurt 2363, 2664

Spechter, H. J., Tübingen 2576

Staehler, W., Tübingen 1550

Stender, A., Berlin 2664

Teller, W., Ulm 1589

van Thiel, H., München 1589

Uehlinger, E., Zürich 1710

Vieten, H., Düsseldorf 1846

Wachsmuth, W., Würzburg 2530

Wagner, R., München 1778
Walther-Büel, H, Bern 2011

Westphal, O., Freiburg 2707

Wollheim, E., Würzburg 2363, 2624

Zimmermann, W., Saarbrücken 1847

Zuppinger, A., Bern 2187

\section{Entpflichtungen}

von Braunsbehrens, H., München 2011

Doenecke, F., Saarbrïcken 2412

Hellner, H., Göttingen 1846

Kreibig, W., Saarbrüicken 2412

\section{Geburtstage}

von Albertini, A., Zürich 1670

Alken, C.-E., Saarbrücken 2131

Blechschmidt, E., Göttingen 2363

Bode, H.-C., Göttingen 1778

Bordasch, F., Hamburg 1900

Bürki, E., Basel 2464

Dirscherl, W., Bonn 2464

Dittmar, O., Heidelberg 1510

Eckert-Möbius, A., Halle 2530

Erbslöh, J., Kiel 2011

Fabian, H., Hamburg 2530

Flesch-Thebesius, M., Frankfurt 1429

Forssmann, W., Düsseldorf 1770

Franz, G., Hamburg 1955

Gagel, O., Erlangen-Nürnberg 1429

Gasteiger, H., Berlin 2464

Gerke, J., Düsseldorf 1846

Gertler, W., Berlin 2530-

Goldmann, H., Bern 2411

Hackenbroch, M., Köln 1429

Hertwig, P., Halle-Wittenberg 2011

Hett, J., Erlangen-Nürnberg 1670

Keil, R., Rostock 1778

von Keiser, D., Heidelberg-

Mannheim 2363

von Keyserlingk, H., Jena 1778

Kroetz, Ch., Hamburg 2575

Labes, R., Bonn 1550

Lang, F. J., Innsbruck 1429

Lange, M., München 1550

Linzbach, J., Göttingen 2707

Mahlo, A., Hamburg 1470

Mannherz, C., Heidelberg 2412

Matzen, P.-F., Leipzig 2131

Meessen, H., Düsseldorf 1846

Mörl, F., Halle-Wittenberg 2303

Müller, M., Bern 1778

Netter, H., Kiel 1470

Oberniedermayr, A., München 2303
Oppolzer, R., Wien 1955

Pape, R., Wien 1956

Pischinger, A., Wien 1470

Psenner, L. B. Wien 2011

Rabe, F., Hamburg 1900

Reichling, W., Würzburg 1778

Rosenthal, W., Berlin 1846

Schall, L., Hamburg 1510

Scheid, W., Köln 1510

Schinzel, A., Innsbruck 2303

Schliephake, E., Würzburg 1670

Schmidt, K., Bonn 2255

Schmuziger, P., Zürich 1847

Schneider, Max, Köln 2187

Scholz, W., München 2624

Schürmeyer, A., Köln 2530

Schwartz, Ph., Frankfurt 1510

Spath, F., Graz 2530

Stutte, H., Marburg 1589

Tappeiner, J., Tübingen 2707

Uehlinger, E., Zürich 1630

Veit, O., Köln 2187

Volkmann, J., Greifswald 2011

von Volkmann, R., Tübingen 1510

Voss, H., Jena 2131

Weber, A., Gießen 1589

Weinig, E., Erlangen-Nürnberg 1429

Wiesmann, E., Zürich 2576

Wildführ, G., Leipzig 1778

Wilke, G., Gießen 1470

Wolf, H., Würzburg 2011

Zinner, H., Wien 1956

Zipf, K., München 2707

\section{Todesfälle}

Denecke, K., Münster 1510

Diehl, K., Frankfurt 2412

Erggelet, H., Göttingen 2303

Fischer, W., Kiel 2068

Gross, K., Erlangen-Nürnberg 2255

Harff, J., Hamburg 2464

Heilmeyer, L., Ulm 2012

Hoff, H., Wien 1900

Köbcke, H., München 2303

Kohlrausch, A., Tübingen 1630

Lendle, L., Göttingen 1900

Lichtenauer, F., Hamburg 2364

Saller, K., München 2364

Schirren, C. G., Marburg 1670

Schultz, W., Hamburg 2364

Staehelin, J., Basel 1670

Thörner, W., Bonn 2255

Thomas, K., Göttingen 2068

Uhlenbruck, P., Köln 2412

Valentin, B., Hannover 2364

von Verschuer, O., Münster 2255

Vogler, P., Berlin 2531

Wassermann, F., Donners Grove, Ill./USA 1470

von Werz, R., München 2576

Witebsky, E., Buffalo, N.Y./USA 2708 


\section{Aktuelle Diagnostik}

Redaktion:

Prof. Dr. H. Hornbostel, Hamburg

Prof. Dr. W. Kaufmann, Tübingen

Prof. Dr. W. Siegenthaler, Bonn

\section{Die diagnostische Wertigkeit des Trijodthyronin-Tests}

Die verschiedenen Modifikationen der Trijodthyronin$\left(\mathrm{T}_{3^{-}}\right)$in-vitro-Tests sind indirekte Verfahren zur Ermittlung des Schilddrüsenhormongehaltes im Serum. Der Prototyp dieser Methoden ist der von Hamolsky und Mitarbeitern (1) angegebene Trijodthyronin-ErythrozytenAufnahmetest.

Die Schilddrüsenhormone Trijodthyronin und Thyroxin sind im Blut zu über 99\% an Plasma-Eiweißkörper einerseits und Erythrozyten andererseits gebunden, so daß der freie, biologisch wirksame Hormongehalt nur Bruchteile von 1\% ausmacht. Folgendes Schema erleichtert das Verständnis des Prinzips der zu besprechenden Methoden:

$$
\begin{array}{lll} 
& + \text { Plasmaproteine } & \rightleftharpoons\left[\begin{array}{l}
\text { Plasmaprotein-Schild- } \\
\text { drüsenhormon-Komplex }
\end{array}\right] \\
\begin{array}{l}
\text { Freies } \\
\text { hchilddrüsen- } \\
\text { hormon } \\
\end{array} & + \text { Erythrozyten } & \rightleftharpoons\left[\begin{array}{l}
\text { erythrozytengebundenes } \\
\text { Schilddrüsenhormon }
\end{array}\right]
\end{array}
$$

$$
(99 \%)
$$

Trennt man vom Blut eines Patienten die Eiweißkörper ab und bestimmt in ihnen chemisch den Jodgehalt, so erhält man den $\mathrm{PB}^{127} \mathrm{I}-$ Wert. Auf die Störanfälligkeit und Fehlerquellen dieser Bestimmung des proteingebundenen Jods kann hier nicht näher eingegangen werden $(2,3)$. -

\section{P. C. Scriba}

2. Medizinische Universitätsklinik München (komm. Direktor: Prof. Dr. M. M. Forell), Abteilung für Endokrinologie (Leiter: Prof. Dr. K. Schwarz)

Hamolsky und Mitarbeiter (1) inkubierten radioaktives Trijodthyronin mit Blut und maßen die Verteilung der Radioaktivität zwischen Plasma-Eiweißkörpern und Erythrozyten. Da die Kapazität der schilddrüsenhormonbindenden Plasma-Eiweißkörper nur begrenzt ist, fanden sie bei erhöhtem endogenem Schilddrüsenhormonspiegel mehr radioaktives Trijodthyronin an den Erythrozyten.

Heute werden statt der Erythrozyten aus technischen Gründen Ionenaustauschharze, Dextran-Gel oder andere Adsorbentien benützt. Dabei kann man entweder zuerst radioaktives Trijodthyronin mit Serum inkubieren und die Adsorbentien nachträglich einwirken lassen oder markiertes Trijodthyronin, Serum und Austauscherharze gleichzeitig inkubieren. Gemessen wird in jedem Fall am Ende der Anteil des markierten Schilddrüsenhormons, der nach der Inkubation noch an Eiweißkörpern gebunden ist, und derjenige Anteil, der zum Beispiel vom Dextran-Gel adsorbiert wurde, und zwar in Prozent der insgesamt zugesetzten Radioaktivität. Es handelt sich also nicht um eine direkte Bestimmung des freien Anteils der Schilddrüsenhormone, wie inkorrekt häufig zu lesen ist, sondern um eine Ermittlung der proteingebundenen und der beispielsweise an Austauscherharze gebundenen (»freien «) Radioaktivität. Wird der Wert in Prozent der zugesetzten Aktivität angegeben, so muß in jedem Falle anhand einer ge- 
nügend großen Gruppe von Normalpersonen mit statistischen Methoden der Normalbereich ermittelt werden. Dieser Normalbereich hängt ganz von Volumen und Kapazität des Serums einerseits und Menge und Adsorptionsfähigkeit von Dextran-Gel oder Austauscherharz andererseits ab. Die Dimensionen der verschiedenen Inkubationsansätze sind also laboratoriumseigen. Aus diesem Grunde geben manche Autoren heute der Verwendung eines Quotienten den Vorzug, bei dem der proteingebundene Anteil eines unbekannten Serums durch den proteingebundenen Anteil eines Kontrollserums dividiert wird (TBI). Allen Formen des $\mathrm{T}_{3}$-in-vitro-Tests, sei es daß man eine hauseigene Methode oder eine kommerzielle Testpackung verwendet, ist gemeinsam, daß die Qualität des heute meist mit ${ }^{125} \mathrm{Jod}$ markierten Trijodthyronins und die Adsorptionsfähigkeit des Austauscherharzes oder des DextranGels zum Teil erheblichen Schwankungen unterworfen sind, ganz abgesehen davon, daß Temperatur, $\mathrm{pH}$, Ionenstärke und andere Faktoren einen Einfluß haben. Wie für alle klinisch-chemischen Verfahren gilt also auch für den $\mathrm{T}_{3}$-in-vitro-Test, daß eine tägliche Qualitätskontrolle durch Kontrollseren unerläßlich ist.

Diagnostische Treffsicherheit: Die Werte des $\mathrm{T}_{3}$-Tests einer repräsentativen Gruppe von Kontrollpersonen und unausgewählter Gruppen von Patienten mit gesicherter Schilddrüsenüberfunktion oder Schilddrüsenunterfunktion zeigen jeweils eine Verteilung, die einer Gaußschen Kurve entspricht. Eine optimale Treffsicherheit wäre gegeben, wenn diese drei Gauß-Kurven nicht überlappen. Das ist theoretisch nicht zu erwarten und praktisch auch nicht der Fall. Unsere eigenen Erfahrungen zeigen eine Treffsicherheit des $\mathrm{T}_{3}$-Tests bei der Hyperthyreose von $90-95 \%$ und eine Treffsicherheit von nur etwa $50 \%$ bei der Hypothyreose $(3,4)$. Bei Hyperthyreose fehlt die Erhöhung des sogenannten freien Trijodthyronin- ${ }^{125}$ Jods also höchstens in $10 \%$.

Störfaktoren: Das Problem der Treffsicherheit kann jedoch nicht unabhängig von den Störfaktoren gesehen werden. Störfaktoren führen zu falsch-positiven Ergebnissen, also zu einer falsch interpretierbaren Erniedrigung

oder Erhöhung der Werte des sogenannten frei:n Tr rijodthyronin- ${ }^{125}$ Jods (2-5). Ein falsch hohes "freie: "Tr rijodthyronin- ${ }^{125}$ Jod findet sich bei Dysproteinämie, "unsıspezifischen Erkrankungen «, bei erhöhtem $\mathrm{CO}_{2}$-Patialdrdruck, unter Behandlung mit einer Reihe von Medkameienten (Sulfonamide, Salicylate, p-Aminosalicylsäure, ?heny ytoin, Dicumarin, Bromsulfalein) und bei genetisch oder ddurch Androgenbehandlung erniedrigtem Spiegel an thyrooxinbindendem Globulin. Falsch niedrige Werte des iogennannten freien Trijodthyronin- ${ }^{125}$ Jods werden bei erhöbhtem Spiegel an thyroxinbindendem Globulin (Gravicidität, Östrogen- oder Antiovulantienbehandlung, akate i intermittierende Porphyrie) beobachtet. Diese Störfakoren n führen insbesondere dann zu einem komplizierten lild, v wenn gleichzeitig eine Schilddrüsenfunktionsstörung im §Sinne der Über- oder Unterfunktion mit entsprechencen ÄÄnderungen der Schilddrüsenhormonspiegel im Blut rorlię̧gt.

Vorteile der $T_{3}$-in-vitro-Tests: Diese Methoden we'erden durch eine Jodverseuchung des Serums (Kontastmnittel, Medikamente) nicht beeinträchtigt, und die Patienten 1 werden nicht mit Radiojod belastet (wichtig für de Kirinderklinik). Bei Kombination des $\mathrm{T}_{3}$-in-vitro-Tests $\mathrm{mt}$ anderen Methoden der Schilddrüsenfunktionsdiagnostix $\left(\mathrm{PPB}^{127} \mathrm{I}-\right.$ Bestimmung, Radiojodtest) läßt sich eine Reile de.er erwähnten Störfaktoren der Schilddrüsenfunktionsdiaagnostik klären und die richtige Diagnose des Schlddriüüsenfunktionszustandes stellen (2-5).

\section{Literatur}

(1) Hamolsky, M. W., A. Golodetz. A. S. Freedberg: The plasma protein thyroid hormone complex in man. III. Further studies on the use of the in

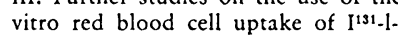
triiodo-thyronine as a diagnostic test of thyroid function. J. clin. Endocr. 19 (1959), 103.

(2) Oberdisse, K., E. Klein: Die Krank heiten der Schilddrüse (Stuttgart 1967).

(3) Schwarz, K., P. C. Scriba:

Privatdozent Dr. P. C. Scriba II. Medizinische Universitätsklinik 8 München 15 , Ziemssenstr. 1
Diagnostik und Therapie der Schchilddrüsenüberfunktion. Med Klin. 664 (1969), 469.

(4) Scriba, P. C., R. Lanigraf, H. G. Heinze, K. Schwarz Bestimmmung der Bindung von Trijodthronin a an Serumproteine mittels De:tran-GejelFiltration. Klin. Wschr. 4 (1966), ), 69. (5) Heinze, H. G., K. W. 'rey, P. C. Scriba: Methoden und Ergebəbnisse der Schilddrüsenfunktionsiagnostistik im bayerischen Jodmangelgebet. Fort rtschr. Röntgenstr. 108 (1968), 59 . 